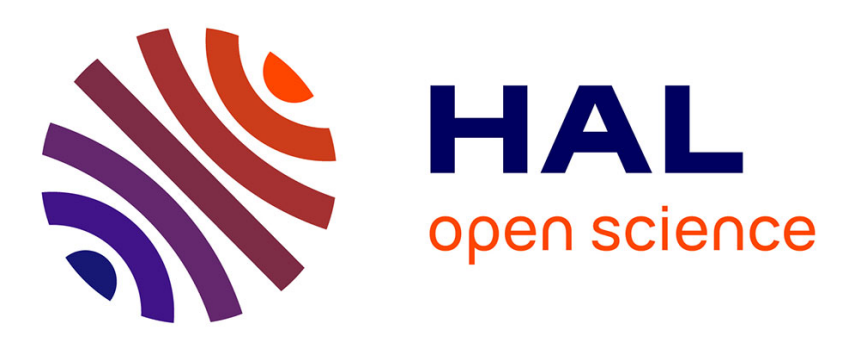

\title{
Fibrose rétropéritonéale: analyse descriptive rétrospective d'une cohorte de 56 patients
}

Inès Deghmani

\section{To cite this version:}

Inès Deghmani. Fibrose rétropéritonéale: analyse descriptive rétrospective d'une cohorte de 56 patients. Médecine humaine et pathologie. 2021. dumas-03395985

\section{HAL Id: dumas-03395985 https://dumas.ccsd.cnrs.fr/dumas-03395985}

Submitted on 22 Oct 2021

HAL is a multi-disciplinary open access archive for the deposit and dissemination of scientific research documents, whether they are published or not. The documents may come from teaching and research institutions in France or abroad, or from public or private research centers.
L'archive ouverte pluridisciplinaire $\mathbf{H A L}$, est destinée au dépôt et à la diffusion de documents scientifiques de niveau recherche, publiés ou non, émanant des établissements d'enseignement et de recherche français ou étrangers, des laboratoires publics ou privés. 


\title{
THÈSE POUR LE DOCTORAT EN MÉDECINE
}

(Diplômé d'État)

\author{
Par \\ Inès DEGHMANI \\ Née le 28 Janvier 1993, à Mont-Saint-Aignan \\ PRÉSENTÉE ET SOUTENUE PUBLIQUEMENT
}

LE 11 OCTOBRE 2021

\section{FIBROSE RÉTROPÉRITONÉALE : ANALYSE DESCRIPTIVE RÉTROSPECTIVE D'UNE COHORTE DE 56 PATIENTS}

PRÉSIDENT DU JURY : Monsieur le Professeur Hervé LEVESQUE

DIRECTEUR DE THÈSE : Monsieur le Docteur Guillaume ARMENGOL MEMBRES DU JURY : $\quad$ Monsieur le Professeur Dominique GUERROT Monsieur le Docteur Jean-Pierre LESTRAT Monsieur le Docteur Sébastien MIRANDA Monsieur le Docteur François-Xavier NOUHAUD 
DOYEN :

ASSESSEURS :
Professeur Benoît VEBER

Professeur Loïc FAVENNEC

Professeur Agnès LIARD

Professeur Guillaume SAVOYE

\section{I - MEDECINE}

\begin{tabular}{lll}
\multicolumn{2}{l}{ PROFESSEURS DES UNIVERSITES - PRATICIENS HOSPITALIERS } \\
\hline Mr Frédéric ANSELME & HCN & Cardiologie \\
Mme Gisèle APTER & Havre & Pédopsychiatrie \\
Mme Isabelle AUQUIT AUCKBUR & HCN & Chirurgie plastique \\
Mr Jean-Marc BASTE & HCN & Chirurgie Thoracique \\
Mr Fabrice BAUER & HCN & Cardiologie \\
Mme Soumeya BEKRI & HCN & Biochimie et biologie moléculaire \\
Mr Ygal BENHAMOU & HCN & Médecine interne \\
Mr Jacques BENICHOU & HCN & Bio statistiques et informatique médicale \\
Mr Olivier BOYER & UFR & Immunologie \\
Mme Sophie CANDON & HCN & Immunologie \\
Mr François CARON & HCN & Maladies infectieuses et tropicales \\
Mr Philippe CHASSAGNE & HCN & Médecine interne (gériatrie) \\
Mr Moïse COEFFIER & HCN & Nutrition \\
Mr Vincent COMPERE & HCN & Anesthésiologie et réanimation chirurgicale \\
Mr Jean-Nicolas CORNU & HCN & Urologie \\
Mr Antoine CUVELIER & HB & Pneumologie \\
Mr Jean-Nicolas DACHER & HCN & Radiologie et imagerie médicale \\
Mr Stéfan DARMONI & HCN & Informatique médicale et techniques de communication \\
Mr Pierre DECHELOTTE & HCN & Nutrition \\
Mr Stéphane DERREY & HCN & Neurochirurgie \\
Mr Frédéric DI FIORE & CHB & Cancérologie \\
& & \\
\hline & &
\end{tabular}




\author{
Mr Fabien DOGUET \\ Mr Jean DOUCET \\ Mr Bernard DUBRAY \\ Mr Frank DUJARDIN \\ Mr Fabrice DUPARC \\ Mr Éric DURAND \\ Mr Bertrand DUREUIL \\ Mme Hélène ELTCHANINOFF \\ Mr Manuel ETIENNE \\ Mr Thierry FREBOURG \\ Mr Pierre FREGER (surnombre) \\ Mr Jean François GEHANNO \\ Mr Emmanuel GERARDIN \\ Mme Priscille GERARDIN \\ M. Guillaume GOURCEROL \\ Mr Dominique GUERROT \\ Mme Julie GUEUDRY \\ Mr Olivier GUILLIN \\ Mr Claude HOUDAYER \\ Mr Fabrice JARDIN \\ Mr Luc-Marie JOLY \\ Mr Pascal JOLY \\ Mme Bouchra LAMIA \\ Mme Annie LAQUERRIERE \\ Mr Vincent LAUDENBACH \\ Mr Hervé LEFEBVRE \\ Mr Thierry LEQUERRE \\ Mme Anne-Marie LEROI \\ Mr Hervé LEVESQUE \\ Mme Agnès LIARD-ZMUDA \\ Mr Pierre Yves LITZLER \\ M. David MALTETE \\ Mr Christophe MARGUET \\ Mme Isabelle MARIE
}

HCN Chirurgie Cardio Vasculaire

SJ Thérapeutique - Médecine interne et gériatrie

CHB Radiothérapie

HCN Chirurgie orthopédique - Traumatologique

$\mathrm{HCN}$ Anatomie - Chirurgie orthopédique et traumatologique

HCN Cardiologie

$\mathrm{HCN}$ Anesthésiologie et réanimation chirurgicale

HCN Cardiologie

HCN Maladies infectieuses et tropicales

UFR Génétique

$\mathrm{HCN}$ Anatomie - Neurochirurgie

$\mathrm{HCN}$ Médecine et santé au travail

HCN Imagerie médicale

HCN Pédopsychiatrie

$\mathrm{HCN}$ Physiologie

HCN Néphrologie

$\mathrm{HCN}$ Ophtalmologie

HCN Psychiatrie Adultes

HCN Génétique

CHB Hématologie

$\mathrm{HCN}$ Médecine d'urgence

HCN Dermato - Vénéréologie

Havre Pneumologie

$\mathrm{HCN}$ Anatomie et cytologie pathologiques

$\mathrm{HCN}$ Anesthésie et réanimation chirurgicale

HB Endocrinologie et maladies métaboliques

HCN Rhumatologie

HCN Physiologie

$\mathrm{HCN}$ Médecine interne

$\mathrm{HCN}$ Chirurgie Infantile

HCN Chirurgie cardiaque

HCN Neurologie

$\mathrm{HCN}$ Pédiatrie

$\mathrm{HCN}$ Médecine interne 


\begin{tabular}{|c|c|c|}
\hline Mr Jean-Paul MARIE & $\mathrm{HCN}$ & Oto-rhino-laryngologie \\
\hline Mr LoÏc MARPEAU & $\mathrm{HCN}$ & Gynécologie - Obstétrique \\
\hline Mr Stéphane MARRET & $\mathrm{HCN}$ & Pédiatrie \\
\hline Mme Véronique MERLE & $\mathrm{HCN}$ & Épidémiologie \\
\hline Mr Pierre MICHEL & $\mathrm{HCN}$ & Hépato-gastro-entérologie \\
\hline M. Benoit MISSET (détachement) & $\mathrm{HCN}$ & Réanimation Médicale \\
\hline Mr Marc MURAINE & $\mathrm{HCN}$ & Ophtalmologie \\
\hline Mr Christian PFISTER & $\mathrm{HCN}$ & Urologie \\
\hline Mr Jean-Christophe PLANTIER & $\mathrm{HCN}$ & Bactériologie - Virologie \\
\hline Mr Didier PLISSONNIER & $\mathrm{HCN}$ & Chirurgie vasculaire \\
\hline Mr Gaëtan PREVOST & $\mathrm{HCN}$ & Endocrinologie \\
\hline \multicolumn{2}{|c|}{ Mr Jean-Christophe RICHARD (détachement) } & HCN Réanimation médicale - Médecine d'urgence \\
\hline Mr Vincent RICHARD & UFR & Pharmacologie \\
\hline Mme Nathalie RIVES & $\mathrm{HCN}$ & Biologie du développement et de la reproduction \\
\hline Mr Horace ROMAN (détachement) & $\mathrm{HCN}$ & Gynécologie - Obstétrique \\
\hline Mr Jean-Christophe SABOURIN & $\mathrm{HCN}$ & Anatomie - Pathologie \\
\hline Mr Mathieu SALAUN & $\mathrm{HCN}$ & Pneumologie \\
\hline Mr Guillaume SAVOYE & $\mathrm{HCN}$ & Hépato-gastrologie \\
\hline Mme Céline SAVOYE-COLLET & $\mathrm{HCN}$ & Imagerie médicale \\
\hline Mme Pascale SCHNEIDER & $\mathrm{HCN}$ & Pédiatrie \\
\hline Mr Lilian SCHWARZ & $\mathrm{HCN}$ & Chirurgie Viscérale et Digestive \\
\hline Mr Michel SCOTTE & $\mathrm{HCN}$ & Chirurgie digestive \\
\hline Mme Fabienne TAMION & $\mathrm{HCN}$ & Thérapeutique \\
\hline Mr Luc THIBERVILLE & $\mathrm{HCN}$ & Pneumologie \\
\hline Mr Hervé TILLY (surnombre) & $\mathrm{CHB}$ & Hématologie et transfusion \\
\hline M. Gilles TOURNEL & $\mathrm{HCN}$ & Médecine Légale \\
\hline Mr Olivier TROST & $\mathrm{HCN}$ & Anatomie -Chirurgie Maxillo-Faciale \\
\hline Mr Jean-Jacques TUECH & $\mathrm{HCN}$ & Chirurgie digestive \\
\hline Mr Benoît VEBER & $\mathrm{HCN}$ & Anesthésiologie - Réanimation chirurgicale \\
\hline Mr Pierre VERA & $\mathrm{CHB}$ & Biophysique et traitement de l'image \\
\hline Mr Éric VERIN & \multicolumn{2}{|r|}{ Médecine Physique et de Réadaptation } \\
\hline Mr Éric VERSPYCK & $\mathrm{HCN}$ & Gynécologie obstétrique \\
\hline Mr Olivier VITTECOQ & $\mathrm{HC}$ & Rhumatologie \\
\hline Mr David WALLON & $\mathrm{HCN}$ & Neurologie \\
\hline
\end{tabular}


MAITRES DE CONFERENCES DES UNIVERSITES - PRATICIENS HOSPITALIERS

\begin{tabular}{|c|c|c|}
\hline Mme Najate ACHAMRAH & $\mathrm{HCN}$ & Nutrition \\
\hline Mme Elodie ALESSANDRI-GRADT & $\mathrm{HCN}$ & Virologie \\
\hline Mme Noëlle BARBIER-FREBOURG & $\mathrm{HCN}$ & Bactériologie - Virologie \\
\hline Mr Emmanuel BESNIER & $\mathrm{HCN}$ & Anesthésiologie - Réanimation \\
\hline Mme Carole BRASSE LAGNEL & $\mathrm{HCN}$ & Biochimie \\
\hline Mme Valérie BRIDOUX HUYBRECHTS & $\mathrm{HCN}$ & Chirurgie Vasculaire \\
\hline Mr Gérard BUCHONNET & $\mathrm{HCN}$ & Hématologie \\
\hline Mme Mireille CASTANET & $\mathrm{HCN}$ & Pédiatrie \\
\hline Mme Nathalie CHASTAN & $\mathrm{HCN}$ & Neurophysiologie \\
\hline M. Vianney GILARD & $\mathrm{HCN}$ & Neurochirurgie \\
\hline Mr Serge JACQUOT & UFR & Immunologie \\
\hline Mr Joël LADNER & $\mathrm{HCN}$ & Épidémiologie, économie de la santé \\
\hline Mr Jean-Baptiste LATOUCHE & UFR & Biologie cellulaire \\
\hline M. Florent MARGUET & $\mathrm{HCN}$ & Histologie \\
\hline Mme Chloé MELCHIOR & $\mathrm{HCN}$ & Gastroentérologie \\
\hline M. Sébastien MIRANDA & $\mathrm{HCN}$ & Médecine Vasculaire \\
\hline Mr Thomas MOUREZ (détachement) & $\mathrm{HCN}$ & Virologie \\
\hline Mr Gaël NICOLAS & UFR & Génétique \\
\hline Mme Muriel QUILLARD & $\mathrm{HCN}$ & Biochimie et biologie moléculaire \\
\hline Mme Laëtitia ROLLIN & $\mathrm{HCN}$ & Médecine du Travail \\
\hline Mme Pascale SAUGIER-VEBER & $\mathrm{HCN}$ & Génétique \\
\hline M. Abdellah TEBANI & $\mathrm{HCN}$ & Biochimie et Biologie Moléculaire \\
\hline Mme Anne-Claire TOBENAS-DUJARDIN & $\mathrm{HCN}$ & Anatomie \\
\hline Mr Julien WILS & $\mathrm{HCN}$ & Pharmacologie \\
\hline
\end{tabular}

\section{PROFESSEUR AGREGE OU CERTIFIE}

$\begin{array}{lll}\text { Mr Thierry WABLE } & \text { UFR } & \text { Communication } \\ \text { Mme Mélanie AUVRAY-HAMEL } & \text { UFR } & \text { Anglais }\end{array}$

\section{ATTACHE TEMPORAIRES D'ENSEIGNEMENT ET DE RECHERCHE à MI-TEMPS}




\section{PROFESSEURS DES UNIVERSITES}

Mr Jérémy BELLIEN (PU-PH)

Mr Thierry BESSON

Mr Jean COSTENTIN (Professeur émérite)

Mme Isabelle DUBUS

Mr Abdelhakim EL OMRI

Mr François ESTOUR

Mr Loïc FAVENNEC (PU-PH)

Mr Jean Pierre GOULLE (Professeur émérite)

Mme Christelle MONTEIL

Mme Martine PESTEL-CARON (PU-PH)

Mr Rémi VARIN (PU-PH)

Mr Jean-Marie VAUGEOIS

Mr Philippe VERITE

\section{MAITRES DE CONFERENCES DES UNIVERSITES}

Mme Cécile BARBOT

Mr Frédéric BOUNOURE

Mr Thomas CASTANHEIRO MATIAS

Mr Abdeslam CHAGRAOUI

Mme Camille CHARBONNIER (LE CLEZIO)

Mme Elizabeth CHOSSON

Mme Marie Catherine CONCE-CHEMTOB

Mme Cécile CORBIERE

Mme Nathalie DOURMAP

Mme Isabelle DUBUC

Mme Dominique DUTERTE- BOUCHER

Mr Gilles GARGALA (MCU-PH)
Pharmacologie

Chimie Thérapeutique

Pharmacologie

Biochimie

Pharmacognosie

Chimie Organique

Parasitologie

Toxicologie

Toxicologie

Microbiologie

Pharmacie clinique

Pharmacologie

Chimie analytique
Chimie Générale et Minérale

Pharmacie Galénique

Chimie Organique

Physiologie

Statistiques

Botanique

Législation pharmaceutique et économie de la santé

Biochimie

Pharmacologie

Pharmacologie

Pharmacologie

Parasitologie 
Mme Nejla EL GHARBI-HAMZA

Mme Marie-Laure GROULT

Mr Chervin HASSEL

Mme Maryline LECOINTRE

Mme Hong LU

Mme Marine MALLETER

M. Jérémie MARTINET (MCU-PH)

M. Romy RAZAKANDRAINIBÉ

Mme Tiphaine ROGEZ-FLORENT

Mr Mohamed SKIBA

Mme Malika SKIBA

Mme Christine THARASSE

Mr Frédéric ZIEGLER

\section{PROFESSEURS ASSOCIES}

Mme Cécile GUERARD-DETUNCQ

Mme Caroline BERTOUX

\section{PAU-PH}

M. Mikaël DAOUPHARS

\section{PROFESSEUR CERTIFIE}

Mme Mathilde GUERIN

\section{ASSISTANTS HOSPITALO-UNIVERSITAIRES}

Mme Alice MOISAN

M. Henri GONDÉ
Chimie analytique

Botanique

Biochimie et Biologie Moléculaire

Physiologie

Biologie

Toxicologie

Immunologie

Parasitologie

Chimie analytique

Pharmacie galénique

Pharmacie galénique

Chimie thérapeutique

Biochimie

Pharmacie officinale

Pharmacie

Anglais

\section{ATTACHES TEMPORAIRES D'ENSEIGNEMENT ET DE RECHERCHE}

Mme Soukaina GUAOUA-ELJADDI

Mme Clémence MEAUSOONE

\section{ATTACHE TEMPORAIRE D'ENSEIGNEMENT}

Mme Ramla SALHI
Informatique

Toxicologie

Pharmacognosie 
Mme Cécile BARBOT

Mr Thierry BESSON

Mr Abdeslam CHAGRAOUI

Mme Élisabeth CHOSSON

Mme Marie-Catherine CONCE-CHEMTOB

Mme Isabelle DUBUS

Mr Abdelhakim EL OMRI

Mr François ESTOUR

Mr Loïc FAVENNEC

Mr Michel GUERBET

Mme Martine PESTEL-CARON

Mr Mohamed SKIBA

Mr Rémi VARIN

M. Jean-Marie VAUGEOIS

Mr Philippe VERITE
Chimie Générale et minérale

Chimie thérapeutique

Physiologie

Botanique

Législation et économie de la santé

Biochimie

Pharmacognosie

Chimie organique

Parasitologie

Toxicologie

Microbiologie

Pharmacie galénique

Pharmacie clinique

Pharmacologie

Chimie analytique 


\section{PROFESSEUR MEDECINE GENERALE}

Mr Jean-Loup HERMIL (PU-MG)

UFR Médecine générale

MAITRE DE CONFERENCE MEDECINE GENERALE

Mr Matthieu SCHUERS (MCU-MG)

UFR Médecine générale

PROFESSEURS ASSOCIES A MI-TEMPS - MEDECINS GENERALISTE

Mr Pascal BOULET

UFR Médecine générale

Mr Emmanuel LEFEBVRE

UFR Médecine Générale

Mme Élisabeth MAUVIARD

UFR Médecine générale

Mr Philippe NGUYEN THANH

UFR Médecine générale

Mme Yveline SEVRIN

UFR Médecine générale

\section{MAITRE DE CONFERENCES ASSOCIE A MI-TEMPS - MEDECINS GENERALISTES}

Mme Laëtitia BOURDON

Mme Elsa FAGOT-GRIFFIN

Mr Emmanuel HAZARD

Mme Lucile PELLERIN

\author{
UFR Médecine Générale \\ UFR Médecine Générale \\ UFR Médecine Générale \\ UFR Médecine générale
}




\section{PROFESSEURS}

Mr Paul MULDER (phar)

Sciences du Médicament

Mme Su RUAN (med)

Génie Informatique

\section{MAITRES DE CONFERENCES}

Mr Sahil ADRIOUCH (med)

Biochimie et biologie moléculaire (Unité Inserm 905)

Mme Gaëlle BOUGEARD-DENOYELLE (med)

Biochimie et biologie moléculaire (UMR 1079)

Mme Carine CLEREN (med)

Neurosciences (Néovasc)

M. Sylvain FRAINEAU (med)

Physiologie (Inserm U 1096)

Mme Pascaline GAILDRAT (med)

Génétique moléculaire humaine (UMR 1079)

Mr Nicolas GUEROUT (med)

Chirurgie Expérimentale

Mme Rachel LETELLIER (med)

Physiologie

Mr Antoine OUVRARD-PASCAUD (med)

Physiologie (Unité Inserm 1076)

Mr Frédéric PASQUET

Sciences du langage, orthophonie

Mme Anne-Sophie PEZZINO

Orthophonie

Mme Christine RONDANINO (med)

Physiologie de la reproduction

Mr Youssan Var TAN

Immunologie

Mme Isabelle TOURNIER (med)

Biochimie (UMR 1079)

DIRECTEUR ADMINISTRATIF : M. Jean-Sébastien VALET 
Par délibération en date du 3 mars 1967, la faculté a arrêté que les opinions émises dans les dissertations qui lui seront présentées doivent être considérées comme propres à leurs auteurs et qu'elle n'entend leur donner aucune approbation ni improbation. 


\section{LISTE DES ABRÉVIATIONS}

AAA : Anévrysme de l'Aorte Abdominale

AAN : Anticorps Antinucléaire

ADC : Apparent Diffusion Coefficent

ANCA : Anti-Neutrophil Cytoplasmic Antibody

$\mathrm{CCL}: \mathrm{C}-\mathrm{C}$ motif chemokine Ligand

CCR5 : Cysteine-Cysteine chemokine Receptor type 5

CD : Cluster de Différenciation

CFG : Champ à Fort Grossissement

CRP : Protéine C Réactive

CT : Computed Tomography

FDG : FluoroDéoxyGlucose

FDRCV : Facteur de Risque Cardio-Vasculaire

FRP : Fibrose RétroPéritonéale

FRPi : Fibrose RétroPéritonéale Idiopathique

HLA : Human Leucocyte Antigen

LDL : Low Density Lipoprotein

Ig : ImmunoGlobuline

IL : InterLeukine

IRA : Insuffisance Rénale Aigue

IRM : Imagerie par Résonnance Magnétique

IRC : Insuffisance Rénale Chronique

MAG4 : Maladie Associée aux IgG4

Mg: MilliGrammes

MIP : Macrophage Inflammatory Protein

PAN : PériArtérite Noueuse 
PET : Positron Emission Tomography

PC : Péri-aortite Chronique

RANTES : Regulated upon Activation Normal T cell Expressed and Secreted

TDM : TomoDensitoMétrie

Th : T helper

VS : Vitesse de Sédimentation des érythrocytes 


\section{TABLE DES MATIÈRES}

$\begin{array}{ll}\text { I. Introduction } & 20\end{array}$

$\begin{array}{ll}\text { A. Généralités } & 20\end{array}$

B. Épidémiologie 20

C. Nosologie 21

D. Étiologies et facteurs de risque $\quad 22$

1. Fibrose rétropéritonéale idiopathique 22

a. Étiologies 22

b. Facteurs environnementaux 22

c. Facteurs génétiques 22

d. Association aux maladies auto immunes 23

2. Fibroses rétropéritonéales secondaires 23

3. Maladie associée aux IgG4 24

a. Généralités 24

b. Histologie 25

$\begin{array}{ll}\text { C. Diagnostic } 26 & 26\end{array}$

d. Fibrose rétropéritonéale de la maladie associée aux IgG4 27

$\begin{array}{ll}\text { E. Physiopathologie } & 28\end{array}$

1. Hypothèses physiopathologiques 28

2. Mécanisme immunopathologique 29

F. Manifestations cliniques 30

1. Complications urologiques 31

2. Complications vasculaires 31

3. Complications génitales et abdominales 31

G. Examens biologiques 32

H. Histologie 32

1. Fibrose rétropéritonéale idiopathique 32

2. Fibrose rétropéritonéale secondaire 34 
$\begin{array}{ll}\text { I. Imagerie } 34 & 34\end{array}$

1. Échographie 34

2. Tomodensitométrie 34

3. Imagerie par résonnance magnétique 36

$\begin{array}{ll}\text { 4. PET TDM } & 37\end{array}$

5. Diagnostics différentiels en imagerie 38

J. Diagnostic de fibrose rétropéritonéale idiopathique 39

$\begin{array}{ll}\text { K. Traitement } & 40\end{array}$

1. Prise en charge urologique 40

2. Prise en charge médicale des FRPi 41

$\begin{array}{ll}\text { a. Corticothérapie } & 41\end{array}$

b. Tamoxifène 42

c. Traitements de seconde ligne 42

3. Prise en charge de la fibrose rétropéritonéale secondaire 44

L. Évolution et suivi $\quad 44$

II. Objectif de l'étude $\quad 45$

III. Patients et méthode 46

A. Inclusion des patients $\quad 46$

B. Diagnostic de fibrose rétropéritonéale $\quad 46$

C. Recueil de données $\quad 47$

D. Analyses statistiques $\quad 49$

$\begin{array}{ll}\text { IV. Résultats } & 50\end{array}$

A. Caractéristiques de la population 51

1. Population globale 51

2. Fibroses rétropéritonéales idiopathiques et secondaires 51

B. Étiologies 53

C. Présentation clinique $\quad 55$

D. Présentation biologique 56

E. Présentation radiologique $\quad 57$

F. Prise en charge initiale $\quad 58$ 
G. Rechutes $\quad 59$

$\begin{array}{ll}\text { H. Récidives } & 60\end{array}$

$\begin{array}{ll}\text { I. Évolution } & 61\end{array}$

J. Population AAA et ectasie de l'aorte 63

1. Caractéristiques globales 63

2. Données cliniques, biologiques, radiologiques et histologiques 64

3. Prise en charge 66

4. Rechutes et récidives 66

5. Évolution 68

$\begin{array}{ll}\text { V. Discussion } & 69\end{array}$

A. Critères d'inclusion des patients $\quad 69$

B. Épidémiologie 69

$\begin{array}{ll}\text { C. Hypothèse de l'auto-immunité } & 70\end{array}$

$\begin{array}{ll}\text { D. FRP secondaires } & 71\end{array}$

E. Données cliniques, biologiques et histologiques 72

$\begin{array}{ll}\text { F. Imagerie } & 73\end{array}$

G. Prise en charge thérapeutique $\quad 75$

H. Évolution et suivi $\quad 76$

$\begin{array}{ll}\text { I. Limites du travail } & 77\end{array}$

$\begin{array}{ll}\text { VI. Conclusion } & 78\end{array}$

$\begin{array}{ll}\text { VII. Références bibliographiques } & 79\end{array}$ 


\section{Introduction}

\section{A. Généralités}

La fibrose rétropéritonéale idiopathique (FRPi) est une maladie rare caractérisée par la présence d'un tissu fibro-inflammatoire aberrant se formant dans l'espace rétropéritonéal et engainant les structures de continuité (aorte, artères iliaques, veine cave, uretères). Elle provoque fréquemment une uropathie obstructive et une insuffisance rénale.

Le premier cas fut rapporté en 1905 par l'urologue français Albarran, avec la description d'une hydronéphrose secondaire à une fibrose rétropéritonéale (FRP) extensive.

C'est en 1948 que la maladie devient une entité clinique avec la description de deux cas par Ormond, conférant initialement le nom de "maladie de Ormond " à cette atteinte. ${ }^{1}$ Le terme de fibrose rétropéritonéale est introduit pour le première fois par Raper en $1956 .^{2}$

Depuis, la compréhension de la physiopathologie, les progrès de l'imagerie mais également les avancées chirurgicales ont permis une amélioration de la prise en charge de la FRPi. A ce jour, plus de 2000 cas ont été décrits dans la littérature.

L'évolution dans la compréhension de cette pathologie voit désormais apparaître le terme de péri-aortite chronique (PC), englobant la fibrose rétropéritonéale idiopathique, les anévrysmes de l'aorte abdominale (AAA) inflammatoires et les anévrysmes de l'aorte avec fibrose péri-anévrysmale.

Le traitement de la FRPi vise à éviter ou faire diminuer l'obstruction urétérale et à faire régresser l'inflammation liée à la maladie. Le traitement de référence est la corticothérapie, utilisée en monothérapie ou en association avec d'autres thérapeutiques.

\section{B. Épidémiologie}

La FRPi est une maladie rare dont l'incidence et la prévalence précises sont mal connues. Dans une étude finlandaise cas-témoins, l'incidence a été évaluée à 0,1 pour 100000 habitants et la prévalence à 1,3/100 000 habitants ${ }^{3}$. Plus récemment, une étude hollandaise, prospective, monocentrique, portant sur une période de 10 ans a évalué l'incidence à 1,3/100 000 habitants ${ }^{4}$.

Alors que les maladies auto-immunes touchent préférentiellement les femmes jeunes, la FRPi est plus fréquente chez les hommes avec un sexe-ratio allant de 2 à 3 hommes pour 1 femme selon les séries ${ }^{5}$. L'âge moyen au diagnostic varie de 50 à 60 ans, bien que d'exceptionnelles formes pédiatriques soient décrites ${ }^{6}$. 


\section{Nosologie}

La FRPi s'inscrit désormais dans un groupe plus vaste appelé péri-aortites chroniques (PC) comprenant les anévrysmes inflammatoires de l'aorte, les FRP péri-anévrysmales et les FRP idiopathiques. (Figure 1)

Ces trois entités ont des caractéristiques histo-pathologiques communes : inflammation adventitielle et péri adventitielle, amincissement et athérosclérose médiale.

Dans le cas de la FRPi, l'aorte n'est pas dilatée et le tissu fibro-inflammatoire qui l'entoure peut envelopper les structures adjacentes.

Dans le cas des anévrysmes aortiques inflammatoires, le tissu se développe autour d'une aorte dilatée mais ne provoque pas l'obstruction des structures adjacentes.

Enfin la fibrose péri-anévrysmale est un anévrysme inflammatoire qui est au contact des structures adjacentes $5,7,8$.

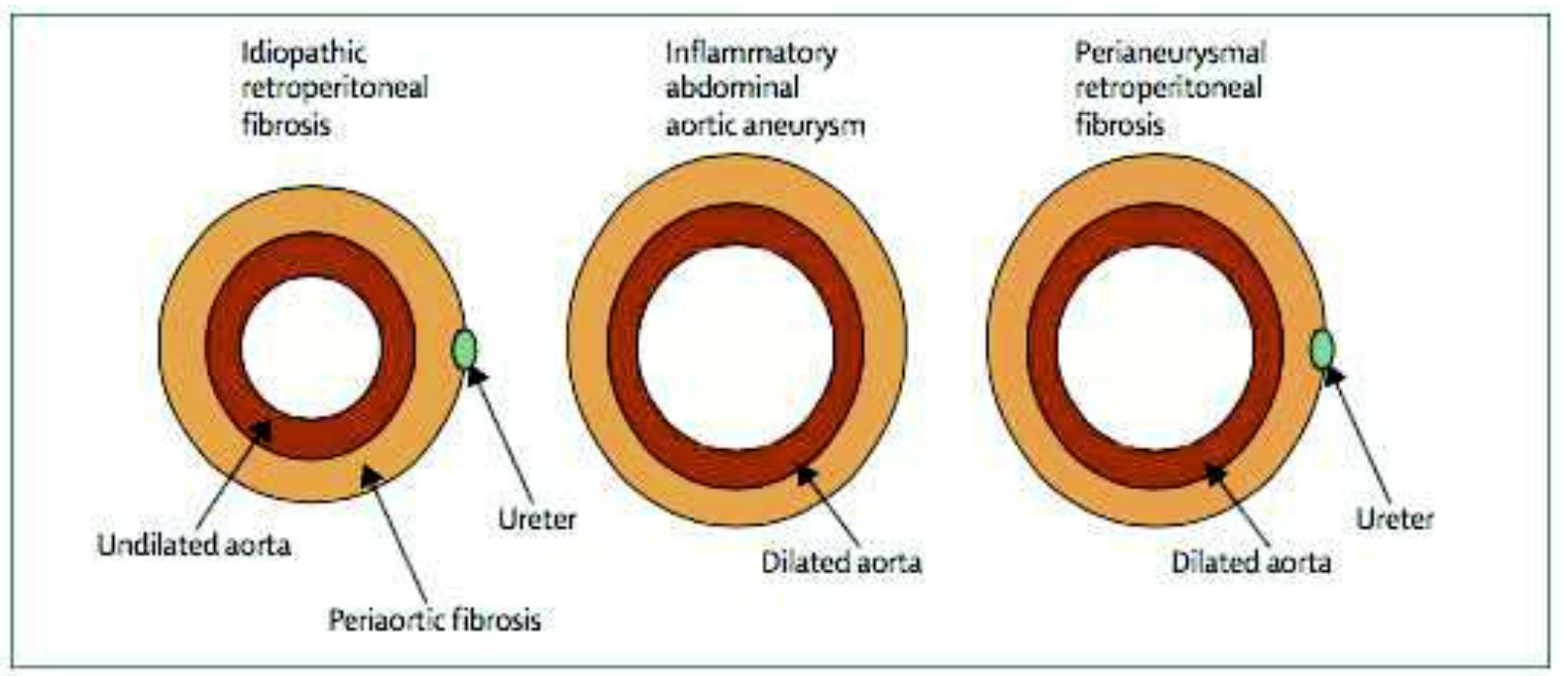

Figure 1. Représentation schématique des péri-aortites chroniques.

De gauche à droite : fibrose rétropéritonéale, anévrysme aortique inflammatoire, fibrose péri-anévrysmale (D’après Vaglio A et al. Lancet. 2006) 


\section{D. Étiologies et facteurs de risque}

\section{Fibrose rétropéritonéale idiopathique}

\section{a. Étiologies}

Lorsqu'aucune cause n'est retrouvée, la fibrose rétropéritonéale est dite « idiopathique ».

Il existe un lien fort entre FRPi et facteurs de risques cardiovasculaires (FDRCV), et il a longtemps été admis que les péri-aortites étaient le résultat d'une inflammation locale en réponse à des antigènes contenus dans les plaques d'athérome, comme les lipoprotéines de faible densité (LDL) oxydés.

Néanmoins, certains patients ayant une FRPi n'ont pas d'athérome visible à l'imagerie ni FDRCV. Le résultat des études menées au cours des dix dernières années soutiennent plutôt l'existence d'une maladie systémique sous-jacente ${ }^{9,10}$. Les observations cliniques telles que la présence de symptômes systémiques, l'association avec d'autres manifestations auto-immunes ainsi que la bonne réponse aux traitements immunosuppresseurs renforcent cette hypothèse. Par ailleurs, il a été rapporté que la FRPi affecte parfois d'autres segments vasculaires (par exemple, l'aorte thoracique, les artères mésentériques), généralement moins concernées par l'athérosclérose ${ }^{11,12}$.

\section{b. Facteurs environnementaux}

$\mathrm{L}^{\prime}$ amiante pourrait être un facteur de risque de $\mathrm{FRPi}^{3}$. Une étude italienne cas-témoins portant sur 90 patients atteints de FRPi et 270 témoins indemnes a confirmé le rôle prédisposant de l'exposition à l'amiante (OR, 4.22 [IC 95\% 2.14-8.33]), mais également du tabagisme actif (OR, 3.21 [IC 95\% 1.46-7.07]) et du tabagisme sevré (OR, 2.93 [IC 95\% 1.39-6.14]) ${ }^{13}$.

\section{c. Facteurs génétiques}

Les résultats d'une étude cas-témoins ${ }^{14}$ portant sur 35 patients ont montré que la FRPi était associée de manière significative au HLA-DRB1*03, un allèle lié à diverses maladies auto-immunes (diabète de type 1 , myasthénie auto-immune, thyroïdite auto-immune).

Une autre étude cas-témoins a également décrit une susceptibilité accrue à développer une FRP, en particulier dans sa forme anévrysmale, chez les patients porteurs du polymorphisme delta $32 \mathrm{du}$ gène du récepteur 5 des CC-chemokines (CCR5) ${ }^{15}$. Le CCR5 est exprimé sur de nombreuses cellules immunitaires, notamment les cellules Th1, et agit en se liant à plusieurs chimiokines (dont RANTES, MIP-1alpha et MIP- 
1beta). Le polymorphisme du CCR5delta32 créé un récepteur tronqué et non fonctionnel faisant probablement évoluer la réponse immunitaire vers un modèle Th2.

\section{d. Association aux maladies auto immunes}

La FRPi peut être associée à une grande variété de maladies auto-immunes, ce qui renforce la pertinence pathogénique des mécanismes auto-immuns.

La thyroïdite auto-immune est la plus fréquemment associée : dans une étude cas-témoins, les patients atteints de FRPi avaient une prévalence d'anticorps anti-thyroperoxydase de 24,7\% (contre 10,6\% chez les témoins sains) et des signes échographiques de thyroïdite. Après un suivi médian de 45 mois, $25 \%$ des patients atteints de FRP ont développé une hypothyroïdie. Lorsqu'elle était disponible, l'histologie a montré une thyroïdite de Hashimoto typique ou sa variante fibreuse ${ }^{16}$.

Parmi les autres associations, on peut citer la polyarthrite rhumatoïde ${ }^{17}$, la spondylarthrite ankylosante ${ }^{18}$, les vascularites associées aux ANCA ${ }^{19-21}$, le lupus érythémateux systémique ${ }^{22}$ et le psoriasis 23 .

D'autres maladies fibro-inflammatoires sont fréquemment associés à la FRPi : thyroïdite de Riedel ${ }^{24}$, pancréatite et cholangite sclérosantes, sialoadénite/dacryoadénite sclérosante, pseudotumeur orbitaire et médiastinite sclérosante. Ces maladies peuvent coexister et correspondent à ce que l'on appelait autrefois la fibrosclérose multifocale. On parle dorénavant de maladie associée aux IgG4 ${ }^{25,26}$.

\section{Fibroses rétropéritonéales secondaires}

De nombreuses causes de FRP secondaires ont été identifiées (tableau 1) : tumorale (lymphomes, sarcomes, métastases), médicamenteuses (dérivés de l'ergot de seigle, méthyldopa, bêtabloquants...), infectieuses (tuberculose, histoplasmose, actinomycose), post-radiothérapie, post chirurgicales, et d'autres plus rares encore (histiocytose, maladie d'Erdheim-Chester, amylose...).

La FRP tumorale est de mauvais pronostic. Elle peut être le résultat d'une réaction inflammatoire en réponse à la présence de cellules malignes métastatiques présentes dans le rétropéritoine. Ces cellules sont principalement secondaires au carcinome de la prostate, du sein, du colon et du cancer du col utérin. Elle peut aussi être secondaire à une tumeur maligne rétropéritonéale primitive (lymphome de Hodgkin ou non Hodgkinien, sarcomes ...) ${ }^{27,28}$.

En ce qui concerne les médicaments, les associations les plus fréquentes sont avec les dérivés de l'ergot de seigle ou avec les agonistes de la dopamine. Le méthysergide et les autres agents dérivés de 
l'ergotamine augmentent les niveaux de sérotonine endogène et il a été suggéré que cela entraînait des réactions fibrotiques par la prolifération de myofibroblastes et l'augmentation du dépôt de matrice collagène. Cet effet n'est pas seulement limité au rétropéritoine, mais peut également concerner le péricarde ou la plèvre ${ }^{29}$. D'autres médicaments rapportés comme étant associés à la FRPi sont les beta bloquants ou I'hydralazine mais ce lien de cause à effet est toujours débattu ${ }^{5,30}$. Récemment, des cas de FRPi ont été rapportés à l'utilisation d'infliximab ou d'etanercept ${ }^{31}$. Le lien physiopathologique n'est pas élucidé, mais ces traitements sont connus pour déclencher un certain nombre de manifestations auto-immunes.

\begin{tabular}{|l|l|}
\hline Médicaments & $\begin{array}{l}\text { Béta bloquants (propranolol, sotalol, oxyprenolol, atenolol), } \\
\text { methylsergide, bromocriptine, ergotamine, methyldopa, hydralazine, } \\
\text { infliximab }\end{array}$ \\
\hline Néoplasies & $\begin{array}{l}\text { Hémopathies (maladie de Hodgkin et lymphomes non Hodgkiniens) } \\
\text { Métastases (colon, poumons, uro-génitaux, sein, pancréas) } \\
\text { Tumeurs carcinoïdes } \\
\text { Sarcomes rétro-péritonéaux }\end{array}$ \\
\hline Infections & $\begin{array}{l}\text { Tuberculose } \\
\text { Histoplasmose } \\
\text { Actinomycose } \\
\text { Maladie d'Erdheim-Chester, amylose, post traumatique/chirurgie, } \\
\text { post radiothérapie }\end{array}$ \\
\hline Autres & \\
\hline
\end{tabular}

Tableau 1 : Principales étiologies de FRP secondaires. (D'après Vaglio A et al. Lancet. 2006)

\section{Maladie associée aux IgG4}

\section{a. Généralités}

Une proportion significative de patient ayant une FRPi pourrait correspondre à une maladie associée aux IgG4 (MAG4), entre 30 et $59 \%^{32}$.

La maladie associée aux IgG4 est de description récente. Elle se caractérise par la présence d'une ou plusieurs atteintes fibro-inflammatoires d'organes, associées le plus souvent à une élévation des IgG4 sériques $^{33}$. Cependant, ce résultat n'est ni sensible ni spécifique pour le diagnostic ou pour le suivi de l'activité de la maladie. Les lésions tissulaires sont caractérisées par une fibrose et un infiltrat inflammatoire lymphocytaire et plasmocytaire, avec prédominance de plasmocytes IgG4+.

Les atteintes rapportées sont nombreuses : pancréatite sclérosante, sialadénite, dacryoadénite, polyadénopathies, aortite, cholangite sclérosante, néphrite interstitielle, fibrose rétropéritonéale ou encore pseudotumeurs inflammatoires orbitaires. (figure 2) 

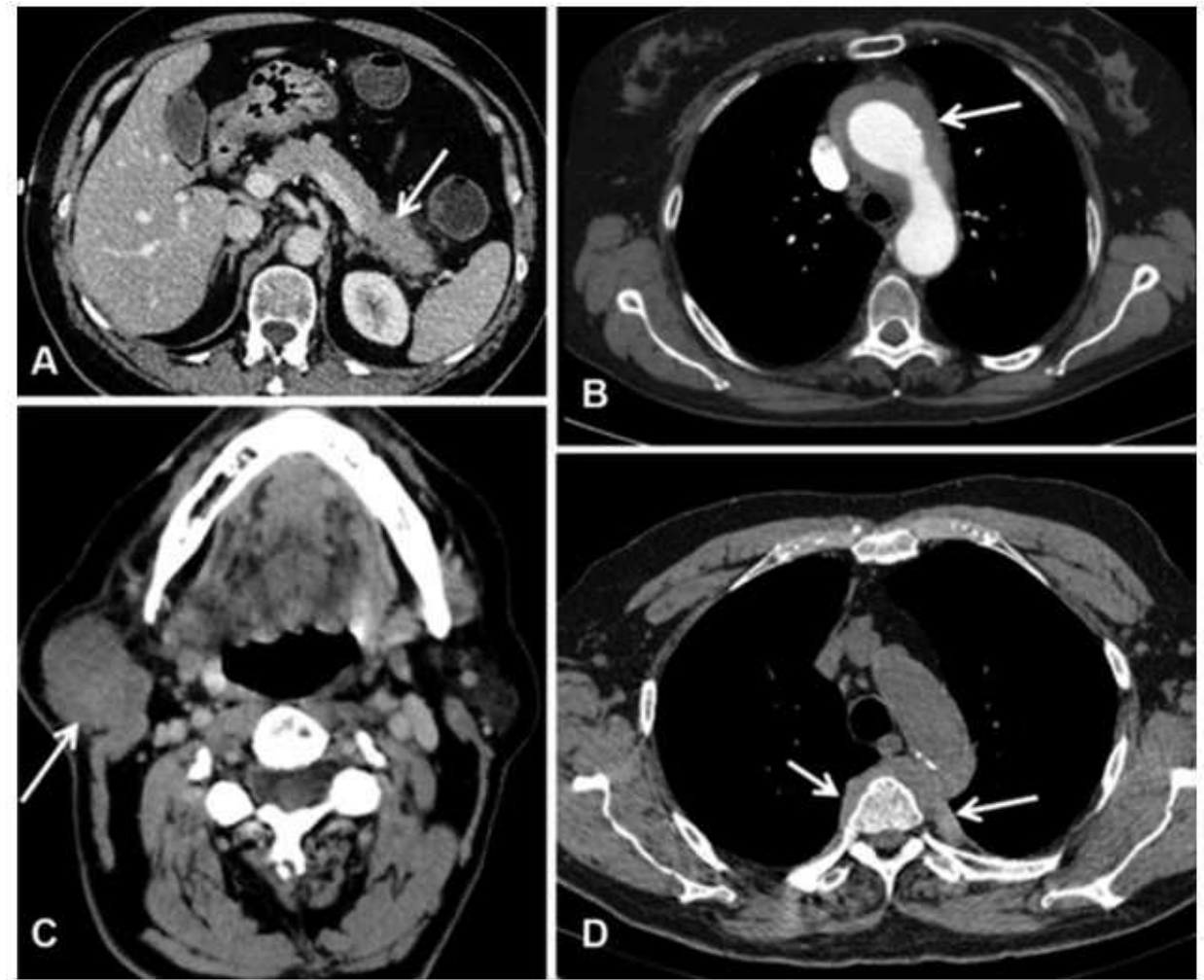

Figure 2 : Images courantes de tomodensitométries de maladies associées aux IgG4 (D'après Rossi et al. Internal and Emergency Medecine, 2017)
A : Pancréatite auto-immune de type 1 (hypertrophie de la queue du pancréas)
B : Péri aortite thoracique. C : Sialadénite chronique sclérosante de la glande parotide.
D : Médiastinite fibrosante

\section{b. Histologie}

Les principales anomalies histologiques observées sont l'existence d'un infiltrat lymphoplasmocytaire dense, d'une fibrose "storiforme " (enroulement des faisceaux fibreux réalisant un aspect en « roue de carrosse ») et la présence de « phlébites oblitérantes » (oblitération partielle ou complète des veines, qui apparaissent comme des nodules inflammatoires à côté d'une artère perméable). Un infiltrat éosinophile tissulaire modéré est également fréquemment observé. L'examen immunohistochimique permet le marquage et le compte des plasmocytes lgG4+ par champ à fort grossissement (CFG) ${ }^{33,34}$ (figure 3) 

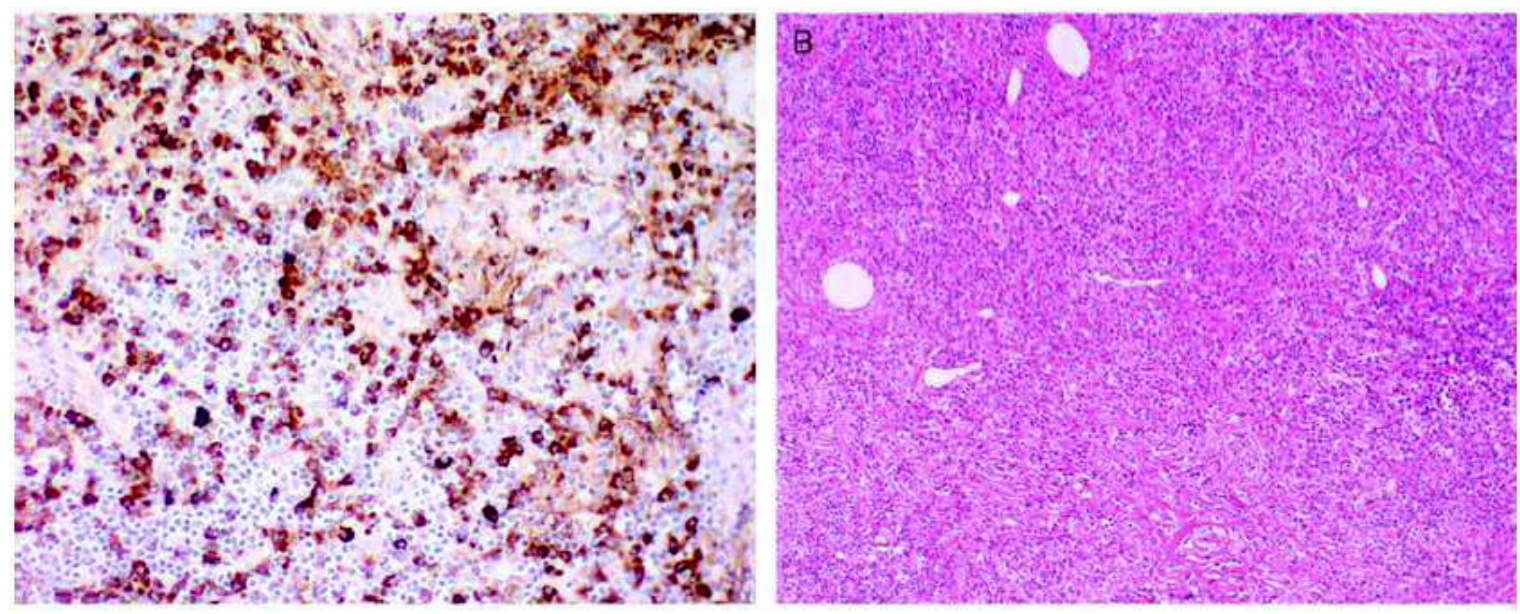

Figure 3 : Anomalies histologiques d'une FRP liée à une maladie des IgG4 (D'après Zen et al. American Journal of Surgical Pathology. 2009)

A : Immunomarquage plasmocytes IgG4+

B : Infiltration lymphoplasmocytaire et fibrose storiforme

\section{c. Diagnostic}

Les premiers critères diagnostiques reposaient sur des éléments cliniques, biologiques et histologiques (tableau 2), proposés par un consensus Japonais en $2011^{35,36}$.

\section{Tableau 2 Critères « CDC » (Comprehensive Diagnostic Criteria) de la maladie associée aux IgG4}

1. Clinique : Hypertrophie localisée ou diffuse au sein d'un ou plusieurs organes classiquement atteints au cours de la MAG4

2. Biologie : Elévation des $\lg G 4$ sériques $(>/=1,35 \mathrm{~g} / \mathrm{L})$

3. Histologie :

- Infiltration lymphocytaire et plasmocytaire polyclonale marquée + fibrose

- Infiltration par des plasmocytes IgG4+ : ratio cellules $\lg G 4+/ \lg G+>40 \%$ et $>10$ plasmocytes IgG4+/CFG

Des critères spécifiques d'atteintes d'organes ont été proposés par différents auteurs comme par exemple les critères diagnostiques spécifiques de la pancréatite auto immunes associées aux $\operatorname{lgG} 4{ }^{37}$. Des critères diagnostiques spécifiques de l'atteinte vasculaire péri-aortique, artérielle et rétro-péritonéale ont 
été également proposés par un groupe d'auteur japonais ${ }^{38}$, incluant des critères biologiques, histologiques et d'imagerie.

\section{d. Fibrose rétropéritonéale de la maladie associée aux IgG4}

Bien qu'il soit désormais admis que la FRP fasse partie du spectre des MAG4, la proportion de MAG4 parmi les FRPi reste inconnue. Par ailleurs, les différences cliniques, la variabilité de réponse au traitement et le pronostic entre FRPi et FRP liées aux IgG4 n'ont pas été étudiées.

Quelques études, principalement rétrospectives et sur de petits effectifs, ont abordé ces questions. La plupart des auteurs ont classé les FRP comme liées ou non aux IgG4 en se basant sur des critères histologiques et immunohistochimiques ${ }^{39-44}$. Le pourcentage de FRP liées aux IgG4 semble se situer entre 30 et $59 \%$. Dans la FRP liée aux IgG4, il y a une fréquence plus élevée de lésions extra-rétropéritonéales ${ }^{40,41}$. Certaines de ces études ont également montré une prédominance masculine dans le sous-groupe lié aux IgG4.

De façon intéressante, les deux groupes ne présentent pas de différence significative en termes de complications rétropéritonéales (atteinte urétérale) ou de caractéristiques tomodensitométriques de la fibrose (localisation, atteinte vasculaire, épaisseur).

Aucune de ces études n'a clairement évalué la réponse au traitement de la FRP liée aux IgG4 par rapport à la FRP non liée aux IgG4. Par conséquent, la prétendue plus grande cortico-sensibilité des formes liées aux IgG4 reste à prouver. Une seule étude a montré un taux de rechute plus élevé dans la FRP liée aux $\operatorname{lgG} 4{ }^{42}$.

Dans l'ensemble, ces deux formes de FRP représentent les deux extrémités d'un même spectre pathologique qui partagent potentiellement des mécanismes pathogéniques communs. La question de savoir s'ils répondent différemment aux traitements demeure non élucidée. Le développement de nouvelles alternatives thérapeutiques comme le rituximab pose de nouveaux questionnements. À I'heure actuelle, il n'existe pas de données solides étayant l'hypothèse selon laquelle seule la FRP liée aux IgG4 est sensible à ce type de traitement, puisque les formes liées et non liées aux $\lg G 4$ ont toutes été décrites comme présentant des réponses cliniques positives ${ }^{45-47}$. 


\section{E. Physiopathologie}

\section{Hypothèses physiopathologiques}

La physiopathologie de la FRPi n'est pas claire et l'étiologie est probablement multifactorielle. II est largement observé que la fibrose rétropéritonéale est le plus souvent associée à des lésions athéromateuses. Les péri-aortites chroniques pourraient être le résultat d'une réaction inflammatoire locale exagérée aux LDL oxydés et aux céroïdes, souvent présents dans les plaques d'athérome de l'aorte abdominale (figure 4). Il est suggéré que lorsque la média de l'aorte est altérée, ces lipides oxydés sont présentés par les macrophages de la plaque aux cellules immunocompétentes telles que les lymphocytes T et $B$. Ces derniers sont recrutés et activés dans la média et l'adventice de l'aorte. Cette action initie une réponse inflammatoire autoentretenue qui conduit finalement à une inflammation de la paroi aortique principalement localisée dans la média et l'adventice, et qui s'étend ensuite dans le rétropéritoine péri-aortique. ${ }^{5,8}$

A l'inverse, la FRPi pourrait au contraire être la manifestation d'une maladie auto-immune systémique et se manifester sous la forme d'une aortite primaire déclenchant ensuite une réponse fibroinflammatoire péri-aortique. Cette hypothèse est renforcée par l'observation d'une inflammation prédominant dans l'adventice, où l'on trouve également une vasculite des vasa vasorum ainsi que des follicules lymphoïdes avec des centres germinaux ${ }^{5,11,48}$. 


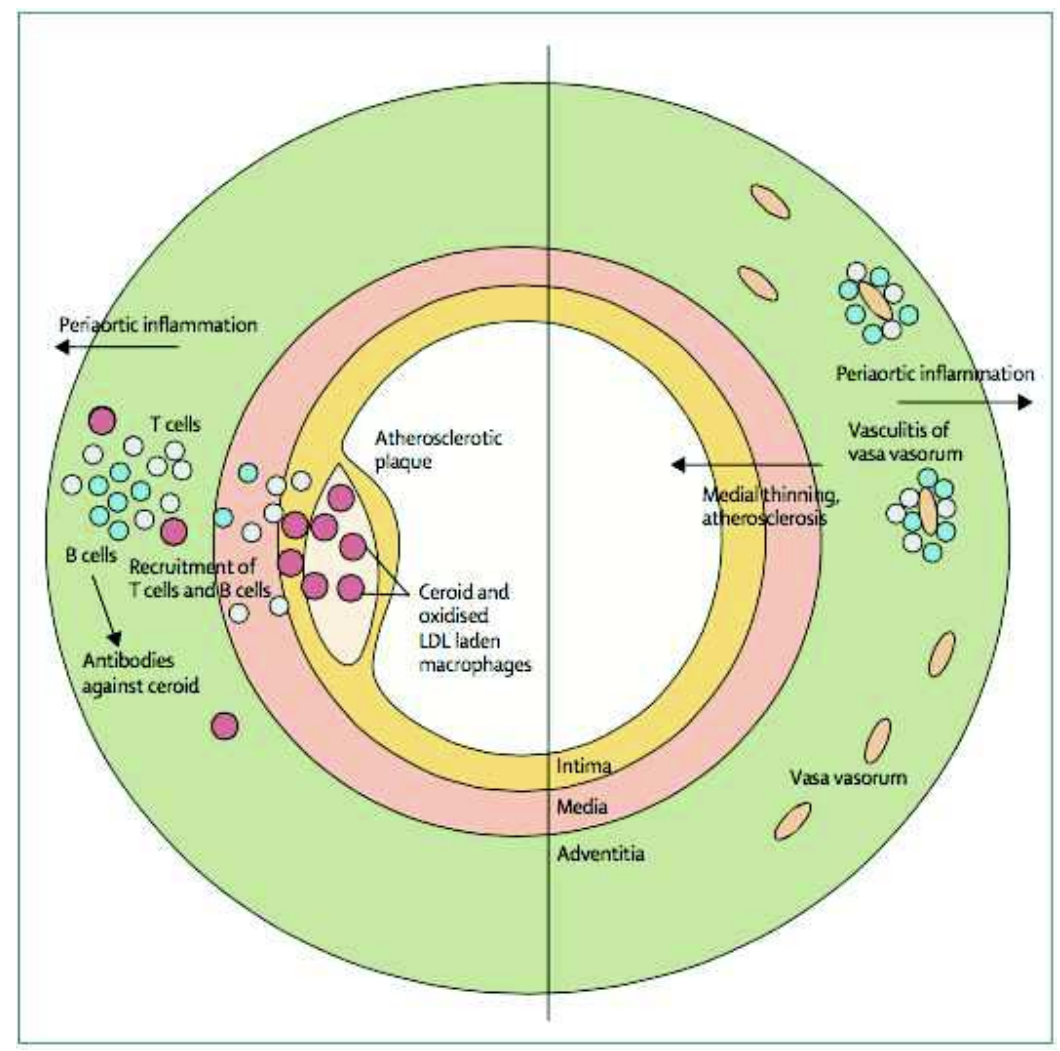

Figure 4. Représentation schématique de la physiopathologie de la FRP, hypothèse de l'aortite autoallergique en réaction aux antigènes $d^{\prime}$ 'une plaque d'athérosclérose (gauche) et hypothèse d'une péri-aortite initiée dans l'adventice (droite). (D'après Vaglio A et al. Lancet. 2006)

\section{Mécanisme immunopathologique}

Les cellules présentatrices d'antigènes présentent des antigènes aux cellules CD4+ de la paroi aortique ou du rétropéritoine environnant. Les lymphocytes T CD4+ se développent et peuvent sécréter de I'IL-6, qui est capable d'induire l'activation des cellules B et des fibroblastes ${ }^{48}$. L'importance pathogénique de l'axe médié par I'IL-6 et des lymphocytes B a été confirmée in vivo par l'efficacité des anticorps monoclonaux ciblant le récepteur de l'IL-6 (tocilizumab) et le marqueur des cellules B CD20 (rituximab), bien que ces données soient limitées à de petites séries de cas ${ }^{45,49}$.

Les cellules T CD4+ sécrètent également des cytokines Th2 telles que I'IL-4, I'IL-10, et I'IL-13, qui stimulent la prolifération des cellules $B$ et leur maturation en plasmocytes, conduisant à l'expansion préférentielle des plasmocytes IgG4+. Les cellules B sont enrichies dans les biopsies de FRPi et sont essentielles à la présentation des antigènes aux cellules Th2 ou aux cellules mémoires effectrices CD4+. Elles ont aussi un rôle important dans la persistance de la maladie ${ }^{50}$. 
Les cellules lymphoïdes sécrètent également l'éotaxine 1, qui, avec I'IL-5, entraîne le recrutement d'éosinophiles et de mastocytes, dont les produits sont capables d'induire la prolifération des fibroblastes, leur maturation en myofibroblastes et la production de collagène ${ }^{51}$.

L'activation des fibroblastes est également induite par le CCL18, un marqueur de fibrose qui est augmenté dans le sérum des patients atteints de FRPi ${ }^{52}$. Enfin, les taux sériques d'éotaxine/chemokine ligand 11 (CCL11) sont plus élevés chez les patients atteints de FRPi active que chez les témoins sains. Cette chimiokine est par ailleurs exprimée par les cellules mononuclées dans les infiltrats inflammatoires. En outre, on a constaté que le récepteur CCR3 de l'éotaxine/CCL11 était exprimé de manière diffuse par les éosinophiles, les mastocytes et les fibroblastes, ce qui suggère que cette chimiokine pourrait avoir un rôle pathogène particulier en induisant un afflux tissulaire d'éosinophiles et de mastocytes, et en stimulant directement les cellules productrices de collagène ${ }^{53}$ (figure 5).

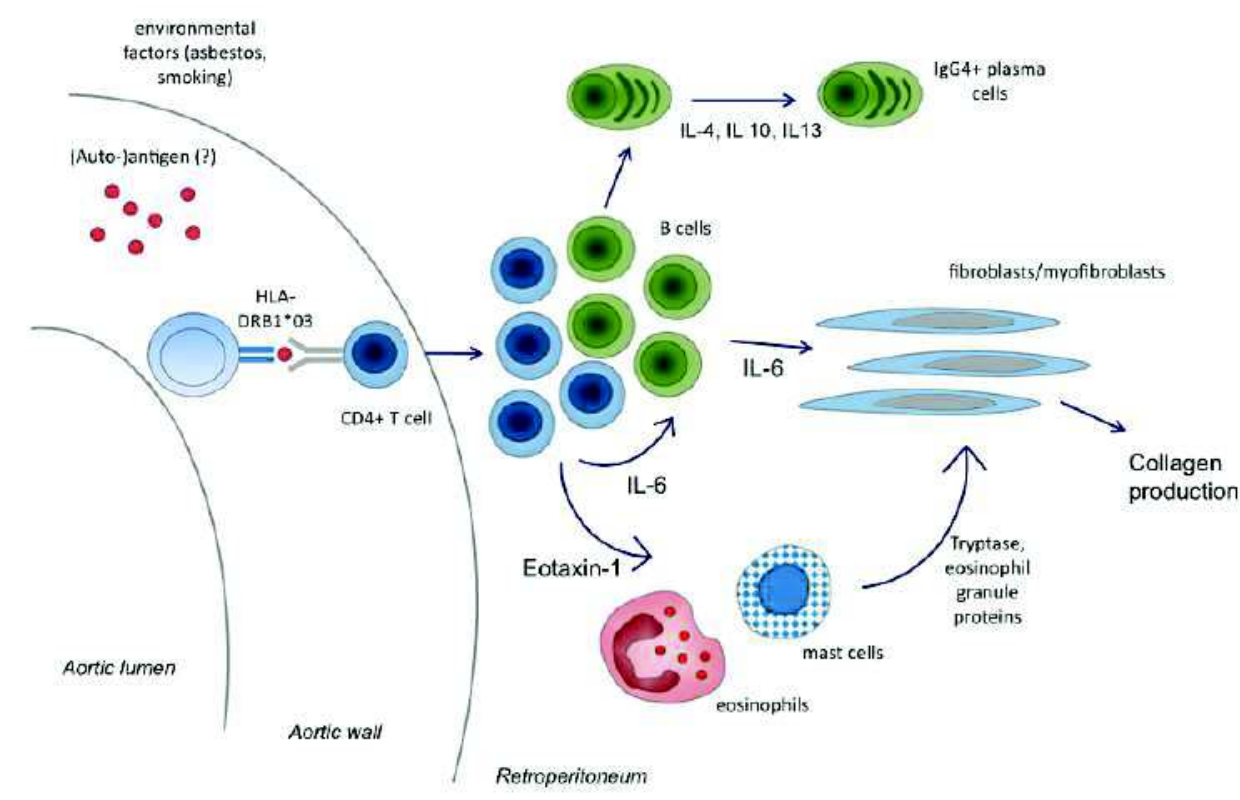

Figure 5 : Mécanismes immuno-pathologiques de la fibrose rétropéritonéale idiopathique (D'après Vaglio A et Maritati F, Journal of the American Society of Nephrology. 2016)

\section{F. Manifestations cliniques}

Les principaux symptômes de la maladie sont aspécifiques : asthénie, anorexie, amaigrissement, qui sont tous l'expression d'un état inflammatoire. L'uropathie obstructive est la complication clinique la plus fréquente de la FRP.

D'autres symptômes fréquemment rapportés sont des douleurs principalement dorsales, des flancs ou abdominales, avec ou sans constipation, ainsi que des douleurs testiculaires. Lorsque les veines 
spermatiques sont enveloppées par le processus fibrosant, une hydrocèle et une varicocèle peuvent s'observer. Des manifestations moins fréquentes peuvent également être observées à savoir l'éjaculation rétrograde, les troubles de l'érection, l'hématurie et la dysurie ${ }^{4,54}$.

\section{Complications urologiques}

L'atteinte urétérale est la complication la fréquente, elle survient dans 60 à $70 \%$ des cas ${ }^{26,55,56}$. Lorsque cette atteinte est bilatérale, elle peut provoquer une insuffisance rénale aiguë (IRA), avec le développement d'une oligo-anurie et de symptômes urémiques. Lorsqu'elle est unilatérale, l'obstruction urétérale peut évoluer de manière controlatérale des semaines à des années après le début de la maladie.

Près d'un tiers des patients présentent une hypoplasie ou une atrophie rénale en l'absence d'hydronéphrose manifeste. Dans ce contexte, on observe parfois une IRA lorsque l'uretère controlatérale est secondairement atteint ${ }^{5}$.

\section{Complications vasculaires}

La FRP se développe généralement autour de l'aorte et des artères iliaques mais la sténose de ces vaisseaux est rare, allant de 1,8 à $3 \%$ selon les séries ${ }^{56,57}$. La compression des artères iliaques peut entraîner une claudication des membres inférieurs ${ }^{58}$.

En revanche, la compression veineuse est fréquente et peut provoquer un œdème des membres inférieurs, d'autant plus si une compression lymphatique est associée. La compression des veines est généralement progressive, avec recrutement d'une collatéralité ${ }^{59}$. Les thromboses veineuses sont rares, avec un taux variant de 6 à $12 \%$ selon les séries ${ }^{26}$

La FRP peut s'étendre au pédicule vasculaire rénal, et alors être responsable d'une hypertension réno-vasculaire ${ }^{60}$.

\section{Complications génitales et abdominales}

L'implication des vaisseaux gonadiques est fréquente. L'atteinte se traduit par un gonflement du scrotum, une varicocèle et/ou une hydrocèle peuvent se développer se manifestant par une douleur testiculaire sourde ${ }^{26}$.

Par ailleurs, la constipation est un symptôme fréquent. Cependant, des manifestations abdominales plus sévères sont possibles lorsque le tissu périaortique s'étend aux artères mésentériques et cœliaques, provoquant une sténose et pouvant aboutir à des complications ischémiques ${ }^{61}$. 


\section{G. Examens biologiques}

L'anomalie biologique la plus fréquemment observée est l'augmentation des marqueurs de I'inflammation notamment la vitesse de sédimentation des érythrocytes (VS) et le taux de protéine C réactive (CRP). Ces derniers sont donc augmentés chez la majorité des patients au diagnostic. La cinétique de ces marqueurs biologiques suit l'évolution de la maladie à savoir une diminution pendant la phase de rémission et une nouvelle augmentation lors des rechutes. Une inflammation initiale plus élevée à la phase aiguë est associée à une maladie plus symptomatique, mais elle ne permet pas de prédire la réponse au traitement ni la réduction de la masse fibreuse ${ }^{62,63}$.

Une anémie modérée peut être observée, résultant de l'inflammation chronique. Le syndrome inflammatoire peut également s'accompagner d'une thrombocytose ou d'une hyperleucocytose.

La créatininémie permet de dépister une insuffisance rénale dont le mécanisme est le plus souvent obstructif, mais peut aussi être vasculaire en cas de compression de l'artère rénale. Une protéinurie ou une hématurie microscopique sont plus rarement observées.

Les facteurs anti-nucléaires (AAN) sont détectés chez $60 \%$ des patients atteints de FRPi ${ }^{9}$. Plus rarement, le facteur rhumatoïde, les anticorps anti-muscle lisse ou les ANCA sont positifs. Dans la plupart des cas, les titres des autoanticorps sont faibles et leur positivité est non spécifique. Cependant, chez certains patients, ils peuvent suggérer la présence d'une maladie auto-immune associée ${ }^{9,19,22}$. La présence d'anticorps anti-thyroïdiens signent généralement la présence d'une thyroïdite auto-immune sous-jacente, maladie auto-immune la plus fréquemment associée à la fibrose rétropéritonéale ${ }^{9}$.

II n'existe pas d'examen biologique permettant de différencier les formes secondaires des formes idiopathiques. Cependant, dans les cas de FRP secondaires d'origine néoplasique par exemple, certaines anomalies spécifiques peuvent orienter le clinicien notamment des concentrations élevées de marqueurs néoplasiques ou une hypercalcémie.

\section{H. Histologie}

\section{Fibrose rétropéritonéale idiopathique}

L'aspect histologique de la FRPi est généralement décrit comme un tissu scléreux infiltré par un mélange de cellules mononuclées, mais la proportion de ces deux composants varie selon le stade de la maladie. Au stade précoce, le tissu est souvent œdémateux et très vascularisé. II existe également une inflammation chronique active avec un infiltrat polymorphe de cellules mononuclées au sein des fibroblastes et des faisceaux de collagène ${ }^{64,65}$. Aux stades tardifs, I'histologie montre une sclérose prononcée et des calcifications éparses. 
L'infiltrat inflammatoire se compose de cellules lymphoïdes (lymphocytes B CD20 et T CD4) ${ }^{9}$, de macrophages, de plasmocytes et d'éosinophiles, les neutrophiles sont quant à eux absents. L'infiltrat peut être à la fois diffus et disposé de façon nodulaire et périvasculaire. Dans certains cas, on observe une infiltration transmurale et une nécrose fibrinoïde des vaisseaux rétropéritonéaux de petite et moyenne taille 9,64,66. (figure 6)

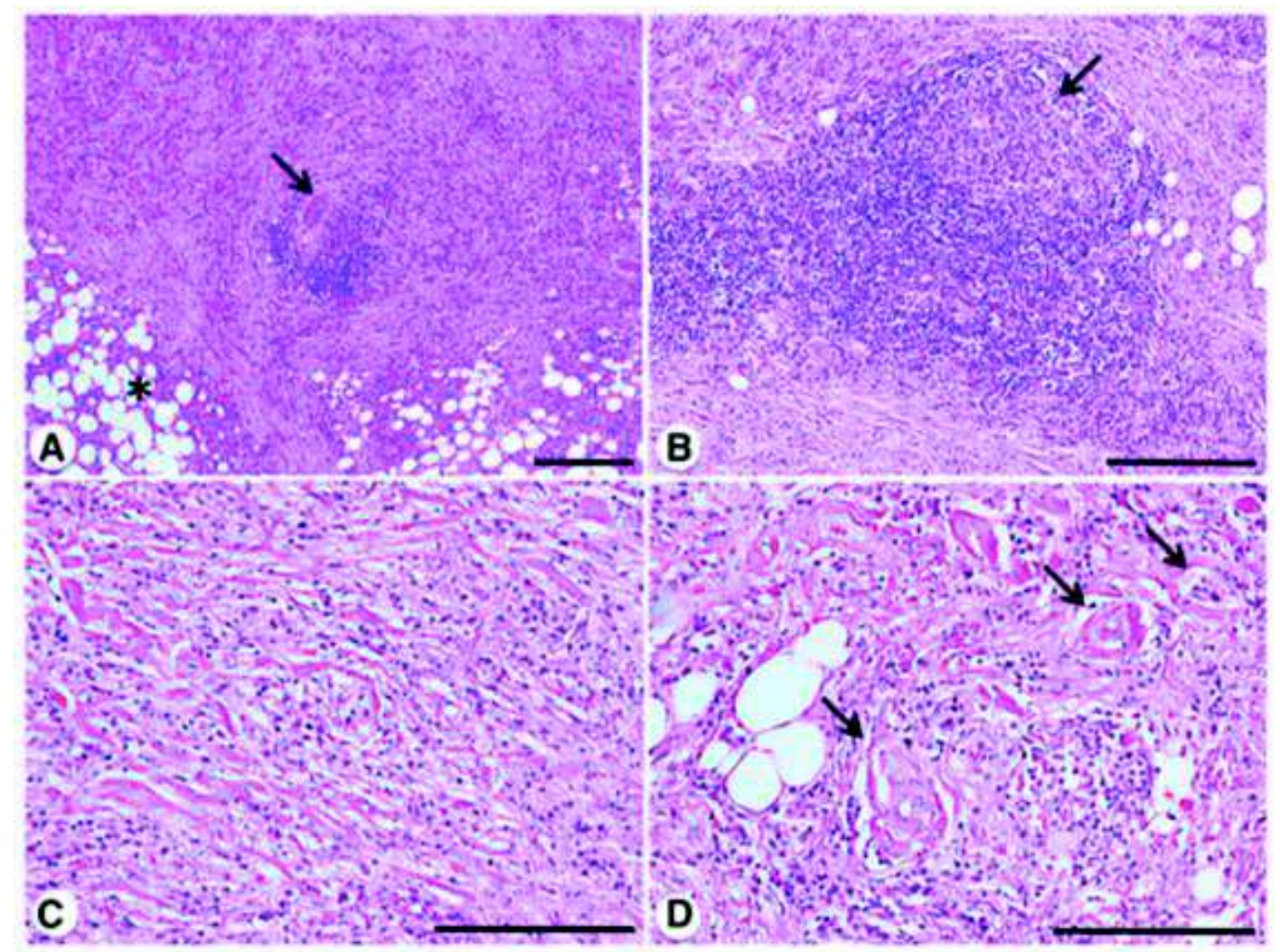

Figure 6 : Histologie de la fibrose rétropéritonéale idiopathique. A : Fibrose abondante et irrégulière remplaçant les tissus rétropéritonéaux. Infiltrat inflammatoire organisé en un agrégat lymphoïde centrée sur une artère rétropéritonéale (flèche). B : Agrégat nodulaire lymphoïde. C : Infiltrat inflammatoire diffus, composé principalement de lymphocytes et de plasmocytes dispersés dans des faisceaux de collagène. $D$ : Faisceaux de collagène entourant des vaisseaux rétropéritonéaux. ( $D$ 'après Vaglio $A$ et Maritati $F$, Journal of the American Society of Nephrology. 2016)

On observe également des modifications de la paroi aortique, notamment une dégénérescence athéroscléreuse de l'intima, un amincissement de la média et une inflammation adventitielle prononcée. L'infiltrat inflammatoire est similaire à l'infiltrat rétropéritonéal, son aspect est diffus ou disposé en agrégats nodulaires. Ces derniers sont souvent centrés sur les vasa vasorum adventitiels, qui peuvent présenter une vascularite nécrosante ${ }^{9,67}$. 


\section{Fibrose rétropéritonéale secondaire}

L'aspect histologique de la plupart des formes secondaires est identique à celui de la maladie idiopathique. Cependant, un examen microscopique attentif révèle généralement des cellules néoplasiques dispersées dans l'abondant tissu fibreux. L'invasion et la perturbation des structures musculaires et osseuses voisines ne sont pas rares dans ce type de maladies secondaires. Dans le cas des lymphomes, l'infiltrat inflammatoire peut être monoclonal ${ }^{68}$.

La présence de granulomes peut suggérer une infection sous-jacente, par exemple la tuberculose.

Les dépôts d'hémosidérine indiquent généralement une hémorragie, qui peut à son tour être le résultat d'un traumatisme.

La présence de lipoblastes peut suggérer le diagnostic de liposarcome bien différencié avec des caractéristiques sclérosantes et inflammatoires.

La fibrose rétropéritonéale post-radique a un aspect plutôt cicatriciel, le tissu est principalement scléreux, avec un contingent cellulaire faible ${ }^{69,70}$.

Enfin, la maladie d'Erdheim-Chester est caractérisée par des quantités variables d'histiocytes "spumeux" (systématiquement positifs pour CD68KP1 et négatifs pour CD1a) dans un tissu collagène dense 71

I. Imagerie

\section{1. Échographie}

En échographie, la FRP est visualisée comme une masse rétropéritonéale hypo ou isoéchogène, délimitée mais aux contours irréguliers, en avant du rachis lombaire ou du promontoire du sacrum. L'extension caudale au-delà du promontoire du sacrum et l'absence de lobulation ont été identifiées comme des indices d'une cause bénigne, bien qu'il s'agisse de signes non spécifiques, qui ne peuvent pas être utilisés pour exclure la malignité ${ }^{72}$. L'échographie, est rapide et rentable pour détecter une hydronéphrose, elle est donc utile en première ligne. Elle permet également le dépistage d'une dilatation ou d'un anévrysme aortique.

\section{Tomodensitométrie}

La radiographie conventionnelle n'est d'aucune aide pour diagnostiquer la maladie, alors que la tomodensitométrie (TDM) et l'imagerie par résonance magnétique (IRM) sont les outils d'imagerie les plus appropriés. 
La présentation typique scannographique de la FRP est une masse de tissu péri-aortique bien délimitée mais irrégulière, s'étendant des artères rénales aux vaisseaux iliaques et plus loin, généralement située antérieurement et latéralement à l'aorte (figure 7). La masse peut également s'étendre aux organes adjacents (duodénum, reins) mais dans de tels cas, l'exclusion d'une étiologie maligne devient obligatoire ${ }^{58}$.

La densité du tissu fibreux est similaire à celle du muscle psoas. Après injection de produit de contraste, le rehaussement varie en fonction du stade de la maladie. Il est important dans les stades précoces et faible ou absent dans les stades tardifs inactifs ${ }^{73}$.

La tomodensitométrie est également utile pour détecter d'autres complications observées au cours de la FRP : hydronéphrose, thrombose veineuse profonde ou atteinte des vaisseaux rénaux.

Une adénopathie localisée à proximité de la masse fibreuse est observée dans près d'un quart des cas. II s'agit généralement de ganglions lymphatiques infracentimétriques, vraisemblablement réactionnels au processus inflammatoire, qui ne doivent pas faire suspecter de malignité ${ }^{4}$. A l'inverse, le refoulement de l'aorte et de la veine cave inférieure dû à des adénomégalies postérieures à ces vaisseaux est suspect de malignité ${ }^{74}$.

Figure 7 : Images en tomodensitométries de fibrose rétropéritonéales idiopathiques. (D’après Vaglio $A$ et Maritati F, Journal of the American Society of Nephrology. 2016)
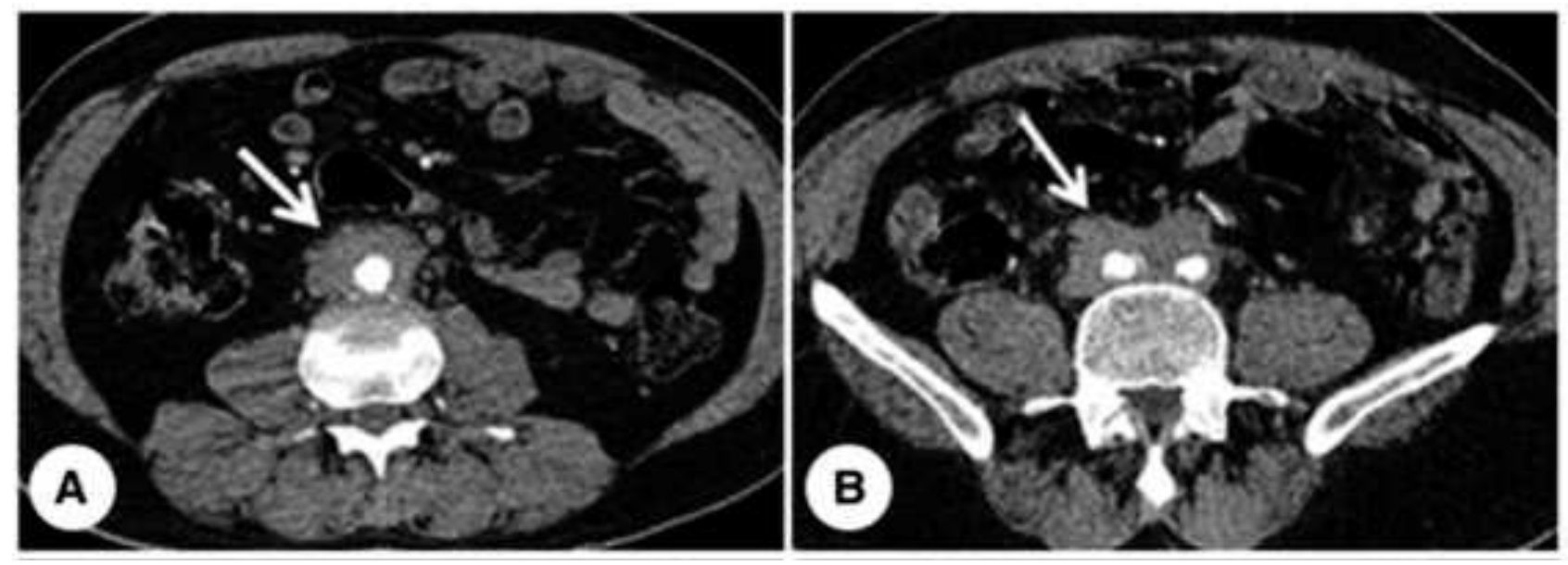

A et $B$ : FRPi typique se développant antérieurement et latéralement par rapport à l'aorte abdominale (A) et des deux artères iliaques (B). 


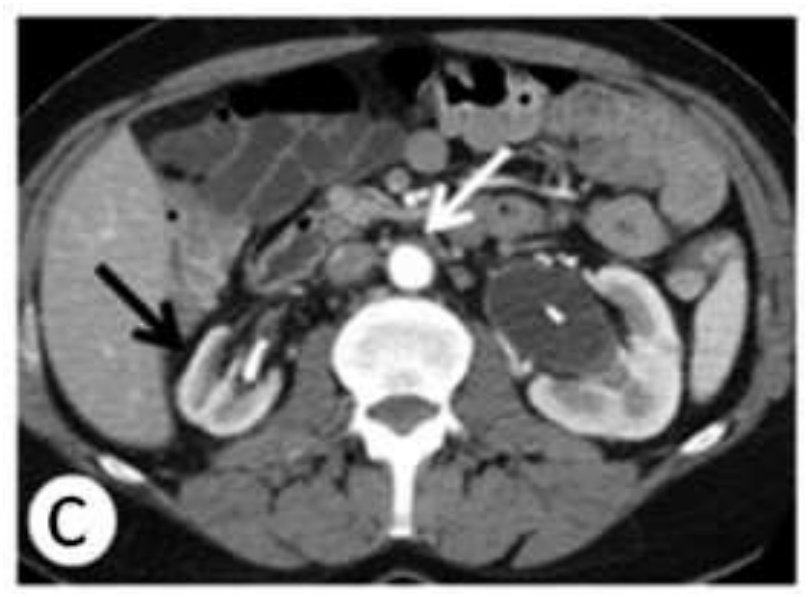

C : Hypoplasie marquée du rein droit et hydronéphrose sévère du rein gauche dans un contexte de FRPi.
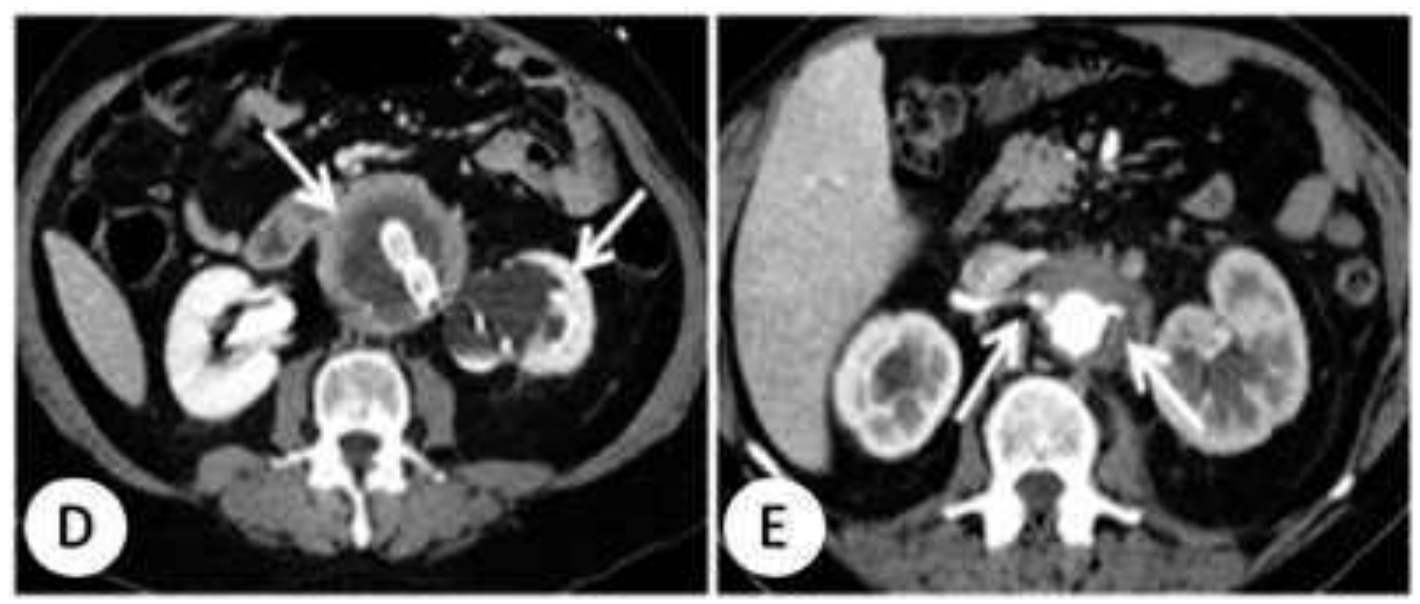

$D$ : FRPi péri-anévrysmale et hydronéphrose sévère du rein gauche

E : FRPi avec engainement des origines des deux artères rénales (flèches)

\section{Imagerie par résonnance magnétique}

En pratique clinique, l'imagerie par résonnance magnétique (IRM) est équivalente à la TDM pour le diagnostic et le suivi de la FRP. L'IRM évite le recours à un produit de contraste néphrotoxique, mais elle est moins sensible aux calcifications vasculaires. L'IRM peut fournir une meilleure définition de la FRP par rapport aux tissus environnants, principalement après suppression du signal de graisse.

Les caractéristiques IRM typiques sont la faible intensité du signal de la masse sur les images pondérées en T1 et une intensité variable sur les images pondérées en T2, reflétant le degré d'inflammation active (figure 8). Plus la lésion est active, plus le signal est intense sur les images pondérées en T2 et, en cas 
d'administration de gadolinium, le renforcement du contraste est d'autant plus élevé. Ceci s'explique par la présence d'un œdème tissulaire et d'une hypercellularité. Lorsque la maladie est en rémission, le rehaussement par le gadolinium est généralement faible ou absent ${ }^{58}$.
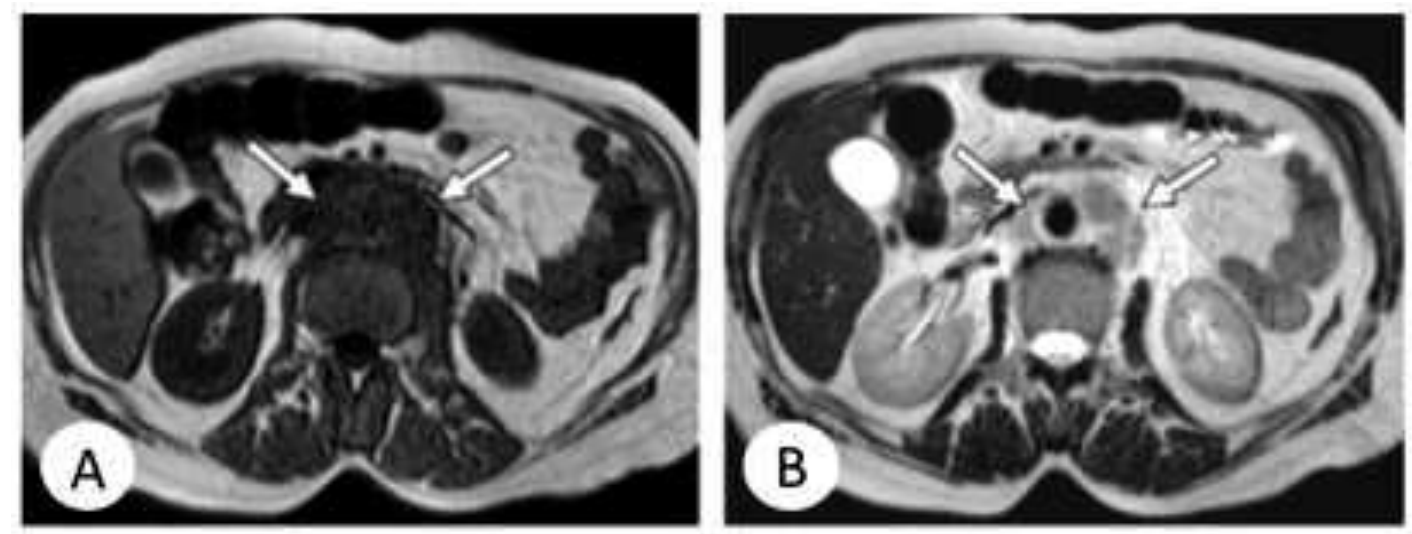

Figure 8 : Fibrose rétropéritonéale idiopathie en IRM pondérée en T1 (A) et T2 (B) ( $D$ 'après Bakir et al. Radiology. 2014)

\section{PET TDM}

Le 18FDG-PET/CT permet une quantification assez précise de l'activité métabolique de la FRP (figure 9), aidant ainsi à la fois au diagnostic et au suivi. Il apparaît même plus performant que le dosage de la CRP 75

Certains auteurs ont montré que les patients n'ayant pas d'hyperfixation au PET TDM ne bénéficiaient pas d'une corticothérapie et qu'une réponse pouvait être attendue uniquement chez des patients ayant une maladie métaboliquement active ${ }^{76}$.

Cependant, dans une étude publiée sur le rôle pronostique du PET scanner, il a été montré que les patients sans hyperfixation ont seulement des taux de réponse plus faibles, mais pas nuls. De plus, le degré de fixation n'était pas associé à des taux de réponse différents ${ }^{77}$.

Compte tenu de sa sensibilité élevée la TEP-TDM au 18FDG peut également montrer une fixation intense des lésions néoplasiques et infectieuses, ce qui limite donc sa spécificité diagnostique.

Néanmoins, cette technique d'imagerie peut également être utile sur le plan clinique car elle peut détecter des lésions métaboliquement actives occultes et révéler d'autres sites impliqués dans des formes de maladies systémiques (par exemple, périaortite thoracique, médiastinite fibrosante) ${ }^{25,78}$. 

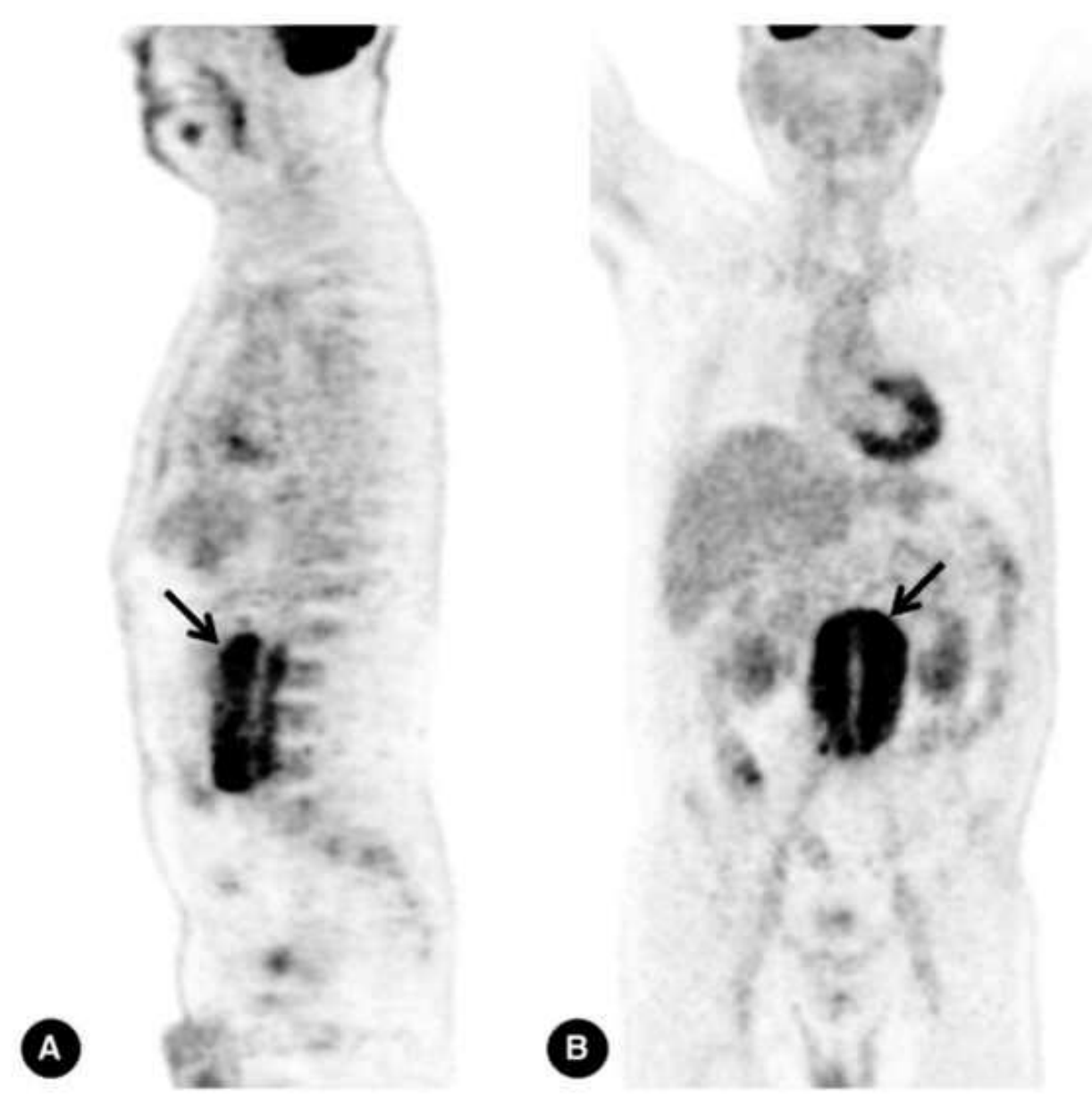

Figure 9 : PET TDM au 18 FDG chez un patient atteint de FRPi. Fixation intense autour de l'aorte abdominale. (D’après Vaglio A et Maritati F, Journal of the American Society of Nephrology. 2016)

\section{Diagnostics différentiels en imagerie}

Les aspects morphologiques de la FRP au scanner ou à l'IRM peuvent aider le médecin à différencier les formes idiopathiques des formes secondaires. Les formes idiopathiques sont généralement caractérisées par des densités homogènes avec une infiltration périphérique alors que la prolifération des formes secondaires est généralement nodulaire et lobulée ${ }^{72}$.

La FRPi, à la différence des lymphomes et des métastases, est généralement située en deçà du hile rénal, antérieurement et latéralement par rapport à l'aorte qui n'est pas déplacée vers l'avant, et il existe une attraction médiale des uretères.

Ainsi, un déplacement antérieur de l'aorte abdominale et des vaisseaux iliaques et un refoulement latéral des uretères causés par la masse, ainsi qu'un signal inhomogène sur les images pondérées en T2, doivent renforcer la suspicion d'une forme maligne ${ }^{79}$. 
Les cancers se présentent généralement sous la forme d'une masse fibreuse disloquant les muscles psoas ou détruisant l'os alors que la FRPi ne présente aucune de ces caractéristiques ${ }^{80}$.

Des adénopathies localisées, adjacentes à la FRP s'observent dans les formes idiopathiques, mais lorsqu'elles deviennent confluentes et tendent à entourer les gros vaisseaux, elles sont suspectes de malignité.

La maladie d'Erdheim-Chester doit être suspectée lorsque la fibrose péri-aortique est associée à une fibrose péri-rénale symétrique et bilatérale, appelée « reins chevelus ${ }^{81}$.

\begin{tabular}{|c|c|c|c|}
\hline & $\begin{array}{l}\text { FRP primitive } \\
\text { Phase précoce }\end{array}$ & $\begin{array}{l}\text { FRP primitive } \\
\text { Phase tardive }\end{array}$ & FRP maligne \\
\hline \multirow{3}{*}{$\begin{array}{l}\text { Morphologie - } \\
\text { Localisation }\end{array}$} & $\begin{array}{l}\text { Masse périaortique +/- } \\
\text { aspect symétrique }\end{array}$ & $\begin{array}{l}\text { Masse périaortique }+/ \text { - } \\
\text { aspect symétrique }\end{array}$ & $\begin{array}{l}\text { Masse périaortique } \\
\text { aspect asymétrique }\end{array}$ \\
\hline & $\begin{array}{c}\text { Absence de déviation de } \\
\text { l'aorte }\end{array}$ & $\begin{array}{l}\text { Absence de déviation de } \\
\text { l'aorte }\end{array}$ & $\begin{array}{l}\text { Déviation antérieur de } \\
\text { l'aorte }\end{array}$ \\
\hline & $\begin{array}{c}\text { Masse bien délimitée en } \\
\text { antérieur } \\
\text { Aspect linéaire ou } \\
\text { courbé }\end{array}$ & $\begin{array}{l}\text { Masse bien délimitée en } \\
\text { antérieur } \\
\text { Aspect linéaire ou } \\
\text { courbé }\end{array}$ & $\begin{array}{c}\text { Masse bien délimitée en } \\
\text { antérieur } \\
\text { Aspect nodulaire }\end{array}$ \\
\hline \multirow[t]{2}{*}{ Extension } & $\begin{array}{c}\text { Déviation médiale des } \\
\text { uretères }\end{array}$ & $\begin{array}{c}\text { Déviation médiale des } \\
\text { uretères }\end{array}$ & $\begin{array}{c}\text { Déviation latérale des } \\
\text { uretères }\end{array}$ \\
\hline & $\begin{array}{c}\text { Extension crâniale et/ou } \\
\text { inférieure }\end{array}$ & $\begin{array}{l}\text { Extension crâniale et/ou } \\
\text { inférieure }\end{array}$ & $\begin{array}{c}\text { Extension latérale vers le } \\
\text { muscle psoas et organes } \\
\text { de voisinage }\end{array}$ \\
\hline Séquence T2 & Variable & Hypo-intense & Hyper-intense \\
\hline Rehaussement & Intense & Faible & Variable, hétérogène \\
\hline Cartographie ADC & Réduction & $\begin{array}{c}\text { Réduction faible ou } \\
\text { absente }\end{array}$ & Réduction intense \\
\hline
\end{tabular}

Tableau 3 : Résumé des caractéristiques radiologiques, distinction FRP idiopathique et FRP maligne

\section{J. Diagnostic de fibrose rétropéritonéale idiopathique}

II n'existe pas de critères diagnostiques établis pour la fibrose rétropéritonéale idiopathique et le diagnostic est un diagnostic d'exclusion.

Il convient par conséquent d'éliminer les causes de FRP secondaires mentionnées précédemment, ce qui n'est pas toujours évident dans le cas des tumeurs malignes ou de certaines infections comme la tuberculose. Il est proposé d'exclure à titre systématique la tuberculose, I'hépatite B, I'hépatite C, I'infection par le VIH et la syphilis. En cas d'atteinte péri-rénale, très suspecte pour la maladie d'Erdheim-Chester, une scintigraphie osseuse devra également être réalisée ${ }^{82}$. 
La TDM et l'IRM sont toutes deux de bons moyens diagnostiques, mais lorsque la FRP se présente de manière atypique, une biopsie de la masse est recommandée ${ }^{72,83}$. La biopsie du tissu n'est pas toujours réalisable en raison de la localisation de la fibrose. La technique de biopsie varie entre les approches ouvertes, laparoscopiques et guidées par scanner. Le pourcentage de cas prouvés par biopsie varie largement selon les séries, de $24 \%$ à $77 \%{ }^{54,84,85}$.

\section{K. Traitement}

\section{Prise en charge urologique}

Le premier objectif du traitement est la levée de l'obstruction urétérale. Cet objectif était autrefois obtenu grâce à des procédures chirurgicales invasives (par exemple l'urétérolyse), qui sont désormais réservées à certains cas particuliers. Actuellement, les procédures conservatrices (endoprothèses double J, néphrostomies) sont préférées ${ }^{60}$.

La pose d'une endoprothèse urétérale permet une meilleure qualité de vie que la néphrostomie et est généralement un succès. Cependant, les endoprothèses et les néphrostomies ont des taux de complications comparables (infection, obstruction) ${ }^{86}$.

En revanche, si l'approche conservatrice est inefficace, il devient nécessaire de pratiquer une urétérolyse chirurgicale, suivie d'une transposition intrapéritonéale et d'un enveloppement omental des uretères. Cette opération est généralement réalisée par une approche ouverte traditionnelle. Cependant, certains auteurs ont rapporté des résultats favorables en utilisant la laparoscopie, qui, dans une série rétrospective, a été associée à une durée d'hospitalisation plus courte et à des besoins transfusionnels réduits par rapport à l'approche ouverte ${ }^{87}$. Par rapport à la stratégie conservatrice, l'urétérolyse chirurgicale présente l'avantage évident de permettre la réalisation de biopsies multiples.

Une approche pas à pas du traitement est ainsi proposée, prenant en compte la gravité de I'obstruction urétérale comme première évaluation ${ }^{26}$. Bien qu'il n'existe pas de recommandations établies, lorsque l'obstruction urétérale est légère et qu'il n'y a pas d'altération de la fonction rénale, il semble recommandé de commencer un traitement médical sans drainage urinaire. En cas d'obstruction urétérale modérée à sévère et/ou d'insuffisance rénale, le drainage urétéral doit être la priorité, suivi du traitement médical. 


\section{Prise en charge médicale des FRPi}

Les traitements immunosuppresseurs de première ligne sont représentés par les corticostéroïdes afin d'obtenir une rémission. La rémission est définie par la résolution de l'hydronéphrose, le soulagement des symptômes, la normalisation des marqueurs biologiques inflammatoires et la réduction de la masse à l'imagerie. Les glucocorticoïdes permettent généralement d'obtenir une rémission rapide. La masse inflammatoire diminue davantage au cours des premiers mois de traitement, tandis qu'elle se stabilise généralement dans les phases tardives ${ }^{5}$.

Aucun facteur pronostique de bonne réponse n'a été identifié jusqu'à présent, cependant un traitement précoce paraît important une fois le diagnostic confirmé. En effet, les stades tardifs de la maladie sont caractérisés par une prédominance du tissu fibreux sur le tissu inflammatoire.

\section{a. Corticothérapie}

Aucun essai contrôlé randomisé n'a été mené, bien que plusieurs études aient explorées l'efficacité des corticoïdes. Kardar et al ont traité 11 patients consécutifs atteints de FRPi avec de la prednisone à une dose initiale de $60 \mathrm{mg}$ un jour sur deux pendant 2 mois, diminuée sur 2 mois à $5 \mathrm{mg}$ par jour. Neuf de ces patients ont présenté une rémission de la maladie, le traitement étant poursuivi jusqu'à 2 ans ${ }^{88}$. Van Bommel et al ont publié des données sur 24 patients traités avec des stéroïdes à une dose initiale de $60 \mathrm{mg}$ par jour pendant 6 semaines et diminuée sur les 2-3 mois suivants à $10 \mathrm{mg}$ par jour. La durée totale du traitement était de 1 an. Vingt deux des 24 patients ont obtenu une rémission, bien que des rechutes aient été observées chez 13 d'entre eux ${ }^{89}$.

La corticothérapie doit donc être initiée dès que possible. Le traitement d'induction avec l'utilisation de fortes doses de prednisone ( $1 \mathrm{mg} / \mathrm{kg} / \mathrm{jour}$ pendant le premier mois) est la meilleure option pour réduire I'activité de la maladie. Après un mois de traitement, une réévaluation de l'activité de la maladie (symptômes cliniques, CRP) ainsi qu'une évaluation morphologique de la masse sont recommandées. Si la rémission est obtenue, la prednisone peut être progressivement réduite à 5-10 mg par jour en 3-4 mois, puis maintenue pendant 6-9 mois supplémentaires. Une tentative de retrait des endoprothèses urétérales doit être effectuée une fois que la rémission clinico-biologique a été obtenue et que l'imagerie a démontré une réduction de la masse $^{84}$. 


\section{b. Tamoxifène}

Le tamoxifène est un agent anti-oestrogène avec une activité anti-fibrotique potentielle. II a été proposé comme alternative aux glucocorticoïdes ${ }^{90}$, en particulier chez les patients présentant une toxicité ou une contre-indication à la corticothérapie ${ }^{85,91}$.

L'équipe de Van Bommel et al a publié une étude portant sur 19 patients atteints de FRPi montrant que la plupart d'entre eux a bénéficié de cette approche. L'utilisation de tamoxifène s'est accompagnée d'une réduction de masse chez $71 \%$ des patients au $4{ }^{\text {ème }}$ mois et $85 \%$ au $8^{\text {ème }}$ mois, avec une bonne tolérance

${ }^{90}$. Cependant, dans un essai contrôlé randomisé, un traitement de 8 mois par tamoxifène s'est avéré significativement moins efficace qu'un traitement par prednisone de même durée pour maintenir la rémission chez des patients traités en induction par prednisone $(1 \mathrm{mg} / \mathrm{kg} \text { par jour pendant } 1 \text { mois })^{84}$.

Par conséquent, à ce jour, l'efficacité du tamoxifène n'est pas confirmée par des essais contrôlés et sa supériorité par rapport aux corticostéroïdes n'est pas prouvée. Cependant cette molécule peut être une alternative pour les patients présentant des contre-indications à l'utilisation des glucocorticoïdes.

\section{c. Traitements de seconde ligne}

Lorsque la rémission n'est pas obtenue, ou que la toxicité liée aux glucocorticoïdes est inacceptable, l'ajout d'un second immunosuppresseur est conseillé. II n'existe pas de preuves claires soutenant l'idée que ces agents agissent en synergie avec les stéroïdes. Ils pourraient en effet simplement fonctionner comme des traitements d'épargne cortisonique.

Les expériences rapportées dans la littérature concernent principalement le mycophénolate mofétil ${ }^{92-95}$, le méthotrexate ${ }^{96,97}$, l'azathioprine ${ }^{98}$ ou le cyclophosphamide ${ }^{99}$.

Le méthotrexate a été utilisé avec succès comme traitement d'entretien à visée d'épargne cortisonique. Un essai a inclus 16 patients atteints d'une maladie récidivante et traités en add-on par méthotrexate (15-20 mg/semaine) pendant 12 mois associé à de la prednisone à la dose initiale de 0,5-1 $\mathrm{mg} / \mathrm{kg} /$ jour pendant ces 12 mois. La majorité des patients (79\%) était en rémission au $12^{\text {ème }}$ mois, avec survie sans rechute plus longue si le traitement était poursuivi au-delà de 12 mois $^{96}$.

Le mycophénolate mofétil est largement utilisé, notamment en raison de sa bonne tolérance et de l'absence de contre-indications chez les patients souffrant d'insuffisance rénale. Ajouté à la prednisone, il s'est avéré efficace comme traitement initial et peut être envisagé chez certains patients résistant aux corticoïdes $^{95,100}$. Il a été administré chez 28 patients à la dose de $1 \mathrm{~g}$ deux fois par jour pour une durée moyenne de 24 mois, associé à de la prednisone à une dose initiale de 40 mg/jour diminué progressivement 
sur une période de 6 mois. Une réduction des signes clinico-biologiques a été observée chez l'ensemble des patients et une réduction de la masse en imagerie chez $89 \%$ des patients.

Le cyclophosphamide a également été utilisé efficacement comme traitement initial suivi d'un entretien avec d'autres immunosuppresseurs, mais il n'est actuellement pas recommandé comme traitement de première ligne ${ }^{99,101}$. Sur une cohorte rétrospective de 35 patients, 21 patients ont reçu un traitement d'induction par cyclophosphamide. II a été observé pour l'ensemble d'entre eux une réduction significative du diamètre transverse de la masse en imagerie ainsi qu'un raccourcissement du délai d'ablation des sondes JJ (9,6 $\pm 4,4$ mois versus 19,3 $\pm 3,2$ mois pour les autres traitements).

Dans les cas réfractaires, lorsque l'ajout d'un second immunosuppresseur ne s'avère pas efficace, de nouvelles stratégies thérapeutiques consistent en l'utilisation de biothérapies comme le rituximab ou le tocilizumab. Bien qu'il existe une base physiopathologique pour le rituximab (anticorps anti-CD20 déplétant les cellules B) et le tocilizumab (anticorps anti-récepteur de l'interleukine 6), les données concernant leur efficacité sont encore rares ${ }^{45,102}$. Le rituximab a également été utilisé avec succès chez des patients présentant une maladie liée aux $\lg G 4$, avec ou sans périaortite. ${ }^{46}$

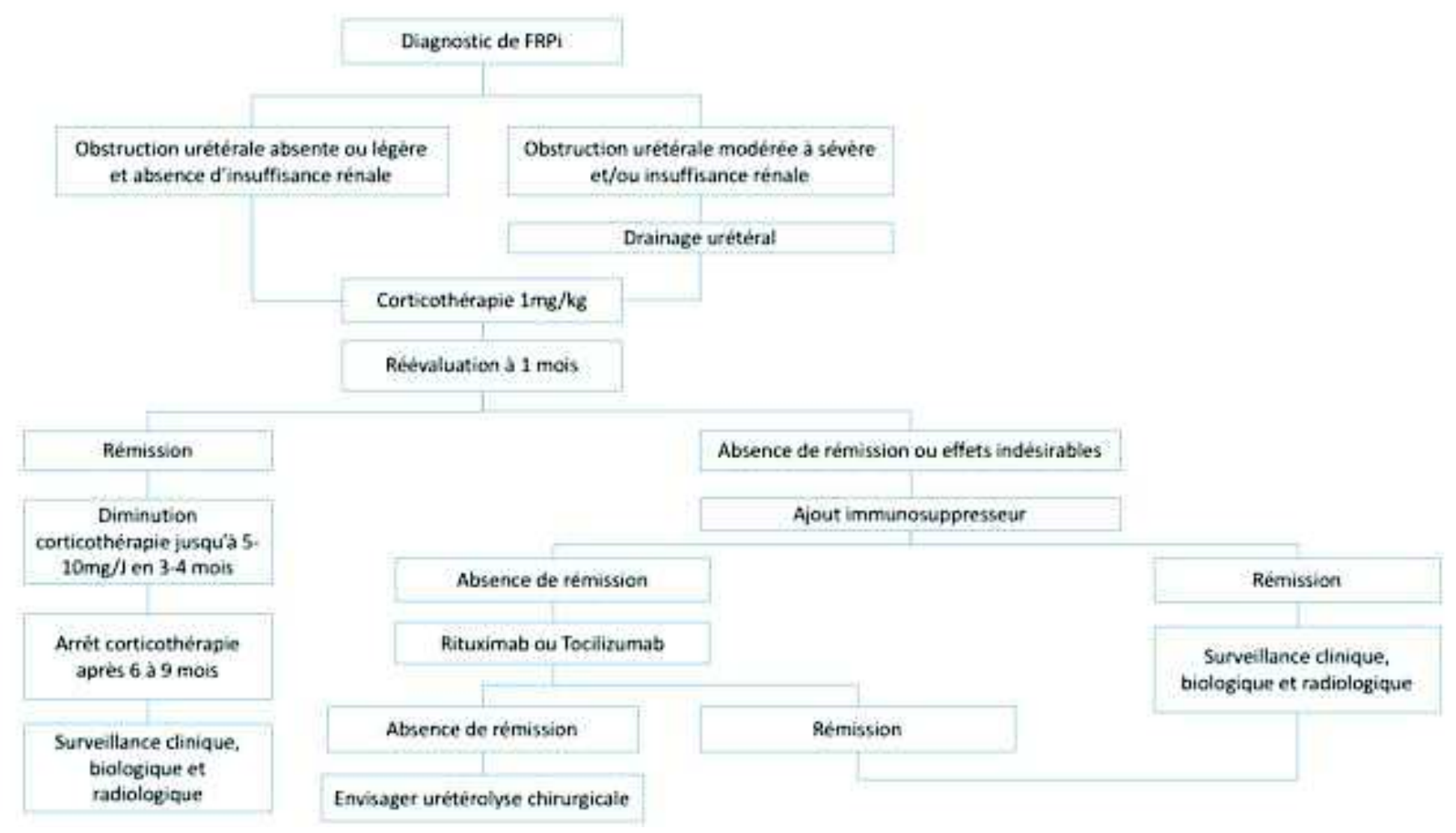

Figure 10 : Algorithme de prise en charge de la FRPi ( $D$ 'après Vaglio A et Maritati $F$, Journal of the American Society of Nephrology. 2016) 


\section{Prise en charge de la fibrose rétropéritonéale secondaire}

Le traitement des formes secondaires de FRP est en lien avec l'étiologie. La place de la corticothérapie n'est pas définie pour ces FRP (cancers, traumatismes, chirurgies majeures, radiothérapie). La chirurgie palliative est parfois envisagée. La FRP tumorale est de mauvais pronostic, avec une survie moyenne de seulement 3 à 6 mois. La fibrose rétropéritonéale secondaire à la maladie d'Erdheim-Chester nécessite également des procédures interventionnelles afin de lever l'obstruction urétérale, suivies de thérapies médicales spécifiques, qui sont maintenant basées sur l'utilisation d'interféron, de diverses thérapies immunosuppressives et, chez les patients ayant la mutation V600E du proto-oncogène BRAF, de I'inhibiteur BRAF vemurafenib.

\section{L. Évolution et suivi}

Comme c'est le cas pour la plupart des maladies inflammatoires, l'évolution de la FRPi est chronique et récidivante. Les taux de rechute varient largement d'une étude à l'autre, allant de $10 \%$ à $72 \%$, probablement en raison de différences dans les protocoles de traitement, la durée de la thérapie et le suivi 89,103

Le suivi doit donc être rapproché, avec une surveillance clinique, biologique et une échographie périodique pour exclure une hydronéphrose, une imagerie (scanner le plus souvent, ou IRM) pour définir la taille et les changements morphologiques de la masse, ainsi qu'une TEP au 18FDG pour évaluer précisément l'activité de la maladie et l'efficacité du traitement.

Aucun facteur pronostique de rechute n'a été clairement démontré. Un taux de rechute de 10 à 50\% est rapporté après l'interruption du traitement ${ }^{104}$. Les rechutes répondent généralement à la réintroduction de la corticothérapie, au prix d'effets secondaires potentiels inhérents à cette thérapeutique.

Malgré la nature récidivante de la maladie, l'issue est généralement bénigne, avec un faible taux de mortalité (3-7\%) ${ }^{104}$. Alors que l'insuffisance rénale chronique (IRC) survient chez un tiers des patients, l'insuffisance rénale terminale (IRT) nécessitant une dialyse et/ou une transplantation rénale est rare ${ }^{54,84}$. Les facteurs de risque de développement de I'IRC ont été évalués dans une étude française, rétrospective multicentrique, qui a identifié l'âge au début de la maladie, la présence d'un diabète et les valeurs initiales de la créatinine comme facteurs prédictifs de I'IRC ${ }^{105}$. 


\section{Objectif de l'étude}

La conduite à tenir devant un tableau clinique et paraclinique compatible avec le diagnostic de FRP n'est pas consensuelle, tout comme la prise en charge thérapeutique. Après avoir évoqué le diagnostic de FRP, se pose la question du diagnostic différentiel. Il faut ensuite éliminer les causes secondaires, et rechercher les maladies associées parmi lesquelles la maladie associée aux IgG4. La prise en charge thérapeutique n'est pas codifiée et repose avant tout sur la corticothérapie générale.

Les objectifs de cette étude sont d'évaluer les caractéristiques cliniques et paracliniques des patients atteints de FRP idiopathiques ou secondaires, ainsi que leur prise en charge thérapeutique au CHU de Rouen. 


\section{Patients et méthode}

\section{A. Inclusion des patients}

Il s'agit d'une étude observationnelle, rétrospective et monocentrique menée au sein du CHU de Rouen sur une période allant de 2010 à 2021, dans les services de médecine interne, urologie et néphrologie.

Les patients ont été identifiés grâce à l'entrepôt de données de santé du CHU « Doc'EDS » avec comme mot clé « fibrose rétropéritonéale ».

Les critères d'inclusion étaient les suivants :

- $\quad$ Âge $>18$ ans

- FRP idiopathique ou secondaire, fibrose péri-anévrysmale de l'aorte abdominale, anévrysme inflammatoire de l'aorte abdominale

Les critères d'exclusion étaient les suivants :

- Aortite, anévrysme de l'aorte abdominale non inflammatoire

- Suivi en dehors du CHU de Rouen

- Données insuffisantes (perdus de vue, absence de suivi, FRP mentionnée comme antécédent)

\section{B. Diagnostic de fibrose rétropéritonéale}

En l'absence de critère diagnostique consensuel dans la littérature, le diagnostic de FRP reposait essentiellement sur l'imagerie qui mettait en évidence une infiltration régulière et homogène de densité tissulaire, périvasculaire, engainant l'aorte abdominale plus ou moins étendue aux vaisseaux iliaques et pouvant prendre le contraste après injection. Ce diagnostic était renforcé par une symptomatologie évocatrice et/ou un syndrome inflammatoire biologique.

La preuve histologique n'était pas obligatoire et lorsqu'un prélèvement histologique avait été réalisé, le diagnostic de FRP était retenu en présence d'un tissu fibro-inflammatoire polymorphe composé d'un infiltrat plus ou moins dense en cellules lymphoïdes, plasmocytaires, de macrophages et de polynucléaires éosinophiles. Aucun marqueur immunohistochimique spécifique n'était précisé. Compte tenu de l'identification récente de l'entité « maladie associée aux lgG4 », le marquage lgG4 n'était pas exigé. 


\section{Recueil de données}

Après sélection des patients atteints de fibrose rétropéritonéale, les informations ont été recueillies dans le dossier informatisé : données cliniques et biologiques, comptes rendus de consultations et d'hospitalisations et données d'imageries.

Pour chaque patient, les éléments suivants ont été recueillis :

- Le sexe et l'âge au moment du diagnostic

- Les comorbidités et facteurs de risque de FRP : facteurs de risques cardio-vasculaires (IMC, tabagisme actif ou sevré depuis au moins 3 ans, diabète, dyslipidémie, hypertension artérielle), antécédent d'exposition à l'amiante, d'atteinte ischémique vasculaire, de chirurgie intra-abdominale, la présence d'un cancer connu ou d'un traitement par radio-chimiothérapie

- La présence d'un anévrysme ou d'une ectasie de l'aorte abdominale

- $\quad$ Le type de FRP : idiopathique ou secondaire

- Dans le cas des FRP secondaires, l'étiologie : néoplasique, infectieuse, post-radique, postchirurgicale, médicamenteuse

- La recherche ou non d'une maladie associée aux IgG4 ainsi que la classification selon les critères « CDC » le cas échéant : MAG4 possible, probable ou certaine

- La présence d'une pathologie associée : spondylarthrite ankylosante, lupus, vascularite des petits vaisseaux, polyarthrite rhumatoïde, thyroïdite auto-immune, psoriasis

- Les données cliniques : présence de symptômes systémiques (anorexie, asthénie, amaigrissement, fébricule/fièvre), de douleurs lombaires ou abdominales, de symptômes de colique néphrétique, d'une dysurie, de manifestations testiculaires (douleur, varicocèle ou hydrocèle), d'œdèmes des membres inférieurs ou d'une claudication intermittente des membres inférieurs

- Les données biologiques : NFS, présence d'un syndrome inflammatoire biologique (CRP > 5mg/L ou fibrinogène $>4 \mathrm{~g} / \mathrm{L}$, d'une insuffisance rénale aigue (selon les critères KDIGO soit une augmentation $>26,5 \mathrm{micromol} / \mathrm{L}$ en $48 \mathrm{~h}$ ou 1,5 à 1,9 fois le taux de base en moins de 7 jours), ainsi que des examens plus spécifiques et non réalisés systématiquement (AAN, ANCA, anticorps anti thyrö̈de, dosage des IgG4 sériques). 
- Les données d'imagerie : type d'imagerie réalisé (échographie, TDM, IRM, PET-TDM), la présence d'une prise de contraste après injection de produit de contraste ou d'une hyperfixation au PET-TDM, la présence d'adénopathies, la présence d'une dilatation pyélocalicielle (uni ou bilatérale), la localisation de la fibrose (aorte abdominale, thoracique, sus et/ou sous rénale), la taille des reins ou la présence d'une atrophie rénale. Enfin nous avons colligé lorsque c'était possible des éléments permettant d'orienter vers une cause primaire ou secondaire : extension en arrière de l'aorte, attraction ou refoulement des uretères, extension de la fibrose au-dessus des artères rénales jusqu'en dessous de la bifurcation iliaque

- Les données histologiques lorsqu'une biopsie a été réalisée et le rapport $\lg G 4 / \lg$ en immunohistochimie si disponible

- Les données concernant la prise en charge initiale : pose de sonde JJ (uni ou bilatérale) ou chirurgie urologique, nécessité d'une dialyse, le traitement médicamenteux (type, délai de traitement, si corticothérapie le délai de décroissance, la posologie à 6,12 et 18 mois, durée totale de traitement médical)

- La présence d'une rechute qui était définie comme la réapparition de signes cliniques évocateurs ou d'un syndrome inflammatoire biologique associée ou non à une majoration de la fibrose en imagerie alors que le patient était toujours traité par corticothérapie (= cortico-dépendance). Nous avons recueilli les données cliniques, biologiques, d'imagerie, le délai de rechute et la valeur de corticodépendance puis la prise en charge spécifique (nouvelle pose de sonde JJ, chirurgie, majoration de la corticothérapie, traitement de $2^{\text {ème }}$ ligne)

- La présence d'une récidive qui était définie comme la réapparition de signes cliniques évocateurs ou d'un syndrome inflammatoire biologique associée ou non à une majoration de la fibrose en imagerie alors que le patient n'était plus sous traitement médical. De même nous avons recueilli les données cliniques, biologiques, d'imagerie, le délai de récidive puis la prise en charge spécifique (nouvelle pose de sonde JJ, chirurgie, majoration de la corticothérapie, traitement de $2^{\text {ème }}$ ou $3^{\text {ème }}$ ligne)

- Les données concernant l'évolution : les complications liées aux sondes JJ, les complications et effets secondaire de la corticothérapie, la survenue d'une infection ayant nécessité une antibiothérapie au cours du traitement, la présence d'une rémission (définie comme l'absence de réapparition de signe clinique ou biologique après l'arrêt du traitement), la présence d'une insuffisance rénale chronique séquellaire et enfin l'ablation des sonde JJ ou la réalisation d'une chirurgie de dérivation des uretères. 
L'ensemble des données recueillies a permis de définir 2 groupes : fibroses rétropéritonéales idiopathiques et fibroses rétropéritonéales secondaires. Dans le groupe idiopathique, un sous groupe a été distingué en fonction de l'existence ou non d'une ectasie/anévrysme.

D. Analyses statistiques

Les variables continues sont exprimées en moyennes \pm écart-type si elles suivent une loi normale ou médianes [25-75 ${ }^{\text {ème }}$ percentile] dans le cas contraire. Les variables discrètes sont exprimées sous forme d'effectifs (pourcentage). Les variables continues ont été comparées par test de Mann Whitney ou t-test de Student lorsqu'applicable et les variables discrètes par test exact de Fisher ou test du Chi2 lorsqu'applicable. Les résultats étaient considérés significatifs pour un $p$ bilatéral inférieur à 0,05 . Toutes les analyses statistiques ont été réalisées avec le logiciel SPSS (version 23; SPSS Inc., Chicago, Illinois). Pour l'analyse des rechutes et récidives, le test de log Rank a été utilisé pour la comparaison avec les variables catégorielles et le modèle de Cox pour les variables continues. 


\section{Résultats}

Le recherche du mot clé " fibrose rétropéritonéale " sur la période 2010 - 2021 a permis de sélectionner 158 patients. Dans cette population, 102 ont été exclus :

- 69 patients pour lesquels le diagnostic de fibrose rétropéritonéale n'a pas été retenu après analyse du dossier médical :

- 24 néoplasies avec atteinte rétropéritonéale sans vraie FRP,

- 21 sténoses urétérales post radiques ou post chirurgicales,

- 1 sarcoïdose, 2 endométrioses, 4 diagnostics de reins chevelus isolés, 4 anévrysmes de l'aorte abdominales sans inflammation péri-anévrysmale, 1 maladie d'Erdheim Chester sans FRP, 1 cholangite sclérosante à IgG4 sans FRP, 1 aortite isolée, 1 pancréatite aiguë, 2 syndromes de jonction pyélo-urétérale et 7 patients pour lesquels il existait une suspicion clinique sans FRP confirmée en imagerie

- 15 patients atteints de FRP dont le suivi était assuré par un autre établissement

- 18 patients pour lesquels le nombre de données manquantes étaient trop important (perdus de vue, absence de suivi, FRP mentionnée comme antécédent)

Sur les 56 patients inclus, 33 avaient une FRP idiopathique. Au sein de ce dernier groupe, 13 patients avaient une ectasie ou un anévrysme de l'aorte. 


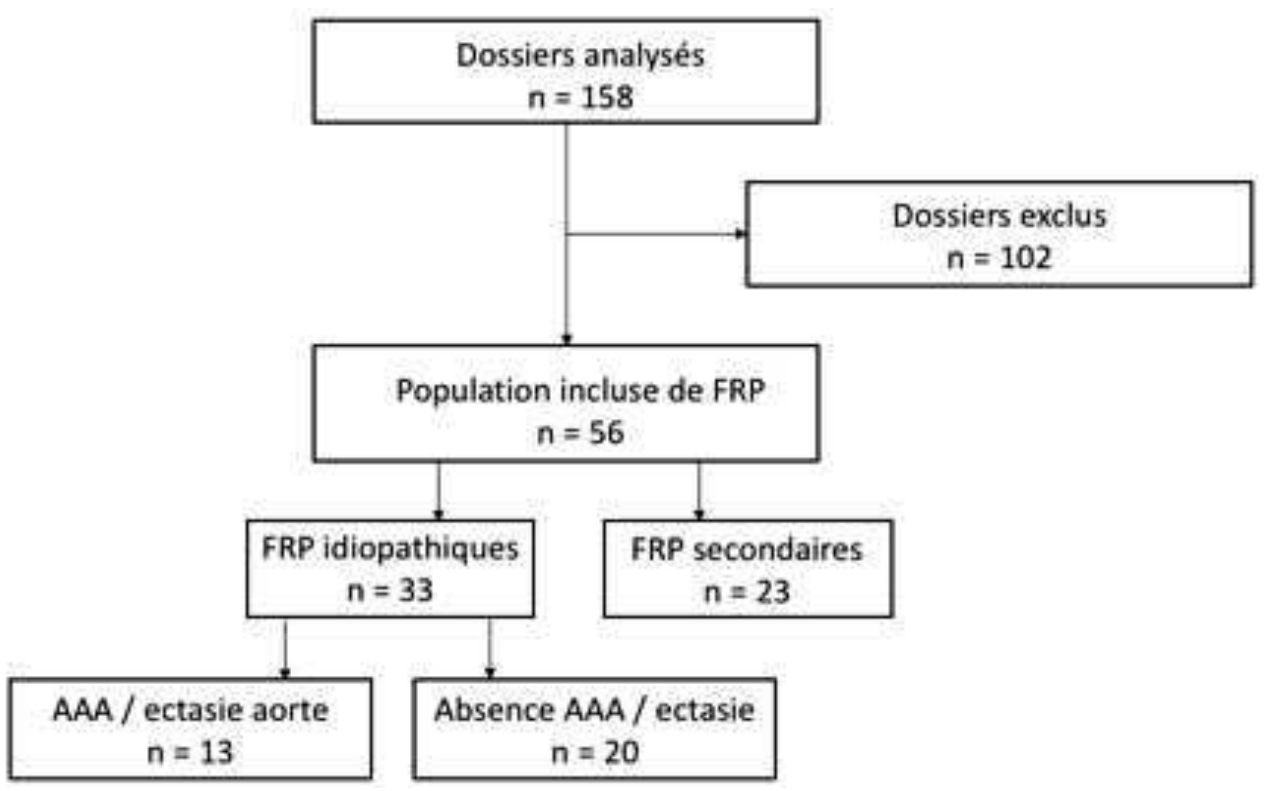

Figure 11 : Flow chart

\section{A. Caractéristiques de la population}

1. Population globale

Le sexe-ratio était de 2,7 hommes pour 1 femme ( 41 hommes soit 73,2\%), avec un âge moyen au diagnostic de 62,8 $\pm 12,6$ ans (extrêmes : 42 - 90 ans).

Une majorité de patients avait des facteurs de risque cardio-vasculaire (FDRCV) ( $n=49,89 \%)$. Par ordre décroissant : HTA (54,5\%), tabagisme actif $(48,2 \%)$, dyslipidémie $(41,8 \%)$, obésité (28\%) et diabète (27,3\%). L'exposition à l'amiante 5 des 24 patients pour lesquels l'information était disponible $(35,7 \%)$. Six patients avaient une pathologie inflammatoire : 1 spondylarthrite ankylosante, 1 maladie de Basedow, 1 psoriasis, 1 pemphigoïde bulleuse, 1 périartérite noueuse et 1 pseudopolyarthrite rhizomélique.

\section{Fibroses rétropéritonéales idiopathiques et secondaires}

Les seules différences entre les 2 types de fibroses concernaient l'obésité, plus fréquente chez les patients $\mathrm{FRPi}(41,4 \%$ vs $9,5 \%, \mathrm{p}=0,024)$, les antécédents de radio-chimiothérapie ou néoplasies plus fréquentes dans le groupe FRP secondaire $(47,8 \%$ vs $9,1 \%, p=0,02)$.

Concernant l'analyse de l'aorte, 8 patients dans le groupe FRP idiopathique étaient porteurs d'un anévrysme de l'aorte abdominale $(24,2 \%$ vs $4,5 \%)$ et 5 d'une ectasie de l'aorte abdominale $(15,2 \%$ vs $4,5 \%)$ 
L'exposition à l'amiante concernait $35,7 \%$ des patients avec FRPi $(n=5 / 24)$ et aucun avec FRP secondaire $(p=0,053)$.

Enfin, l'ensemble des pathologies inflammatoires recensées était observé dans le groupe FRP idiopathique $(p=0,030)$.

Les principales caractéristiques de la population sont détaillées dans le tableau 4.

\begin{tabular}{|c|c|c|c|c|}
\hline & $\begin{array}{l}\text { Population globale } \\
\qquad(n=56)\end{array}$ & $\begin{array}{l}\text { FRP } \\
\text { Idiopathique }(n=33)\end{array}$ & $\begin{array}{c}\text { FRP } \\
\text { secondaire }(n=23)\end{array}$ & $p$ \\
\hline \multicolumn{5}{|l|}{ Sexe (\%) } \\
\hline Homme & $41(73,2 \%)$ & $27(81,8 \%)$ & $14(60,9 \%)$ & \multirow[t]{2}{*}{0,125} \\
\hline Femme & $15(26,8 \%)$ & $6(18,2 \%)$ & $9(39,1 \%)$ & \\
\hline Âge au diagnostic & $62,8+/-12,6$ & $60,6[52,4-66,2]$ & $66,4[47,1-75,4]$ & 0,191 \\
\hline Facteurs de risque cardio-vasculaire & $49(89,1 \%)$ & $31(93,9 \%)$ & $18(81,8 \%)$ & 0,204 \\
\hline HTA & $30(54,5 \%)$ & $18(54,5 \%)$ & $12(54,5 \%)$ & 1 \\
\hline Diabète & $15(27,3 \%)$ & $12(36,4 \%)$ & $3(13,6 \%)$ & 0,074 \\
\hline Tabac actif $(n=47)$ & $23(48,9 \%)$ & $17(54,8 \%)$ & $6(37,5 \%)$ & 0,468 \\
\hline Dyslipidémie & $23(41,8 \%)$ & $16(48,5 \%)$ & $23(31,8 \%)$ & 0,271 \\
\hline Obésité (IMC > 30) ( $n=50)$ & $14(28 \%)$ & $12(41,4 \%)$ & $2(9,5 \%)$ & 0,024 \\
\hline IMC & $26,7+/-5,5$ & $29[23,9-32,2]$ & $23,9[21,9-25,6]$ & 0,007 \\
\hline Exposition à l'amiante ( $n=24)$ & $5(20,8 \%)$ & $5(35,7 \%)$ & 0 & 0,053 \\
\hline Atteinte vasculaire ischémique & $21(38,2 \%)$ & $12(36,4 \%)$ & $9(40,9 \%)$ & 0,782 \\
\hline Anévrysme aorte abdominale & $9(16,4 \%)$ & $8(24,2 \%)$ & $1(4,5 \%)$ & 0,070 \\
\hline Ectasie aorte abdominale & $6(10,9 \%)$ & $5(15,2 \%)$ & $1(4,5 \%)$ & 0,384 \\
\hline Chirurgie abdominale & $15(27,3 \%)$ & $9(27,3 \%)$ & $6(27,3 \%)$ & 1 \\
\hline $\begin{array}{l}\text { Antécédent de néoplasie ou radio- } \\
\text { chimiothérapie }\end{array}$ & $14(25 \%)$ & $3(9,1 \%)$ & $11(47,8 \%)$ & 0,002 \\
\hline Pathologies classiques associées & $6(12,5 \%)$ & $6(21,2 \%)$ & 0 & 0,030 \\
\hline Spondylarthrite ankylosante & 1 & 1 & 0 & - \\
\hline Vascularite & 1 & 1 & 0 & - \\
\hline Thyroïdite auto-immune & 1 & 1 & 0 & - \\
\hline Psoriasis & 1 & 1 & 0 & - \\
\hline Autre & 2 & 2 & 0 & - \\
\hline
\end{tabular}


B. Étiologies

Parmi les 56 diagnostics de fibrose rétropéritonéale, il y avait 33 FRP idiopathiques et 23 FRP secondaires. Une maladie associée aux IgG4 était recherchée chez 20 des 33 patients avec FRPi.

\begin{tabular}{|c|c|}
\hline FRP idiopathique & $33(58,9 \%)$ \\
\hline Avec recherche de MAG4 & $20(35,7 \%)$ \\
\hline Sans recherche de MAG4 & $13(23,2 \%)$ \\
\hline FRP secondaire & $23(41,1 \%)$ \\
\hline
\end{tabular}

La figure 12 présente les différentes étiologies des FRP secondaires de nos patients.

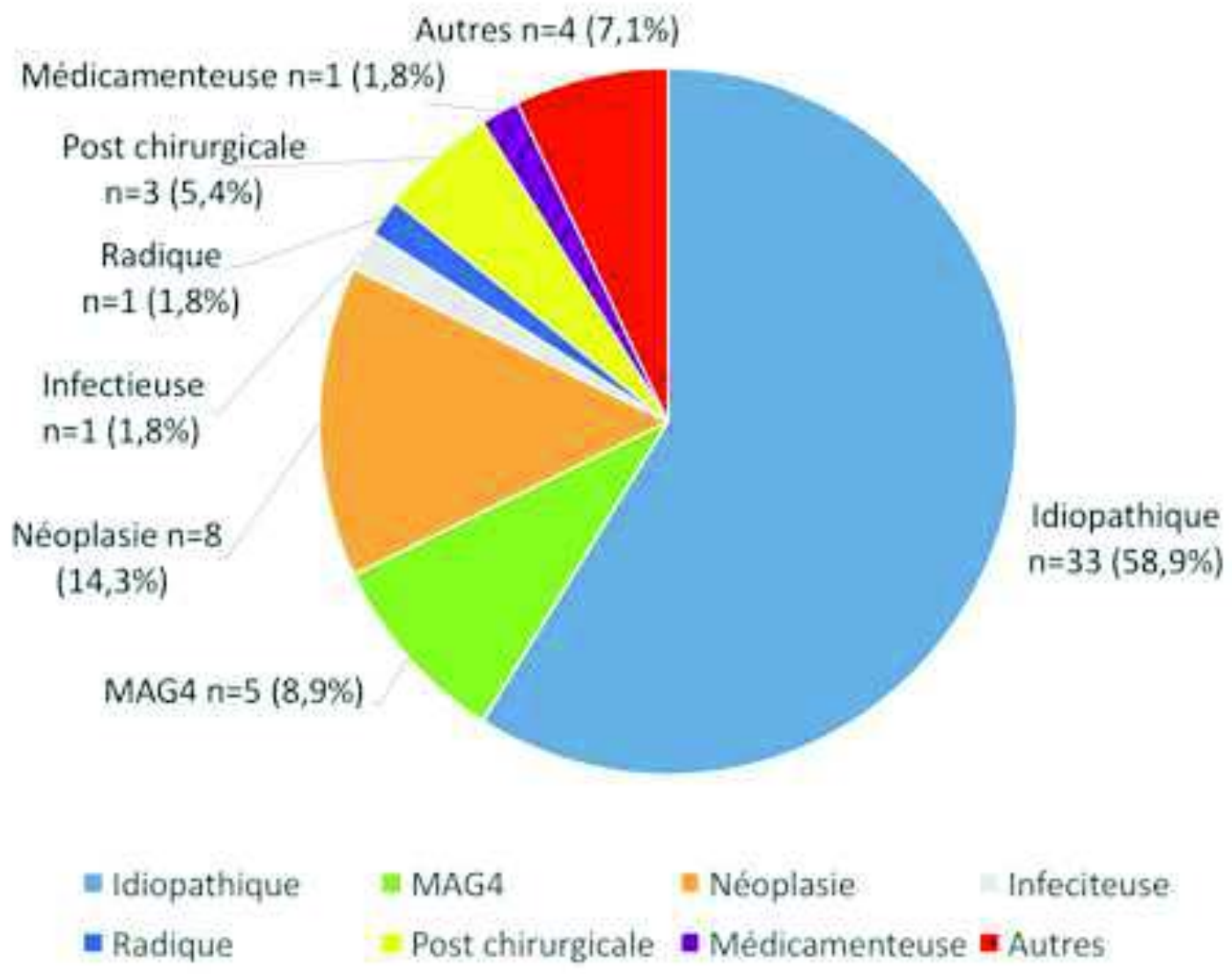

Figure 12 : Étiologies des FRP 
La principale cause de FRP secondaire retrouvée dans notre cohorte était le cancer pour 8 patients dont un carcinome mammaire, deux carcinomes cervico-utérins, un lymphome folliculaire, un carcinome épidermoïde bronchique, un carcinome urothélial de vessie, un carcinome rénal et un carcinome urothélial non étiqueté.

Sur les 5 patients recensés en tant que FRP secondaire à une maladie associée aux IgG4, 4 patients étaient classés en MAG4 possible et 1 patient en MAG4 certaine.

Parmi les 4 patients ayant une étiologie " autres ", 1 patiente avait une maladie auto-inflammatoire non classée, 1 tuberculose maladie était fortement suspectée chez un patient sans preuve bactériologique et 2 patients avaient une FRP secondaire à une maladie d'Erdheim-Chester.

Un seul patient avait une FRP médicamenteuse au Desernil ${ }^{\circledR}$ (anti-migraineux à base de méthysergide).

Parmi les 3 FRP post chirurgicales, une était survenue après une chirurgie de disque vertébral, une autre dans les suites d'un pontage aorto-bifémoral et enfin la dernière dans les suites d'une chirurgie d'anévrysme de l'aorte abdominale.

Enfin, la FRP post radique était rapportée chez un patient porteur d'un séminome testiculaire traité par chirurgie puis radio-chimiothérapie.

Une preuve histologique était disponible pour 9 des 56 patients (16,1\%). L'analyse histologique retrouvait pour 8 patients des remaniements fibreux inflammatoires non spécifiques avec un infiltrat Iymphocytaire plus ou moins dense, sans signe de malignité. Un patient présentait une prolifération tumorale lymphoïde d'architecture nodulaire avec un diagnostic de lymphome folliculaire.

Pour le patient avec la MAG4 certaine, la preuve histologique avait été obtenue sur une pièce de sigmoïdectomie retrouvant un infiltrat lymphoplasmocytaire, avec un rapport IgG4/IgG $>40 \%$. Le tableau clinique associait une fibrose rétropéritonéale à un nodule para-sigmoïdien gauche. 


\section{Présentation clinique}

Le symptôme inaugural le plus fréquent était une douleur abdominale et/ou lombaire retrouvée chez 75\% des patients. Des symptômes systémiques étaient observés chez 27 patients (48,2\%). Les autres symptômes, principalement secondaires à la compression de la fibrose sur les structures adjacentes étaient plus rarement observés : œdèmes des membres inférieurs dans 4 cas, claudication des membres inférieurs dans 6 cas, des symptômes de coliques néphrétiques dans 7 cas, des manifestations testiculaires chez 5 hommes et une dysurie pour 10 patients.

L'ensemble des données cliniques est détaillé dans le tableau 6.

La présentation clinique était globalement comparable dans le groupe FRP idiopathiques et FRP secondaires. La fièvre était plus souvent présente dans le groupe FRP secondaire $(p=0,001)$. La claudication des membres inférieurs était plus observée dans le groupe FRPi (6 patients) ( $p=0,037$ ). La présence d'une douleur abdominale au diagnostic était plus fréquente dans le groupe $\operatorname{FRPi}(p=0,029)$. Aucune manifestation testiculaire n'était observée dans le groupe FRP secondaire.

\begin{tabular}{|c|c|c|c|c|}
\hline & $\begin{array}{l}\text { Population globale } \\
\qquad(n=56)\end{array}$ & $\begin{array}{l}\text { FRP idiopathique } \\
\qquad(n=33)\end{array}$ & $\begin{array}{l}\text { FRP secondaire } \\
\qquad(n=23)\end{array}$ & $p$ \\
\hline Symptômes systémiques & $27(48,2 \%)$ & $13(39,4 \%)$ & $14(60,9 \%)$ & 0,174 \\
\hline AEG & $13(23,2 \%)$ & $7(21,2 \%)$ & $6(26 \%)$ & 0,768 \\
\hline Fébricule/fièvre & $7(12,5 \%)$ & $2(6 \%)$ & $5(21,7 \%)$ & 0,001 \\
\hline AEG + fébricule/fièvre & $7(12,5 \%)$ & $4(12,1 \%)$ & $3(13 \%)$ & 0,696 \\
\hline Douleurs & $43(75 \%)$ & $28(84,8 \%)$ & $14(60,9 \%)$ & 0,061 \\
\hline Lombalgies & $29(51,8 \%)$ & $20(60,6 \%)$ & $9(39,1 \%)$ & 0,174 \\
\hline Douleurs abdominales & $25(44,6 \%)$ & $19(57,6 \%)$ & $6(26,1 \%)$ & 0,029 \\
\hline Colique néphrétique & $7(12,5 \%)$ & $5(15,2 \%)$ & $2(8,7 \%)$ & 0,688 \\
\hline Dysurie & $10(17,9 \%)$ & $7(21,2 \%)$ & $3(13 \%)$ & 0,500 \\
\hline Manifestations testiculaires & $5(12,2 \%)$ & $5(15,2 \%)$ & 0 & 0,071 \\
\hline Douleurs & $3(7,3 \%)$ & $3(9 \%)$ & 0 & 0,079 \\
\hline Varicocèle ou hydrocèle & $2(4,9 \%)$ & $2(8,6 \%)$ & 0 & 0,153 \\
\hline Oedèmes des membres inférieurs & $4(7,1 \%)$ & $3(9,1 \%)$ & $1(4,3 \%)$ & 0,636 \\
\hline Claudication des membres inférieurs & $6(10,7 \%)$ & $6(18,2 \%)$ & 0 & 0,037 \\
\hline
\end{tabular}




\section{Présentation biologique}

Le tableau 7 présente les principales caractéristiques biologiques des patients au diagnostic. Un syndrome inflammatoire était présent dans $89,4 \%$ des cas. L'insuffisance rénale aigue concernait près de $48 \%$ des patients avec un taux de créatinine moyen de 190 10 mol/L. Le taux médian d'hémoglobine était significativement plus bas dans le groupe FRP secondaire $(11,2 \mathrm{~g} / \mathrm{dL})$ que dans le groupe FRPi $(12,45 \mathrm{~g} / \mathrm{dL})$ $(p=0,030)$, différence cliniquement peu significative.

La recherche systématique d'anticorps anti-nucléaires réalisée chez 27 patients n'était positive à titre significatif que pour 2 patients, parmi lesquels aucun n'avait de lupus systémique avéré. Ces deux patients avaient une FRP secondaire. La recherche d'ANCA et d'anticorps anti-thyroïdiens était négative chaque fois qu'elle était réalisée (respectivement pour 24 et 6 patients).

Le dosage pondéral des IgG4 réalisé pour 27 patients était pathologique (>1,35g/L) pour 4 patients. L'un des patients chez qui était porté le diagnostic de MAG4 avait un dosage d'IgG4 à $0,20 \mathrm{~g} / \mathrm{L}$ et le diagnostic était retenu devant une présentation clinique évocatrice associant une aortite thoracique, une atteinte pancréatique et une fibrose rétropéritonéale. Un taux élevé d'IgG4 était significativement plus observé dans le groupe FRP secondaire, en effet tous les patients du groupe FRP idiopathiques avaient un taux normal $d^{\prime} \operatorname{lgG} 4(p=0,002)$. Le taux médian était plus élevé dans le groupe FRP secondaire à $1,4 \mathrm{~g} / \mathrm{L}$ que dans le taux FRP idiopathique à $0,36 \mathrm{~g} / \mathrm{L}$.

\begin{tabular}{|c|c|c|c|c|}
\hline & $\begin{array}{l}\text { Population globale } \\
\qquad(n=56)\end{array}$ & $\begin{array}{l}\text { FRP idiopathique } \\
\qquad(n=33)\end{array}$ & $\begin{array}{l}\text { FRP secondaire } \\
\qquad(n=23)\end{array}$ & $p$ \\
\hline Anémie ( $\mathrm{F}: \mathrm{Hb}<12 \mathrm{~g} / \mathrm{dL}, \mathrm{H}: \mathrm{Hb}<13 \mathrm{~g} / \mathrm{dL})$ & $32(28,9 \%)$ & $16(61,5 \%)$ & $16(84,2 \%)$ & 0,182 \\
\hline Hémoglobine $(\mathrm{g} / \mathrm{dL})$ & $11,66+/-1,99$ & $12,45[11,4-13,7]$ & $11,2[10-12,3]$ & 0,030 \\
\hline Plaquettes (G/L) & $302,5(114,6)$ & $304[230,5-343]$ & $310[201-353]$ & 0,586 \\
\hline Syndrome inflammatoire biologique & $42(89,4 \%)$ & $27(93,1 \%)$ & $15(83,3 \%)$ & 0,357 \\
\hline $\mathrm{CRP}$ (mg/L) & $49,55+/-50,64$ & $30[16-55]$ & $49,5[11,75-98,25]$ & 0,256 \\
\hline Fibrinogène $(\mathrm{g} / \mathrm{L})$ & $5,8+/-1,5$ & $6[5,05-6,9]$ & $5,3[4,18-7,45]$ & 0,665 \\
\hline Insuffisance rénale aigue & $23(47,9 \%)$ & $12(44,4 \%)$ & $11(52,4 \%)$ & 0,771 \\
\hline Créatinine $(\mu \mathrm{mol} / \mathrm{L})$ & $189,5+/-180,8$ & $96[69-370]$ & $105[73,25-180,75]$ & 0,990 \\
\hline Présence d'anticorps anti-nucléaire $(n=27)$ & $2(7,4 \%)$ & 0 & $2(28,6 \%)$ & 0,060 \\
\hline Présence d'ANCA $(n=24)$ & 0 & 0 & 0 & \\
\hline Présence d'anticorps anti-thyroïdiens $(n=6)$ & 0 & 0 & 0 & \\
\hline Elévation taux IgG4 $(n=27)$ & $4(14,8 \%)$ & 0 & $4(57,1 \%)$ & 0,002 \\
\hline Valeurs des IgG4 (g/L) & $0,87+/-1,59$ & $0,36[0,15-0,71]$ & $1,4[0,028-3,474]$ & 0,407 \\
\hline
\end{tabular}




\section{E. Présentation radiologique}

Le tableau 8 résume les différentes caractéristiques radiologiques au diagnostic.

Une échographie abdominale était disponible pour 14 patients, une TDM pour 55 patients, une IRM pour 6 et un PET TDM pour 26 patients.

Dans 3 cas, la fibrose touchait l'aorte thoracique ainsi que l'aorte abdominale. Elle se limitait à la portion sous rénale pour les 53 autres patients. Une extension sus rénale était observée dans seulement 4 cas. L'atteinte des artères iliaques était fréquente (32 patients, 57,1\%).

La fibrose s'étendait de la portion sus rénale jusqu'à la bifurcation des artères iliaques pour 3 patients : 2 cas de FRP idiopathique et 1 cas de FRP secondaire.

L'information sur le rehaussement de la fibrose après injection de produit de contraste en scanner ou IRM n'était disponible que pour 10 patients. Elle était présente dans 8 cas sur 10 de FRPi $(p=0,001)$. Le réhaussement n'était pas associé à la rechute, la récidive ou la rémission.

La présence d'adénopathies significatives étaient décrites pour 17 patients, avec une fréquence plus élevée dans le groupe FRP secondaire $(p=0,035)$.

Une attraction médiale des uretères était observée pour $7 / 24$ patients (29\%) chez lesquels elle était recherchée, elle concernait 5 patients atteints de FRPi et 2 patients atteints de FRP secondaires. Un refoulement latéral n'était mentionné que chez 1 seul patient, atteint de FRP idiopathique.

Une dilatation des cavités pyélocalicielles était présente dans 40 cas, bilatérale pour 19 patients. Ces derniers patients avaient une insuffisance rénale aigue dans $94 \%$ des cas. Une atrophie rénale était observée au diagnostic chez 5 patients. Ces données concernant les complications urologiques étaient comparables dans les 2 groupes.

Un PET scanner au diagnostic était disponible pour 26 patients, avec une hyperfixation pour 23 patients $(82,1 \%)$ dont 15 dans le groupe $\mathrm{FRPi}$, sans différence significative entre les groupes. Cette hyperfixation n'était pas associée à la rechute, la récidive ou la rémission. 


\begin{tabular}{|c|c|c|c|c|}
\hline & $\begin{array}{l}\text { Population globale } \\
\qquad(n=56)\end{array}$ & $\begin{array}{l}\text { FRP idiopathique } \\
\qquad(n=33)\end{array}$ & $\begin{array}{l}\text { FRP secondaire } \\
\qquad(n=23)\end{array}$ & $p$ \\
\hline \multicolumn{5}{|l|}{ Localisation : } \\
\hline Atteinte de l'aorte thoracique & $3(5,4 \%)$ & $2(6,1 \%)$ & $1(4,3 \%)$ & 1 \\
\hline Atteinte de l'aorte abdominale & $52(92,9 \%)$ & $32(97 \%)$ & $20(87 \%)$ & 0,295 \\
\hline Atteinte de l'aorte sus rénale & $4(7,1 \%)$ & $3(9,1 \%)$ & $1(4,3 \%)$ & 0,636 \\
\hline Atteinte des artères iliaques & $32(57,1 \%)$ & $20(60,6 \%)$ & $12(52,2 \%)$ & 0,590 \\
\hline \multicolumn{5}{|l|}{ Complications rénales: } \\
\hline Dilatation unilatérale CPC & $21(37,5 \%)$ & $14(42,4 \%)$ & $7(30,4 \%)$ & 0,412 \\
\hline Dilatation bilatérale CPC & $19(33,9 \%)$ & $10(30,3 \%)$ & $9(39,1 \%)$ & 0,572 \\
\hline Présence d'une atrophie rénale & $5(9,1 \%)$ & $4(12,5 \%)$ & $1(4,3 \%)$ & 0,387 \\
\hline Présence d'adénopathies & $17(34,7 \%)$ & $6(21,4 \%)$ & $11(52,4 \%)$ & 0,035 \\
\hline $\begin{array}{l}\text { Rehaussement après injection } \\
\text { PDC (TDM/IRM) } n=10\end{array}$ & $8(80 \%)$ & $8(24 \%)$ & 0 & 0,001 \\
\hline $\begin{array}{l}\text { Hyperfixation pathologique (PET } \\
\text { TDM) } n=26\end{array}$ & $23(82,1 \%)$ & $15(88,2 \%)$ & $7(70 \%)$ & 0,326 \\
\hline
\end{tabular}

\section{F. Prise en charge initiale}

Le délai moyen de prise en charge médicale et/ou chirurgicale était de 2,1 mois, variant de 0 à 36 mois pour l'ensemble de la population. Le délai médian était de 1 mois pour les FRP idiopathique.

Concernant le drainage des voies urinaires, 35 patients (62,5\%) bénéficiaient d'une sonde double J (bilatérales pour 16 patients), sans différence entre les 2 groupes.

Neuf patients nécessitaient un autre type de chirurgie : une urétéro-iléoplastie, quatre néphrostomies, deux prothèses aorto-bifémorales, une urétérolyse avec intra péritonisation des uretères, une néphrectomie, une mise à plat avec greffe aorto-aortique chez un patient porteur d'un anévrysme de l'aorte abdominale avec fibrose péri-anévrysmale.

Trois patients nécessitaient une dialyse en urgence.

Concernant la prise en charge médicale, 38 patients $(69,1 \%)$ bénéficiaient d'un traitement médicamenteux, principalement une corticothérapie $(n=35)$. Les 3 autres patients relevaient d'une prise en charge spécifique : chimiothérapie (carcinome mammaire, lymphome folliculaire) et vemurafenib (maladie d'Erdheim Chester). Un seul patient était traité par bithérapie corticoïdes + tamoxifène. La corticothérapie était plus fréquemment initiée en cas de FRPi $(81,3 \%$ vs 39\%, $p=0,002)$, avec une posologie moyenne de $0,89 \mathrm{mg} / \mathrm{kg}$ et un délai de décroissance de 5 semaines. La durée moyenne de traitement était de 15,9 mois. Les données concernant les posologies, délais de décroissance et durée de la corticothérapie étaient comparable pour les FRPi et les FRP secondaires. 


\begin{tabular}{|c|c|c|c|c|}
\hline & $\begin{array}{l}\text { Population globale } \\
\qquad(n=56)\end{array}$ & $\begin{array}{l}\text { FRP idiopathique } \\
\qquad(n=33)\end{array}$ & $\begin{array}{l}\text { FRP secondaire } \\
\qquad(n=23)\end{array}$ & $p$ \\
\hline Délai de prise en charge (mois) & $2,1+/-5,3$ & $1[0-2]$ & $0[0-3]$ & 0,584 \\
\hline Pose de sonde JJ & $35(62,5 \%)$ & $20(60,6 \%)$ & $15(65,2 \%)$ & 0,785 \\
\hline Pose de sonde JJ bilatérale & $16(28,6 \%)$ & $8(24,2 \%)$ & $8(34,8 \%)$ & 0,549 \\
\hline Chirurgie autre & $9(16,1 \%)$ & $5(15,2 \%)$ & $4(17,4 \%)$ & 1 \\
\hline Dialyse & $3(5,4 \%)$ & $2(6,1 \%)$ & $1(4,3 \%)$ & 1 \\
\hline Traitement médicamenteux & $38(69,1 \%)$ & $26(81,3 \%)$ & $12(52,2 \%)$ & 0,037 \\
\hline Corticothérapie orale & $35(63,6 \%)$ & $26(81,6 \%)$ & $9(39,1 \%)$ & 0,002 \\
\hline Posologie d'attaque $(\mathrm{mg} / \mathrm{kg})$ & $0,89+/-0,16$ & $1[0,75-1]$ & $1[0,78-1]$ & 0,744 \\
\hline Posologie d'attaque (mg) & $65,45+/-16,07$ & $70[60-80]$ & $55[42,5-75]$ & 0,082 \\
\hline Délais de décroissance (semaines) & $5+/-2,5$ & $4[4-6,5]$ & $4[3-9]$ & 0,876 \\
\hline Posologie à 6 mois (mg) $n=29$ & $12,5[10-20]$ & $12,5[10-20]$ & $12,5[10-25]$ & 0,938 \\
\hline Posologie à 12 mois $(\mathrm{mg}) \mathrm{n}=22$ & $8[5-10]$ & $8[5,75-10]$ & $6[1,25-7,75]$ & 0,108 \\
\hline Posologie à 18 mois $(\mathrm{mg}) \mathrm{n}=10$ & $5,5[2,25-7,125]$ & $6[4-7,25]$ & 0 & 0,160 \\
\hline Durée de corticothérapie (mois) $n=13$ & $15,9+/-4,2$ & $15[11,75-19,25]$ & $17[15]$ & 0,548 \\
\hline
\end{tabular}

\section{G. Rechutes}

La rechute était définie par la réapparition de symptômes, de signes cliniques ou de signes radiologiques chez un patient encore traité par corticoïdes. La rechute survenait chez 9 patients (30\%) : 8 dans le groupe FRPi et 1 dans le groupe FRP secondaires, sans différence significative (log Rank $p=0.896$ ). Parmi ces 9 patients, 5 avaient une rechute clinique (douleurs lombaires, œdèmes des membres inférieurs, dysurie) et 6 avaient un syndrome inflammatoire biologique. Une majoration de la fibrose en imagerie était observée pour 4 patients.

Le délai médian de rechute était de 8 mois, avec une moyenne de corticothérapie à $10 \mathrm{mg}$.

Il n'existait pas de différence significative sur le risque de survenue d'une rechute selon le délai de décroissance après une phase d'attaque de corticoïdes (modèle de Cox - $p=0.195$ ). En cas de traitement d'attaque inférieur à 4 semaines, la récidive survenait dans 30\% des cas versus $18 \%$ ( $\log \operatorname{Rank} p=0.708$ ).

Concernant la prise en charge thérapeutique, une majoration de la corticothérapie était effectuée pour 5 patients, tous dans le groupe FRP idiopathique. Un traitement immunosuppresseur était instauré chez 7 patients : 6 porteurs de FRPi et 1 patient du groupe FRP secondaire pour lequel un traitement par azathioprine a été introduit, il s'agissait du patient atteint de la maladie auto-inflammatoire non classée. Pour 
les FRPi, les traitements de seconde ligne étaient variables : tamoxifène $(n=1)$, mycophenolate mofetil $(n=2)$, methotrexate $(n=4)$ et tocilizumab $(n=1)$.

Aucun patient ne nécessitait une nouvelle pose de sonde JJ ou une prise en charge chirurgicale lors de sa rechute.

Le tableau 10 détaille l'ensemble des données de rechute.

\begin{tabular}{|c|c|c|c|c|}
\hline & $\begin{array}{c}\text { Population } \\
\text { globale }(n=30)\end{array}$ & $\begin{array}{l}\text { FRP idiopathique } \\
\qquad(n=23)\end{array}$ & $\begin{array}{l}\text { FRP secondaire } \\
\qquad(n=7)\end{array}$ & $\boldsymbol{p}$ \\
\hline Survenue d'une rechute & $9(30 \%)$ & $8(34,8 \%)$ & $1(14,3 \%)$ & 0,896 \\
\hline Rechute clinique & $5(16,7 \%)$ & $4(17,4 \%)$ & $1(14,3 \%)$ & - \\
\hline Rechute biologique & $6(19,4 \%)$ & $5(20,8 \%)$ & $1(14,3 \%)$ & - \\
\hline Valeur CRP lors de la rechute (mg/L) & $50[7,75-62,5]$ & $51[45,25-67]$ & 66 & 0,373 \\
\hline Rechute radiologique & $4(13,3 \%)$ & $4(17,4 \%)$ & 0 & 0,548 \\
\hline Délai de rechute (mois) & $8[4-13,5]$ & $9[4,75-14,25]$ & 4 & 0,444 \\
\hline Valeur de corticodépendance (mg) & $10[7-20]$ & $10[6-17,5]$ & 20 & 0,444 \\
\hline \multicolumn{5}{|l|}{ Traitement } \\
\hline Majoration corticothérapie & $5(16,1 \%)$ & $5(20,8 \%)$ & 0 & 0,562 \\
\hline Introduction traitement immunosuppresseur & $7(22,6 \%)$ & $6(25 \%)$ & $1(14,3 \%)$ & 1 \\
\hline Tamoxifène & 1 & 1 & 0 & - \\
\hline Cellcept & 2 & 2 & 0 & - \\
\hline Methotrexate & 4 & 4 & 0 & - \\
\hline Azathioprine & 1 & 0 & 1 & - \\
\hline Tocilizumab & 1 & 1 & 0 & - \\
\hline Rituximab & 0 & 0 & 0 & - \\
\hline Nouvelle pose de JJ & 0 & 0 & 0 & - \\
\hline
\end{tabular}

\section{H. Récidives}

Une récidive survenait chez 5 patients (16\%), 3 dans le groupe FRPi et 2 dans le groupe FRP secondaire (2 MAG4). Parmi ces 5 patients, 4 avaient une récidive clinique (douleurs abdominales et lombaires), 3 avaient une récidive biologique et les 5 patients avaient une récidive à l'imagerie.

Le délai médian de récidive était de 12 mois après l'arrêt du traitement. La durée du traitement reçu initialement n'influençait pas le risque de survenue d'une récidive $(p=0,656)$.

La prise en charge consistait pour les 5 patients à la reprise d'une corticothérapie systémique, associée pour 4 patients à un traitement immunosuppresseur. Les 3 patients du groupe FRPi bénéficiait respectivement d'un traitement par tamoxifène, mycophenolate mofetil et methotrexate. 
Le patient du groupe FRP secondaire était atteint d'une FRP liée à une MAG4. II avait reçu successivement un traitement par méthotrexate arrêté pour aphtose invalidante, puis par rituximab avec à J5 un syndrome coronarien aigu, puis du mycophenolate mofetil arrêté pour syphilis oculaire.

Une nouvelle pose de sonde JJ était nécessaire pour 2 patients du groupe FRP idiopathique.

Une biopsie était réalisée chez 1 patient du groupe FRPi dont l'analyse histologique confirmait le caractère idiopathique avec la mise en évidence d’un tissu scléreux aspécifique, sans signe de malignité.

\begin{tabular}{|c|c|c|c|c|}
\hline & $\begin{array}{c}\text { Population } \\
\text { globale }(n=31)\end{array}$ & $\begin{array}{l}\text { FRP idiopathique } \\
\qquad(n=24)\end{array}$ & $\begin{array}{l}\text { FRP secondaire } \\
\qquad(n=7)\end{array}$ & $p$ \\
\hline Survenue d'une récidive & $5(16,1 \%)$ & $3(12,5 \%)$ & $2(28,6 \%)$ & 0,562 \\
\hline Récidive clinique & $4(13,3 \%)$ & $3(12,5 \%)$ & $1(16,7 \%)$ & - \\
\hline Récidive biologique & $3(9,7 \%)$ & $2(8,3 \%)$ & $1(14,3 \%)$ & - \\
\hline Valeur CRP lors de la récidive & $40[32-N A]$ & $123[32-N A]$ & 40 & 1 \\
\hline Récidive radiologique & $5(16,1 \%)$ & $3(12,5 \%)$ & $2(28,6 \%)$ & 0,562 \\
\hline Délai de récidive (mois) & $12[3,5-22,5]$ & 12 [5-NA] & $16,5[2-\mathrm{NA}]$ & 0,800 \\
\hline \multicolumn{5}{|l|}{ Traitement } \\
\hline Reprise corticothérapie & $5(16,1 \%)$ & $3(12,5 \%)$ & $2(28,6 \%)$ & 0,562 \\
\hline $\begin{array}{l}\text { Introduction traitement } \\
\text { immunosuppresseur }\end{array}$ & $4(12,9 \%)$ & $3(12,5 \%)$ & $1(14,3 \%)$ & 1 \\
\hline Tamoxifène & 1 & 1 & 0 & - \\
\hline Cellcept & 2 & 1 & 1 & - \\
\hline Methotrexate & 2 & 1 & 1 & - \\
\hline Azathioprine & 0 & 0 & 0 & - \\
\hline Rituximab & 1 & 0 & 1 & - \\
\hline Nouvelle pose de JJ & $2(6,5 \%)$ & $2(8,3 \%)$ & 0 & 1 \\
\hline
\end{tabular}

\section{I. Évolution}

Sur les 35 patients ayant nécessité une pose de sonde JJ, 18 (51,4\%) avaient au cours du suivi une complication en lien direct avec ce matériel : hématurie macroscopique avec déglobulisation, douleurs lombaires invalidantes, insuffisance rénale aigue en lien avec une migration de la sonde. Enfin, pour 13 patients $(37,1 \%)$ il s'agissait de complications infectieuses : infections urinaires à répétition et pyélonéphrites obstructives, compliquées de choc septique pour 2 patients.

Concernant la tolérance de la corticothérapie, 20 patients (55,5\%) avaient des effets indésirables : survenue ou aggravation d'un diabète $(n=10)$, prise de poids importante $(n=10)$, trouble de l'humeur $(n=5)$, ostéopénie ( $n=2)$, hypertension artérielle $(n=3)$, glaucome $(n=1)$, hématomes cutanés $(n=1)$, acné $(n=1)$. Onze 
événements infectieux survenait au cours du suivi : 1 pneumopathie, 7 pyélonéphrites aigue, 2 épisodes de zona, 1 syphilis oculaire.

\begin{tabular}{|c|c|c|c|c|}
\hline & Population globale & FRP idiopathique & FRP secondaire & $p$ \\
\hline $\begin{array}{l}\text { Complications, effets secondaires liées aux } \\
\text { sondes JJ ( } n=35)\end{array}$ & $18 / 35(51,4 \%)$ & $9 / 20(45 \%)$ & $9 / 15(60 \%)$ & 0,394 \\
\hline Hématurie & $6(17,1 \%)$ & $3(15 \%)$ & $3(20 \%)$ & 0,697 \\
\hline Douleurs lombaires & $4(11,4 \%)$ & $2(10 \%)$ & $2(13,3 \%)$ & 0,759 \\
\hline Insuffisance rénale aigue & $4(11,4 \%)$ & $2(10 \%)$ & $2(13,3 \%)$ & 0,759 \\
\hline Complications infectieuses & $13(37,1 \%)$ & $6(30 \%)$ & $7(46,7 \%)$ & 0,312 \\
\hline $\begin{array}{l}\text { Complications, effets secondaires liées aux } \\
\text { corticoïdes }(n=36)\end{array}$ & $20 / 36(55,5 \%)$ & $15 / 27(55,5 \%)$ & $5 / 9(55,5 \%)$ & 0,090 \\
\hline $\begin{array}{l}\text { Diabète cortico-induit/déséquilibre de } \\
\text { diabète }\end{array}$ & $10(27,8 \%)$ & $9(33,3 \%)$ & $1(11,1 \%)$ & 0,197 \\
\hline Prise de poids importante/dyslipidémie & $10(27,8 \%)$ & $8(29,6 \%)$ & $2(22,2 \%)$ & 0,667 \\
\hline Trouble de l'humeur & $5(13,9 \%)$ & $5(18,5 \%)$ & 0 & 0,164 \\
\hline Ostéopénie & $2(5,6 \%)$ & $1(3,7 \%)$ & $1(11,1 \%)$ & 0,400 \\
\hline HTA & $3(8,3 \%)$ & $2(7,4 \%)$ & $1(11,1 \%)$ & 0,727 \\
\hline Complications infectieuses & $11(30,6 \%)$ & $8(29,6 \%)$ & $3(33,3 \%)$ & 0,834 \\
\hline Autres & $3(8 ? 3 \%)$ & $3(11,1 \%)$ & 0 & 0,296 \\
\hline
\end{tabular}

Le suivi moyen de la cohorte était de 59,8 $\pm 62,5$ mois. La rémission, définie comme l'absence de récidive clinique, biologique ou radiologique, était observée chez 26 patients dans la population globale (47,3\%), significativement plus élevé dans le groupe FRPi $(66,7 \%$ vs 18,2\%, p=0,001). Les 4 patients considérés en rémission de la population FRP secondaire concernait la FRP médicamenteuse, celle secondaire à une pathologie auto inflammatoire inclassée et 2 FRP dans un contexte de MAG4.

Sur les 35 patients ayant nécessité une dérivation urétérale, seuls 14 patients avaient bénéficié du retrait de ces sondes à la fin de notre recueil. Dans 3 cas, une chirurgie de dérivation des uretères était réalisée. II n'existait pas de différence significative entre nos 2 groupes, bien que 34,4\% des patients du groupe FRPi aient eu une ablation des sondes versus 13\% du groupe FRP secondaire. Parmi les 14 patients chez qui un retrait des sondes était réalisé, 9 (64,3\%) avaient bénéficié d’une corticothérapie.

Une insuffisance rénale chronique survenait pour 18 patients, avec un stade moyen de $3,3 \pm 1,2$. On notait une atteinte terminale chez $7 \%$ des patients. La survenue d'une insuffisance rénale chronique n'était pas significativement différente entre les groupes primitif ou secondaire. Tous les patients atteints $d^{\prime}$ 'insuffisance rénale chronique à la fin du suivi avaient au moins un facteur de risque cardio vasculaire au diagnostic $(p=0,161)$. La présence d'une hypertension artérielle au diagnostic était significativement associée au risque de survenue d'une insuffisance rénale chronique $(p=0,008)$. Ce n'était pas le cas pour les autres 
facteurs de risque cardiovasculaire (obésité, diabète, dyslipidémie, tabagisme actif). Une insuffisance rénale chronique survenait d'autant plus chez les patients ayant nécessité une pose de sondes JJ $(0,039)$.

\begin{tabular}{|c|c|c|c|c|}
\hline & $\begin{array}{c}\text { Population } \\
\text { globale }(n=56)\end{array}$ & $\begin{array}{l}\text { FRP idiopathique } \\
\qquad(n=33)\end{array}$ & $\begin{array}{l}\text { FRP secondaire } \\
\qquad(n=23)\end{array}$ & $p$ \\
\hline Rémission & $26(47,3 \%)$ & $22(66,7 \%)$ & $4(18,2 \%)$ & 0,001 \\
\hline Ablation des sondes JJ ( $n=35)$ & $14(40 \%)$ & $11(34,4 \%)$ & $3(13 \%)$ & 0,117 \\
\hline Chirurgie de dérivation des uretères & $3(5,4 \%)$ & $2(6,1 \%)$ & $1(4,3 \%)$ & 1 \\
\hline $\begin{array}{l}\text { Traitement médicamenteux en cours lors du } \\
\text { dernier suivi }\end{array}$ & $20(51,3 \%)$ & $13(39,4 \%)$ & $7(31,8 \%)$ & 0,775 \\
\hline Survenue d'une insuffisance rénale chronique & $18(32,1 \%)$ & $11(33,3 \%)$ & $7(30,4 \%)$ & 1 \\
\hline Stade 1 & 1 & 1 & 0 & - \\
\hline Stade 2 & 3 & 3 & 0 & - \\
\hline Stade 3 & 7 & 4 & 3 & - \\
\hline Stade 4 & 3 & 1 & 2 & - \\
\hline Stade 5 & 4 & 2 & 2 & - \\
\hline
\end{tabular}

\section{J. Population AAA et ectasie de l'aorte}

1. Caractéristiques globales

Parmi la population de fibrose rétropéritonéale idiopathique, les patients avec AAA ou ectasie de l'aorte abdominale ont été comparés à ceux n'en ayant pas, le tableau 14 résume les différentes caractéristiques globales de ces patients.

Il n'existait pas de différence significative entre ces deux populations en ce qui concerne le genre. L'âge médian au diagnostic était plus élevé dans le groupe AAA $(65,7$ vs $56,2, p=0,047)$. Concernant les facteurs de risque cardio-vasculaire, seule l'obésité était significativement plus fréquente chez les patients atteints de FRPi sans dilatation de l'aorte abdominale ( $8 \%$ vs $65 \%, p=0,003$ ).

Les antécédents de chirurgie abdominale, d'atteinte vasculaire ischémique, d'exposition à l'amiante et d'association avec des pathologies auto-immunes étaient comparables entre ces 2 populations. 


\begin{tabular}{|c|c|c|c|}
\hline & $\begin{array}{l}\text { FRPi avec AAA/ectasie } \\
\qquad(n=13)\end{array}$ & $\begin{array}{l}\text { FRPi sans AAA/ectasie } \\
\qquad(n=20)\end{array}$ & $p$ \\
\hline \multicolumn{4}{|l|}{ Sexe (\%) } \\
\hline Homme & $12(92,3 \%)$ & $15(75 \%)$ & \multirow[t]{2}{*}{0,364} \\
\hline Femme & $1(7,7 \%)$ & $5(25 \%)$ & \\
\hline Âge au diagnostic & $65,7[59,4-68,2]$ & $56,2[49,4-65,8]$ & 0,047 \\
\hline Facteurs de risque cardio-vasculaire & $13(100 \%)$ & $18(90 \%)$ & 0,508 \\
\hline HTA & $7(53,8 \%)$ & $11(55 \%)$ & 1 \\
\hline Diabète & $2(15,4 \%)$ & $10(50 \%)$ & 0,067 \\
\hline Tabac actif & $10(76,9 \%)$ & $7(38,9 \%)$ & 0,065 \\
\hline Dyslipidémie & $8(61,5 \%)$ & $8(40 \%)$ & 0,296 \\
\hline Obésité (IMC > 30) & $1(8,3 \%)$ & $11(64,7 \%)$ & 0,003 \\
\hline IMC & $25,3[21-28,3]$ & $30,5[28,7-34]$ & 0,005 \\
\hline Exposition à l'amiante $(n=14)$ & $4(57,1 \%)$ & $1(14,3 \%)$ & 0,266 \\
\hline Atteinte vasculaire ischémique & $7(53,8 \%)$ & $5(25 \%)$ & 0,142 \\
\hline Chirurgie abdominale & $4(30,8 \%)$ & $5(25 \%)$ & 1 \\
\hline $\begin{array}{l}\text { Antécédent de néoplasie ou radio- } \\
\text { chimiothérapie }\end{array}$ & $3(23,1 \%)$ & 0 & 0,052 \\
\hline Pathologies classiques associées & $2(15,4 \%)$ & $4(20 \%)$ & 1 \\
\hline
\end{tabular}

2. Données cliniques, biologiques, radiologiques et histologiques

Les données cliniques étaient comparables dans ces deux populations. Il en était de même pour les examens biologiques. On notait seulement une médiane de créatinine à $217 \mu \mathrm{mol} / \mathrm{L}$ dans le groupe AAA contre $72 \mu \mathrm{mol} / \mathrm{L}$ dans le groupe sans AAA, sans différence significative.

Les données d'imagerie étaient également comparables dans les deux groupes.

Sept biopsies étaient réalisées dans le groupe sans AAA ou ectasie, aucune dans le groupe avec AAA $(p=0,027)$.

Toutes ces données sont résumées dans le tableau 15. 
Tableau 15: Caractéristiques cliniques, biologiques, radiologiques et histologiques des patients FRPi avec et sans AAA/ectasie

FRPi avec AAA/ectasie FRPi sans AAA/ectasie $p$

( $n=13$ )

( $n=20$ )

Données cliniques :

Symptômes systémiques

$5(38,5 \%)$

$8(40 \%)$

1

Douleurs

$12(92,3 \%)$

$16(80 \%)$

0,625

Colique néphrétique

$2(15,4 \%)$

$3(15 \%)$

$1(7,7 \%)$

$6(30 \%)$

1

Dysurie

$1(8,3 \%)$

$4(26,6 \%)$

0,202

Manifestations testiculaires

0

$3(15 \%)$

Oedèmes des membres inférieurs

$3(23,1 \%)$

$3(15 \%)$

0,261

Claudication des membres inférieurs

Données biologiques :

Anémie

$9(75 \%)$

$7(50 \%)$

0,248

Hémoglobine $(\mathrm{g} / \mathrm{dL})$

$11,8[9,5-12,9]$

$12(92,3 \%)$

$43[26-58,5]$

0,498

CRP (mg/L)

$6(54,5 \%)$

$6(37,5 \%)$

0,226

Insuffisance rénale aigue

217 [96-370]

72 [69-100]

0,452

Créatinine $(\mu \mathrm{mol} / \mathrm{L})$

Données d'imagerie :

Atteinte de l'aorte thoracique

Atteinte de l'aorte abdominale

0

$12(92,3 \%)$

$1(7,7 \%)$

$7(53,8 \%)$

$5(38,5 \%)$

$2(10 \%) \quad 0 ; 508$

$20(100 \%) \quad 0,394$

$2(10 \%)$

$13(65 \%)$

0,717

$9(45 \%)$

1

$6(30 \%)$

$2(15,4 \%)$

$7(35 \%)$

1

1
$4(30,8 \%)$

$2(10,5 \%)$

0

Biopsie de la FRP 


\section{Prise en charge}

Les données sur la prise en charge étaient comparables dans les 2 groupes. Elles sont détaillées dans le tableau 16.

Huit patients nécessitaient la pose de sonde double J dans le groupe avec AAA contre 12 dans l'autre groupe. Les patients qui recevaient un traitement étaient tous traités par corticoïdes, avec des modalités d'administration comparables dans les 2 groupes (posologie, délai de décroissance, durée).

\begin{tabular}{|c|c|c|c|}
\hline & $\begin{array}{l}\text { FRPi avec AAA/ectasie } \\
\qquad(n=13)\end{array}$ & $\begin{array}{l}\text { FRPi sans AAA/ectasie } \\
\qquad(n=20)\end{array}$ & $p$ \\
\hline Pose de sonde JJ & $8(61,5 \%)$ & $12(60 \%)$ & 1 \\
\hline Pose de sonde JJ bilatérale & $3(23,1 \%)$ & $5(25 \%)$ & 1 \\
\hline Chirurgie autre & $2(15,4 \%)$ & $3(15 \%)$ & 1 \\
\hline Dialyse & $2(15,4 \%)$ & 0 & 0,148 \\
\hline Traitement médicamenteux & $12(92,3 \%)$ & $14(73,7 \%)$ & 0,361 \\
\hline Corticothérapie orale & $12(92,3 \%)$ & $14(73,7 \%)$ & 0,361 \\
\hline Posologie d'attaque (mg/kg) & $1[1-1]$ & $0,9[0,7-1]$ & 0,179 \\
\hline Posologie d'attaque (mg) & $70[60-80]$ & $72,5[60-80]$ & 0,695 \\
\hline Délais de décroissance (semaines) & $4[3-6]$ & $4,5[4-6]$ & 0,171 \\
\hline Durée de corticothérapie (mois) & $13,5[10,5-15]$ & $17[15-23]$ & 0,084 \\
\hline
\end{tabular}

\section{Rechutes et récidives}

Trois rechutes survenaient dans le groupe avec AAA et 5 dans le groupe sans AAA. Les délais médians de rechute étaient respectivement de 4 mois et 10 mois, sans différence significative. On notait une valeur de cortico-dépendance plus haute dans le groupe des anévrysmes/ectasie ( $20 \mathrm{mg}$ vs $9 \mathrm{mg}, p=0,047)$. Le reste des données concernant les rechutes étaient comparables dans les deux populations. 
Une seule récidive survenait dans le groupe AAA et 3 dans l'autre groupe. Les délais de récidives étaient respectivement de 12 mois et 5 mois. La reprise de la corticothérapie et l'ajout d'un traitement de seconde ligne étaient effectués dans $100 \%$ des cas dans les deux groupes. Le reste des données était comparable dans les deux populations.

Le tableau 17 résume l'ensemble des données de rechute et récidive dans ces 2 groupes.

\begin{tabular}{|c|c|c|c|}
\hline & $\begin{array}{l}\text { FRPi avec AAA/ectasie } \\
\qquad(n=13)\end{array}$ & $\begin{array}{l}\text { FRPi sans AAA/ectasie } \\
\qquad(\mathrm{n}=\mathbf{2 0})\end{array}$ & $p$ \\
\hline Survenue d'une rechute & $3(27,3 \%)$ & $5(27,8 \%)$ & 0,545 \\
\hline Rechute clinique & 0 & $4(80 \%)$ & - \\
\hline Rechute biologique & $3(100 \%)$ & $2(40 \%)$ & - \\
\hline Rechute radiologique & $1(33,3 \%)$ & $3(75 \%)$ & 0,486 \\
\hline Délai de rechute (mois) & $4[2,5-10,5]$ & $10[8-12]$ & 0,456 \\
\hline Valeur de corticodépendance (mg) & $20[15-30]$ & $9[5-10]$ & 0,047 \\
\hline Majoration corticothérapie & $2(66,7 \%)$ & $3(60 \%)$ & 1 \\
\hline Introduction traitement immunosuppresseur & $3(100 \%)$ & $3(60 \%)$ & 0,464 \\
\hline Nouvelle pose de JJ & 0 & 0 & - \\
\hline Survenue d'une récidive & $1(8,3 \%)$ & $3(16,7 \%)$ & 0,632 \\
\hline Récidive clinique & 0 & $2(66,7 \%)$ & - \\
\hline Récidive biologique & $1(100 \%)$ & $2(66,7 \%)$ & - \\
\hline Récidive radiologique & $1(100 \%)$ & $3(100 \%)$ & - \\
\hline Délai de récidive (mois) & 12 & $5[3-14,5]$ & - \\
\hline \multicolumn{4}{|l|}{ Valeur de corticodépendance ( $\mathrm{mg}$ ) } \\
\hline Reprise corticothérapie & $1(100 \%)$ & $3(100 \%)$ & - \\
\hline Introduction traitement immunosuppresseur & $1(100 \%)$ & $3(100 \%)$ & - \\
\hline Nouvelle pose de JJ & $1(100 \%)$ & $2(66,7 \%)$ & 1 \\
\hline
\end{tabular}




\section{5. Évolution}

Neuf patients du groupe AAA (69\%) étaient en rémission à la fin du suivi et 13 dans le groupe sans AAA (65\%). L'ablation des sondes doubles J était réalisée pour 5 patients du premier groupe et 6 du second. Une insuffisance rénale chronique survenait pour 6 patients du groupe AAA (46\%) dont 2 insuffisances rénales chroniques terminales, et 5 patients $(25 \%)$ du deuxième groupe, sans différence significative. Les taux de complications liées à la corticothérapie et aux sondes JJ étaient comparables dans les 2 groupes.

\begin{tabular}{|c|c|c|c|}
\hline & $\begin{array}{l}\text { FRPi avec AAA/ectasie } \\
\qquad(n=13)\end{array}$ & $\begin{array}{l}\text { FRPi sans AAA/ectasie } \\
\qquad(\mathrm{n}=\mathbf{2 0})\end{array}$ & $p$ \\
\hline Durée de suivi (mois) & $28,6[20,7-64,4]$ & $39,2[21-120]$ & 0,439 \\
\hline Rémission & $9(69,2 \%)$ & $13(65 \%)$ & 1 \\
\hline Ablation des sondes JJ & $5(38,5 \%)$ & $6(31,6 \%)$ & 0,721 \\
\hline Chirurgie de dérivation des uretères & 0 & $2(10 \%)$ & 0,508 \\
\hline $\begin{array}{l}\text { Traitement médicamenteux en cours lors du } \\
\text { dernier suivi }\end{array}$ & $6(46,2 \%)$ & $7(35 \%)$ & 0,717 \\
\hline Survenue d'une insuffisance rénale chronique & $6(46,2 \%)$ & $5(25 \%)$ & 0,270 \\
\hline Stade 1 & 1 & 0 & \multirow{5}{*}{0,777} \\
\hline Stade 2 & 2 & 1 & \\
\hline Stade 3 & 1 & 3 & \\
\hline Stade 4 & 0 & 1 & \\
\hline Stade 5 & 2 & 0 & \\
\hline $\begin{array}{l}\text { Complications, effets secondaires liées aux sondes } \\
\text { JJ }\end{array}$ & $2(15,4 \%)$ & $7(35 \%)$ & 0,263 \\
\hline $\begin{array}{l}\text { Complications, effets secondaires liées aux } \\
\text { corticoïdes }\end{array}$ & $4(33,3 \%)$ & $11(55 \%)$ & 0,291 \\
\hline
\end{tabular}




\section{Discussion}

\section{A. Critères d'inclusion des patients}

Le diagnostic de FRPi reste un diagnostic d'exclusion qui repose avant tout sur les données de l'imagerie. Une meilleure compréhension de la physiopathologie et l'identification de la MAG4 au sein des FRP sont des enjeux importants pour cette maladie rare. Enfin, la prise en charge thérapeutique n'est pas consensuelle et repose sur des recommandations d'experts, avec peu d'essais cliniques.

La FRPi s'inscrit désormais dans le cadre des péri-aortites chroniques, la distinguant des anévrysmes avec fibrose péri anévrysmale et des anévrysmes inflammatoires. Dans ce travail rétrospectif, le terme de fibrose rétropéritonéale a été retenu pour décrire les péri-aortites chroniques, incluant ainsi les 3 entités compte tenu d'une base physiopathologique commune supposée. Les aortites ont été exclu considérant qu'elles ne faisaient pas parties du même spectre de pathologie. Il faut néanmoins garder à l'esprit que certains auteurs suggèrent que les péri-aortites chroniques pourraient être considérées comme une vascularite des gros vaisseaux telles que l'artérite à cellules géantes, compte tenu de la présence d'une inflammation adventitielle intense et de l'atteinte de plusieurs segments vasculaires, en particulier l'aorte thoracique ${ }^{78,106}$.

Cette étude rétrospective monocentrique avait pour objectif de décrire les caractéristiques clinicobiologiques et radiologiques des patients atteints de fibrose rétropéritonéale suivies au sein des services de médecine interne, urologie et néphrologie. Le nombre de patients inclus $(n=56)$ se rapproche des séries françaises rapportées dans la littérature ${ }^{105,107}$, avec des caractéristiques comparables ${ }^{4,60,108}$.

\section{B. Épidémiologie}

Notre cohorte comportait une majorité d'hommes, dont l'âge se situait autour de $60 a^{4}{ }^{4}$. Cette prépondérance masculine semblait plus marquée dans la population de FRP idiopathique. Compte tenu du rôle pathogénique suggéré de l'athérosclérose dans la FRPi, cette différence pourrait être le résultat de l'augmentation de l'incidence de la maladie athéromateuse chez les hommes. De plus, cette différence tend à disparaître en ce qui concerne les FRP secondaires, notamment d'origine néoplasique ${ }^{109}$.

De nombreuses publications retrouvent un lien fort entre FRPi et facteurs de risque cardiovasculaire 4,5,10,110. Dans notre série, $89 \%$ de la population globale et $93,9 \%$ des patients FRPi avaient un terrain 
cardiovasculaire. La recherche de FDRCV paraît ainsi importante chez ces patients, ce d'autant qu'une grande partie sera traité par corticothérapie. L'obésité était présente chez plus de $40 \%$ des patients du groupe FRPi, suggérant encore une fois le lien entre FDRCV et FRP. De plus, la fréquence des formes néoplasiques dans la population FRP secondaire peut également expliquer cette disparité, les patients atteints d'une néoplasie présentant plus souvent un amaigrissement. Cette observation est proche des données de la littérature ${ }^{4,13}$, avec par exemple un IMC moyen de 26,6 dans la cohorte de Van Bommel et al. portant sur 53 patients atteints de fibroses rétropéritonéales idiopathiques ${ }^{57}$.

L'exposition à l'amiante est bien décrite comme étant associée au risque de $\mathrm{FRPi}^{3,13}$. Dans notre cohorte, l'association n'était pas significativement associée au caractère primitif de la FRP mais s'en approchait $(35,7 \%$ vs $0 \%, p=0,053)$, probablement en lien avec le faible effectif et le manque de données concernant ce critère. Un tabagisme actif était présent chez plus de $50 \%$ de notre population, ce qui concorde avec les données de la littérature ${ }^{13}$.

Au sein du groupe FRPi, 8 patients avaient une dilatation de l'aorte abdominale ( 4 anévrysmes inflammatoires et 4 anévrysmes avec fibrose péri anévrysmale). Cinq patients avaient une ectasie de l'aorte abdominale. Peu de données existent ce jour sur la fréquence des fibroses péri anévrysmales et des anévrysmes inflammatoires au sein des péri aortites chroniques. La comparaison de ces formes avec les FRPi non anévrysmale ne montrait pas de différence concernant les FDRCV en dehors de l'obésité ; il n'y avait pas non plus de différence d'évolution en termes de rechute ou de récidive. Néanmoins le faible effectif de la cohorte ne permet pas de conclure de façon robuste à l'absence de différence entre ces sous-groupes.

\section{Hypothèse de l'auto-immunité}

L'hypothèse selon lequel la FRPi a une origine auto-immune est renforcée par sa fréquente association avec des maladies auto-immunes, touchant d'autres organes ou structures. Dans notre population de FRPi, une pathologie inflammatoire était recensée chez 6 patients (et aucune parmi les FRP secondaires). Dans une étude portant sur une série de 16 patients atteints de péri-aortites chroniques, trois d'entre eux présentaient une vascularite à ANCA avec un tableau d'insuffisance rénale aigue rapidement progressive, trois une thyroïdite de Hashimoto et un une polyarthrite rhumatoïde ${ }^{111}$. Dans une étude castémoins comparant les AAA classiques versus les anévrysmes inflammatoires, une incidence plus élevée de maladies auto-immunes ou inflammatoires systémiques a été constatée dans le groupe des anévrysmes inflammatoires (6 patients sur 31) ${ }^{112}$. Les autres associations fréquemment rapportées sont celle de la FRPi avec les vascularites systémiques, la spondylarthrite ankylosante et le lupus érythémateux 
systémique ${ }^{22,111,112}$. Dans notre recueil nous comptions un cas de spondylarthrite ankylosante, un cas de thyroïdite, un cas de pseudopolyarthrite rhizomélique et un de psoriasis. Nous avons recensé un cas FRPi chez un patient atteint de pemphigoïde bulleuse, à notre connaissance, aucun cas rapportant cette association n'a été décrit à ce jour. Un des patients du groupe FRPi était atteint de périartérite noueuse (PAN), cette association a déjà été décrite ${ }^{113}$, soulevant à nouveau la question d'une pathogénèse commune.

Sur le plan biologique, il n'y avait dans notre étude quasiment pas de stigmates biologiques d'autoimmunité. Dans une analyse rétrospective menée par Rafiotta et al., 12 patients sur les 50 avaient des anticorps anti-nucléaire à taux significatif. Les auteurs considéraient cette observation comme le reflet d'un fond auto-immun dans la FRPi ${ }^{114}$. De plus, la présence d'anticorps anti-nucléaire au diagnostic serait prédictif $\mathrm{d}^{\prime}$ une taux de rechute plus important ${ }^{104,114}$. La positivité des auto-anticorps était rare dans notre étude, mais ces données manquaient pour beaucoup d'observations. Un taux d'anticorps anti-nucléaire à titre significatif n'était retrouvé que chez 2 patients, et la recherche d'ANCA et d'anticorps anti-thyroïdiens était négative pour l'ensemble des patients chez qui la recherche était effectuée.

\section{FRP secondaires}

Le traitement par radio-chimiothérapie était au sein de notre population significativement plus fréquent dans le groupe FRP secondaire. L'enjeu au diagnostic est donc de différencier l'étiologie secondaire de la forme idiopathique. Si elles sont diagnostiquées et traitées rapidement, les formes idiopathiques et la plupart des autres formes bénignes de FRP ont un bon pronostic. En revanche, la FRP d'origine maligne a un pronostic sombre, principalement greffé à la pathologie sous-jacente, avec une survie à 6 mois variant de 10 à $50 \%$ selon les séries ${ }^{79,115}$. Cette étape diagnostique commence par un interrogatoire minutieux à la recherche des facteurs de risques de FRPi (exposition à l'amiante, tabagisme par exemple) et de rechercher une cause de FRP secondaire : chirurgie abdominale majeure, antécédent de néoplasie, de radiothérapie, prise de médicaments inducteurs.

Dans notre cohorte, 23 patients soit $41 \%$ des cas avaient une fibrose rétropéritonéale secondaire. II n'existe pas à ce jour de données solides sur la proportion de forme idiopathique ou secondaire de FRP. Dans les formes iatrogènes, il est rapporté une régression de la fibrose à l'arrêt du traitement ${ }^{116}$. Malheureusement, cette information était manquante pour le patient de la cohorte traité par dérivé de l'ergot de seigle. Par ailleurs, l'exposition aux médicaments inducteurs de FRP n'a pas été étudiée dans ce travail, nous ne pouvons donc pas estimer l'impact de tels traitements sur la survenue d'une FRP.

Enfin nous avons recensé 5 patients atteints de FRP secondaire à une maladie associée aux IgG4 (parmi 27 patients investigués). L'un d'entre eux, classé en MAG4 « possible » ne présentait que des critères 
cliniques évocateurs avec une anatomopathologie non contributive. Selon les séries, environs $30 \%$ des patients atteints de MAG4 présentent un taux sérique normal $d^{\prime} \lg \mathrm{G}^{33}$ et l'anatomopathologie est souvent mise en défaut pour remplir l'ensemble des critères stricts requis par le consensus japonais de 2011. De nouveau critères ACR/EULAR ont été proposé en 2019. II s'agit de critères de classification, permettant de classer un patient comme atteint de MAG4 en l'absence de documentation histologique ou d'élévation des IgG4 sériques ${ }^{117}$. Dans une étude italienne de 113 patients atteints de péri aortites chroniques, les taux sériques d'IgG4 étaient élevés chez une minorité de patients (24 patients) et ne permettaient pas d'identifier de sous-groupes cliniques ou pronostiques spécifiques. Seule une fréquence plus élevée de lésions extra rétropéritonéales était constatée chez les patients avec taux sérique élevé ${ }^{118}$. II est actuellement établi qu'une élévation sérique d'IgG4 n'est ni un marqueur très sensible, ni spécifique pour le diagnostic de MAG4. L'histologie est importante pour le diagnostic de la MAG4. Ainsi lorsqu'une biopsie est réalisée, il est indispensable de réaliser un Immunomarquage des plasmocytes.

\section{E. Données cliniques, biologiques et histologiques}

Les manifestations cliniques inaugurales étaient peu spécifiques avec des douleurs abdominales et/ou lombaires pour $75 \%$ des patients, légèrement plus fréquentes dans le groupe FRPi $(84,8 \%$ vs $60,9 \%)$. La présence de symptômes systémiques était recensée dans près de la moitié des cas dans les deux groupes. Les autres symptômes observés étaient le résultat de l'obstruction de la fibrose sur les structures adjacentes. Nous avons observé significativement plus de claudication des membres inférieurs dans le groupe $\mathrm{FRPi}$, suggérant soit à nouveau l'incidence plus élevée de la maladie athéromateuse chez ces patients, soit l'atteinte des artères iliaques par la FRP pourrait également expliquer cette symptomatologie. En dehors de symptômes spécifiques d'une étiologie, le tableau clinique initial ne permettait généralement pas de différencier une FRP idiopathique d'une FRP secondaire.

Dans notre cohorte, la majorité des patients avait un syndrome inflammatoire $(89,4 \%)$, sans différence entre les deux groupes. Cette observation est cohérente avec les données de la littérature puisque ce paramètre peut être retrouvé dans près de $80 \%$ des $\operatorname{cas}^{26}$. Ce syndrome inflammatoire peut être une aide pour le suivi de la maladie ${ }^{63}$.

Quand elle était réalisée, la biopsie montrait essentiellement un tissu fibro-inflammatoire non spécifique. Un seul prélèvement permettait de mettre en évidence une prolifération tumorale lymphoïde, chez un patient aux antécédents de lymphome folliculaire. Ainsi, bien qu'un examen histologique approprié permette de confirmer la présence d'une FRP secondaire d'origine maligne, la biopsie ne devrait pas être proposée en première intention chez des patients dont la présentation scannographique est typique et sans 
autre point d'appel clinique ou radiologique. Elle devrait être envisagée chez les patients qui ne présentent pas d'autre site à biopsier ainsi que chez ceux avec une présentation radiologique atypique. En l'absence de consensus, la technique (transpariétale ou chirurgicale) doit être discutée au cas par $\operatorname{cas}^{26}$.

\section{F. Imagerie}

En ce qui concerne l'imagerie, elle joue un rôle clé dans le diagnostic et le suivi de la FRP. L'échographie de l'arbre urinaire peut être réalisée en première intention en présence d'une insuffisance rénale aigue, à la recherche d'une uropathie obstructive. Elle est en revanche peu rentable pour faire le diagnostic de fibrose rétropéritonéale ${ }^{27}$. Ainsi, le diagnostic de FRP repose sur la tomodensitométrie avec injection de produit de contraste ou l'imagerie par résonance magnétique avec injection de gadolinium, qui permettent de définir précisément l'extension de la maladie et l'atteinte urétérale.

A nouveau, l'enjeu reste de différencier une cause idiopathique d'une cause secondaire, notamment maligne. La publication de Mirault et al. s'est intéressée aux caractéristiques IRM permettant de différencier une cause idiopathique d'une cause maligne chez 50 patients ${ }^{80}$. Ainsi, une masse s'étendant du dessus des artères rénales jusqu'au-dessous de la bifurcation aortique était significativement plus fréquente dans le groupe FRP maligne $(p=0,001)$. L'atteinte des voies urinaires ne différait pas selon l'étiologie de la FRP. Enfin, une attraction médiale des uretères était significativement moins fréquente dans la FRP maligne $(p=0,001)$.

Dans notre série, l'apport diagnostique de l'IRM était limité puisqu'il n'a été réalisé que chez 6 patients, son utilisation se discute toutefois en présence d'une insuffisance rénale sévère, contre indiquant I'injection de produits de contrastes iodés. Chez la quasi-totalité des patients (55/56), une TDM était réalisée. Cependant, le nombres de données manquantes était important, liées au caractère rétrospectif de l'étude. Pour beaucoup de patients, les imageries n'étaient pas disponibles et nous nous sommes donc basés sur les comptes rendus d'imagerie disponibles dans les dossiers informatisés des patients ou bien sur les informations fournies dans les comptes rendus d'hospitalisations et de consultations.

Pour la majorité de nos patients, la masse se développait dans la partie sous rénale de l'aorte, atteignant les artères iliaques dans plus de la moitié des cas. Dans l'analyse prospective de Scheel et al., l'étendue de la masse avait été classée selon la localisation anatomique et l'extension aux uretères ou à la veine cave inférieure, proposant une classification radiologique dans le but de standardiser l'analyse scannographique ${ }^{60}$. Nous $n^{\prime}$ avons pas utilisé la classification de Scheel compte tenu de données manquantes trop importantes.

Parmi les 3 patients présentant une atteinte atypique (atteinte de l'aorte thoracique, fibrose étendue de la portion sus rénale à la bifurcation iliaque), 1 patient était atteint de FRP secondaire à une MAG4 possible, les 2 autres étaient atteint d'une fibrose rétropéritonéale idiopathique mais la recherche de MAG4 
n'était pas effectuée. Dans une étude italienne, les auteurs ont montré que chez un tiers des patients, la périaortite chronique concerne également l'aorte thoracique, ce qui suggère à nouveau que ce spectre de maladie représente en fait une maladie inflammatoire primaire et diffuse de l'aorte et de ses branches principales. Devant une telle atteinte, les auteurs suggèrent de rechercher systématiquement une MAG4 ou une maladie d'Erdheim Chester ${ }^{78}$.

L'attraction médiale des uretères était mentionnée pour 7 patients dont 5 étaient atteints de FRPi, 1 de FRP dans un contexte de MAG4 et 1 FRP d'origine maligne. Un refoulement latéral des uretères était précisé pour un patient et concernait une FRPi. II est difficile de conclure compte tenu du faible nombre d'observation dont nous disposons, mais les critères précédemment cités pour différencier une étiologie primitive d'une secondaire peuvent être mis à défaut. Par exemple, une étude américaine suggère que l'absence de déplacement aortique antérieur n'est pas la règle pour tous les patients atteints de FRPi et que certaines FRP malignes présentent cette caractéristique ${ }^{119}$. L'épaisseur de la masse en arrière de l'aorte n'était renseignée que pour 3 de nos patients.

Dans notre population, 37,5\% des patients avait une atteinte urétérale, elle était bilatérale pour un tiers. Dans la cohorte de Scheel et al. $62 \%$ des patients avaient une obstruction d'un ou des deux uretères ${ }^{60}$. Dans l'étude allemande de Brandt et al., une obstruction urétérale est survenue chez 95,6 \% des patients et a entraîné une hydronéphrose bilatérale chez $55,9 \%$ des patients ${ }^{120}$. Ce taux élevé est expliqué par la nature des données qui sont issues d'un registre urologique.

Des adénopathies étaient observées dans $21 \%$ des cas de FRPi et $52 \%$ des cas de FRP secondaires. Dans la série Néerlandaise de FRPi, elles étaient observées dans $25 \%$ des cas $^{57}$. Ces observations peuvent être le témoin du caractère inflammatoire de la fibrose, sans pouvoir conclure sur la différence observée entre nos deux populations.

Le PET TDM peut avoir un rôle dans le diagnostic et dans le suivi de la maladie ${ }^{75,77,121}$. Dans notre étude, un PET TDM a été réalisé pour 26 patients, avec un hyper métabolisme pour 23. Dans une large étude évaluant le rôle pronostique du PET scanner, publiée par Vaglio et al., il a été montré que les patients avec un PET TDM négatif initialement avaient des taux de réponses plus faibles, mais pas absents. En outre, l'intensité de fixation n'était pas corrélée au taux de réponse. Ainsi les auteurs suggèrent tout de même que les patients présentant une maladie active sans hyperfixation au PET TDM doivent être traités. Enfin, la persistance d'une fixation en fin de traitement pourrait également être utile pour détecter une activité métabolique résiduelle et guider le suivi et les choix thérapeutiques ultérieurs ${ }^{122}$. Parmi les 23 patients avec hyperfixation, 12 étaient considérés en rémission à la fin du suivi. La disponibilité du PET TDM peut représenter un frein à son recours. L'intérêt spécifique, et potentiellement plus intéressant, de la TEP-TDM à la recherche d'arguments pour une FRP secondaire n'a pas été évalué à ce jour, bien qu'une absence de fixation rende l'hypothèse d'une cause néoplasique moins probable ${ }^{76}$. 


\section{G. Prise en charge thérapeutique}

La prise en charge urologique visant à lever l'obstruction urétérale et le traitement médical destiné à stopper l'inflammation et réduire la taille de la fibrose sont les piliers de la prise en charge de la FRP. Le délai moyen de prise en charge des patients de la cohorte était de 2,1 mois variant d'une prise en charge immédiate à un délai de 36 mois depuis le diagnostic. Rappelant les dommages irréversibles potentiellement causés par une obstruction urétérale bilatérale et l'absence de réponse aux corticoïdes chez certains patients du fait de la composante fibreuse trop importante, il semble raisonnable de proposer une prise charge la plus rapide possible, qu'elle soit médicamenteuse ou urologique.

L'utilisation des corticoïdes en première intention est désormais la référence. Dans notre série de FRPi, la majorité des patients a été traitée par corticoïdes (26/33 patients). Le schéma thérapeutique ainsi que la durée de la corticothérapie ne sont pas codifiés. Dans un essai contrôlé, après un mois de traitement par prednisone orale à la dose de $1 \mathrm{mg} / \mathrm{kg}$ par jour, les patients étaient randomisés en deux groupes (poursuite de la corticothérapie à dose décroissante sur huit mois versus tamoxifène) avec une supériorité franche de la poursuite de la corticothérapie. Ainsi il semblerait que la dose de $1 \mathrm{mg} / \mathrm{kg} / \mathrm{j}$ pendant 1 mois suivie d'une décroissance progressive pendant 9 à 12 mois soit une stratégie efficace ${ }^{84,89}$. Dans notre étude, l'attitude concernant la corticothérapie était sensiblement superposable a celle proposée par Vaglio et al. La durée moyenne de la corticothérapie était de presque 16 mois.

Vingt des 36 patients $(55,5 \%)$ traités par corticoïdes ont eu des effets indésirables, parmi lesquels diabète cortico-induit et complications infectieuses. La série de Van Bommel rapporte un taux de 33\% de complications sous corticothérapie ${ }^{89}$. Une étude anglaise suggère que certains patients seraient plus susceptibles de bénéficier d'une corticothérapie : patients avec composante inflammatoire sur la biopsie et patients avec fixation intense au PET scanner ${ }^{76}$. Parmi les 23 patients ayant une fixation au PET TDM, seuls 2 n'ont pas été traités, sans complication rapportée dans le suivi.

Près de $60 \%$ des patients $(n=35)$ a nécessité une pose de sonde JJ, dont $28 \%$ bilatérales. Parmi ces patients, 12 n'ont pas eu de traitement médicamenteux associé. La chirurgie, traitement historique de la FRP, est devenue un traitement adjuvant, depuis l'utilisation des corticoïdes. L'urétérolyse, qui peut être proposée lorsque le traitement médical ne permet pas de réduire la masse ou que l'obstruction urétérale persiste, a été chez réalisée chez un seul patient. A la fin du suivi, aucun des patients avec traitement urologique seul n'était considéré en rémission, les sondes double J étaient retirées chez un seul patient, un autre a nécessité une néphrectomie, pour deux autres patients une néphrostomie était réalisée et enfin un patient a bénéficié d'une urétéro-iléoplastie. Concernant la tolérance des sonde JJ, $51 \%$ des patients a présenté des effets indésirables en lien avec ce matériel dont 2 graves (chocs septiques sur pyélonéphrites obstructives). Une équipe française s'est intéressée à la qualité de vie des patients porteurs de sondes JJ ; rapportant une 
morbidité importante avec $56 \%$ d'hématurie, $36 \%$ de dysurie et $78 \%$ d'impériosités mictionnelles et sur une échelle visuelle analogique de $100 \mathrm{~mm}$, la tolérance générale était estimée à $41 \mathrm{~mm}{ }^{123}$. La qualité de vie des patients est d'autant plus impactée par les hospitalisations itératives nécessaires pour le changement régulier de ces sondes. A la fin de période de recueil, 11 patients avaient toujours leur sonde JJ. Cela peut être expliqué par une maladie non contrôlée et la nécessité persistante d'une dérivation urétérale mais également par l'absence de réévaluation de l'indication de ces sondes. Ainsi toutes ces observations confirment que la durée d'implantation doit être aussi courte que possible et qu'une réévaluation régulière de leur indication est nécessaire, notamment par le biais de réévaluations scannographiques régulières.

Dans certains cas, aucun traitement médical n'était instauré ( $n=7 \mathrm{FRPi}$ ). Parmi eux, un seul nécessitait l'introduction d'une corticothérapie par la suite, les autres n'avaient pas de complication particulière. L'absence de symptômes, ainsi que l'absence de complication obstructive ou d'évolution après dérivation des urines pourraient en effet être des arguments pour ne pas instaurer de traitement. Ainsi dans certains cas rares, l'abstention thérapeutique pourrait être proposée sous couvert d'une surveillance clinique, biologique et radiologique rapprochée. II n'existe à ce jour aucune recommandation ni expérience rapportée de cette attitude. De plus, il n'existe pas de donnée sur l'efficacité d'un traitement urologique seul par rapport à un traitement par corticoïdes. II est donc difficile de répondre à la question de savoir si un drainage urétéral seul est acceptable en termes de morbidité et de tolérance, en comparaison à une corticothérapie avec les effets indésirables inhérents à cette thérapeutique. Une prise en charge conjointe entre les différentes spécialités amenées à prendre en charge ces patients semble indispensable.

Le choix des traitements de seconde ligne et leur efficacité dans la FRPi repose principalement sur des rapports de cas et recommandations d'experts. La grande variété des molécules utilisées ainsi que leurs indications ne permettent pas une attitude homogène. De plus, il n'existe pas de donnée comparant directement l'efficacité des immunosuppresseurs associées à des corticoïdes et les corticoïdes seuls. Dans notre série, divers traitements immunosuppresseurs étaient utilisés, reflétant l’hétérogénéité des pratiques dans un même centre et ne permettant pas de conclure sur le bénéfice des différentes stratégies.

\section{H. Évolution et suivi}

Les récidives après plusieurs années sont possibles ${ }^{88,124}$. Dans notre cohorte, 5 patients ont récidivé avec un délai médian de 12 mois. II n'existe pas de marqueur permettant de prédire de façon fiable une récidive ${ }^{63,75}$. Dans une étude récente italienne, le sexe masculin et la présence d'anticorps anti-nucléaire représentaient les principaux facteurs de risques de récidive ${ }^{114}$. Dans une autre série publiée par Van Bommel et al., un traitement prolongé de un an par corticoïdes était associé à un taux élevé de succès initial mais également à un taux élevé de récidive ${ }^{89}$. Ainsi, une surveillance prolongée des patients paraît 
justifiée. De plus, la stratification des patients sur la base du risque de récidive pourrait, d'une part, éviter la possibilité de réactivation de la maladie en cas de traitement sous-dosé et minimiser d'autre part les effets indésirables d'une immunosuppression excessive.

Au cours de la FRPi, le pronostic est avant tout lié à l'insuffisance rénale aiguë et à long terme I'insuffisance rénale chronique. L'insuffisance rénale chronique terminale est néanmoins rare avec un taux inférieur à $5 \%$ dans la plupart des séries ${ }^{54,114}$. Dans notre étude, 18 patients (32\%) ont développé une insuffisance rénale chronique, dont 4 (7\%) une insuffisance rénale terminale. Les facteurs de risque de développer une IRC ont été évalués dans une étude de Gallais et al. qui a identifié 3 items : l'âge au début de la maladie, la présence d'un diabète et le chiffre de créatinine. Dans cette série, l'insuffisance rénale

chronique était observée chez près de $25 \%$ des patients ${ }^{105}$. Au sein de notre cohorte, tous les patients avec IRC avaient des facteurs de risque cardio-vasculaire. L'analyse statistique a montré une association entre HTA et IRC, ce qui n'était pas le cas dans l'étude de Gallais et al.

\section{Limites du travail}

La principale limite du travail est liée au caractère rétrospectif de l'étude, avec un faible nombre de patients inclus et de nombreuses données manquantes. Les résultats obtenus dans les deux populations doivent ainsi être interprétés avec précaution.

Une autre limite est l'absence de relecture de l'imagerie qui aurait pu apporter plus de précision sémioradiologique que le compte rendu, avec notamment la possibilité d'utiliser à postériori la classification de Scheel ou les critères de Mirault appliqués au scanner.

Enfin, le parti pris d'inclure les 3 formes de péri aortites chroniques est discutable, même si nous n'avons pas retrouvé de différence entre les formes avec ou sans dilatation de l'aorte, en dehors de l'obésité plus fréquente dans les formes non anévrysmales. 


\section{Conclusion}

Notre étude décrit 56 patients ayant une fibrose rétropéritonéale dont les caractéristiques sont globalement comparables aux autres séries de la littérature. La corticothérapie concerne $82 \%$ des patients ayant une forme primitive, avec un taux de rechute de 35\%, soulignant la nécessité d'un suivi régulier en cours de traitement, mais également à distance compte tenu du risque de récidive. La diversité des traitements de $2^{\text {ème }}$ intention s'explique par l'absence d'essais cliniques bien conduits qui pourraient permettre d'établir des recommandations avec bon niveau de preuve. D'autres études seront nécessaires pour déterminer les modalités thérapeutiques et de suivi optimales, ainsi que les facteurs prédictifs de la réponse au traitement.

Enfin nous n'avons pas mis en évidence de différence significative au sein du groupe FRPi, selon l'existence ou non d'une ectasie de l'aorte ou d'un anévrysme. Des études épidémiologiques de plus grande ampleur seraient intéressantes pour préciser les différents patterns des 3 entités de la périaortite chronique. 


\section{RÉFÉRENCES BIBLIOGRAPHIQUES}

1. Ormond, J. K. Bilateral Ureteral Obstruction due to Envelopment and Compression by an Inflammatory Retroperitoneal Process. Journal of Urology 59, 1072-1079 (1948).

2. Raper, F. P. IDIOPATHIC RETROPERITONEAL FIBROSIS INVOLVING THE URETERS1. British Journal of Urology 28, 436-446 (1956).

3. Uibu, T. et al. Asbestos exposure as a risk factor for retroperitoneal fibrosis. The Lancet 363, 14221426 (2004).

4. van Bommel, E. F. H., Jansen, I., Hendriksz, T. R. \& Aarnoudse, A. L. H. J. Idiopathic Retroperitoneal Fibrosis: Prospective Evaluation of Incidence and Clinicoradiologic Presentation. Medicine 88, 193-201 (2009).

5. Vaglio, A., Salvarani, C. \& Buzio, C. Retroperitoneal fibrosis. Lancet 367, 241-251 (2006).

6. Miller, O. F., Smith, L. J., Ferrara, E. X., McAleer, I. M. \& Kaplan, G. W. Presentation of idiopathic retroperitoneal fibrosis in the pediatric population. Journal of Pediatric Surgery 38, 1685-1688 (2003).

7. Marvisi, C., Accorsi Buttini, E. \& Vaglio, A. Aortitis and periaortitis: The puzzling spectrum of inflammatory aortic diseases. La Presse Médicale 49, 104018 (2020).

8. Stone, J. R. et al. Consensus statement on surgical pathology of the aorta from the Society for Cardiovascular Pathology and the Association for European Cardiovascular Pathology: I. Inflammatory diseases. Cardiovascular Pathology 24, 267-278 (2015).

9. Vaglio, A. et al. Evidence of autoimmunity in chronic periaortitis: a prospective study. Am J Med $114,454-462$ (2003).

10. Vaglio, A. \& Buzio, C. Chronic periaortitis: a spectrum of diseases: Current Opinion in Rheumatology 17, 34-40 (2005).

11. Pipitone, N., Vaglio, A. \& Salvarani, C. Retroperitoneal fibrosis. Best Practice \& Research Clinical Rheumatology 26, 439-448 (2012).

12. Salvarani, C. et al. Vasculitis of the Gastrointestinal Tract in Chronic Periaortitis. Medicine 90, 28-39 (2011).

13. Goldoni, M. et al. Asbestos and Smoking as Risk Factors for Idiopathic Retroperitoneal Fibrosis: A Case-Control Study. Ann Intern Med 161, 181 (2014).

14. Martorana, D. et al. Chronic periaortitis and HLA-DRB1*03: Another clue to an autoimmune origin. Arthritis Rheum 55, 126-130 (2006).

15. Boiardi et al. - 2011 - CC chemokine receptor 5 polymorphism in chronic pe.pdf. 
16. Ceresini, G. et al. Association between idiopathic retroperitoneal fibrosis and autoimmune thyroiditis: A case-control study. Autoimmunity Reviews 14, 16-22 (2015).

17. Vaglio, A. et al. Peripheral inflammatory arthritis in patients with chronic periaortitis: report of five cases and review of the literature. Rheumatology 47, 315-318 (2007).

18. Afeltra, A. et al. Retroperitoneal Fibrosis and Ankylosing Spondylitis: Which Links? Seminars in Arthritis and Rheumatism 35, 43-48 (2005).

19. Vaglio, A. et al. ANCA-positive periaortic vasculitis: does it fall within the spectrum of vasculitis? J Intern Med 251, 268-271 (2002).

20. Blockmans, D., Baeyens, H., Van Loon, R., Lauwers, G. \& Bobbaers, H. Periaortitis and Aortic Dissection due to Wegener's Granulomatosis. Clinical Rheumatology 19, 161-164 (2000).

21. Fujii, K. \& Hidaka, Y. Churg-Strauss Syndrome Complicated by Chronic Periaortitis: A Case Report and Review of the Literature. Intern. Med. 51, 109-112 (2012).

22. Demko, T. M., Diamond, J. R. \& Groff, J. Obstructive nephropathy as a result of retroperitoneal fibrosis: a review of its pathogenesis and associations. J Am Soc Nephrol 8, 684-688 (1997).

23. Famularo, G. et al. Retroperitoneal fibrosis associated with psoriasis: a case series. Scandinavian Journal of Rheumatology 38, 68-69 (2009).

24. Hennessey, J. V. Riedel's Thyroiditis: A Clinical Review. The Journal of Clinical Endocrinology \& Metabolism 96, 3031-3041 (2011).

25. Rossi, G. M. et al. Idiopathic Mediastinal Fibrosis: a Systemic Immune-Mediated Disorder. A Case Series and a Review of the Literature. Clinic Rev Allerg Immunol 52, 446-459 (2017).

26. Vaglio, A. \& Maritati, F. Idiopathic Retroperitoneal Fibrosis. JASN 27, 1880-1889 (2016).

27. Urban, M. L. et al. Idiopathic and secondary forms of retroperitoneal fibrosis: A diagnostic approach. La Revue de Médecine Interne 36, 15-21 (2015).

28. Tanaka, T. \& Masumori, N. Current approach to diagnosis and management of retroperitoneal fibrosis. Int. J. Urol. 27, 387-394 (2020).

29. Pfitzenmeyer, P. et al. Pleuropulmonary changes induced by ergoline drugs. European Respiratory Journal 9, 1013-1019 (1996).

30. Ahmad, S. Methyldopa and retroperitoneal fibrosis. Am Heart J 105, 1037-1038 (1983).

31. Couderc, M., Mathieu, S., Dubost, J.-J. \& Soubrier, M. Retroperitoneal Fibrosis During Etanercept Therapy for Rheumatoid Arthritis. J Rheumatol 40, 1931-1933 (2013).

32. Khosroshahi, A. et al. Rethinking Ormond's Disease: "Idiopathic" Retroperitoneal Fibrosis in the Era of IgG4-Related Disease. Medicine 92, 82-91 (2013).

33. Stone, J. H. \& Deshpande, V. IgG4-Related Disease. The New England Journal of Medicine 13 (2012). 
34. Schleinitz, N., Briantais, A. \& Ebbo, M. Maladie associée aux IgG4 : des critères « diagnostiques » aux critères de classification ACR/EULAR 2019. La Revue de Médecine Interne 41, 814-821 (2020).

35. Okazaki, K. \& Umehara, H. Are Classification Criteria for IgG4-RD Now Possible? The Concept of IgG4-Related Disease and Proposal of Comprehensive Diagnostic Criteria in Japan. International Journal of Rheumatology 2012, 1-9 (2012).

36. Umehara, H. et al. Comprehensive diagnostic criteria for IgG4-related disease (IgG4-RD), 2011. Mod Rheumatol 22, 21-30 (2012).

37. Shimosegawa, T. et al. International Consensus Diagnostic Criteria for Autoimmune Pancreatitis. 40, 7 (2011).

38. Mizushima, I. et al. Clinical and Pathological Characteristics of IgG4-Related Periaortitis/Periarteritis and Retroperitoneal Fibrosis Diagnosed Based on Experts' Diagnosis. Annals of Vascular Diseases 12, 460 472 (2019).

39. Kasashima, S. et al. A new clinicopathological entity of IgG4-related inflammatory abdominal aortic aneurysm. Journal of Vascular Surgery 49, 1264-1271 (2009).

40. Castelein, T., Coudyzer, W. \& Blockmans, D. IgG4-related periaortitis vs idiopathic periaortitis: is there a role for atherosclerotic plaque in the pathogenesis of IgG4-related periaortitis? Rheumatology 54, 1250-1256 (2015).

41. Khosroshahi, A. et al. Rethinking Ormond's Disease: "Idiopathic" Retroperitoneal Fibrosis in the Era of IgG4-Related Disease. Medicine 92, 82-91 (2013).

42. Koo, B. S. et al. Clinicopathologic characteristics of IgG4-related retroperitoneal fibrosis among patients initially diagnosed as having idiopathic retroperitoneal fibrosis. Modern Rheumatology 25, 194198 (2015).

43. Yamashita, K. et al. Degree of IgG4+ plasma cell infiltration in retroperitoneal fibrosis with or without multifocal fibrosclerosis. Histopathology 52, 404-409 (2008).

44. Zen, Y. et al. Retroperitoneal Fibrosis: A Clinicopathologic Study With Respect to Immunoglobulin G4. American Journal of Surgical Pathology 33, 1833-1839 (2009).

45. Maritati, F. et al. Rituximab therapy for chronic periaortitis. Ann Rheum Dis 71, 1262-1264 (2012).

46. Carruthers, M. N. et al. Rituximab for IgG4-related disease: a prospective, open-label trial. Ann Rheum Dis 74, 1171-1177 (2015).

47. Dedinská, I. et al. Treatment of Ormond Disease and Idiopathic Membranous Glomerulonephritis Using Rituximab. 10, 4 (2016).

48. Ramshaw, A. L., Roskell, D. E. \& Parums, D. V. Cytokine gene expression in aortic adventitial inflammation associated with advanced atherosclerosis (chronic periaortitis). Journal of Clinical Pathology 47, 721-727 (1994). 
49. Vaglio, A. et al. Brief Report: Interleukin-6 as an Inflammatory Mediator and Target of Therapy in Chronic Periaortitis: IL-6 in Chronic Periaortitis. Arthritis \& Rheumatism 65, 2469-2475 (2013).

50. Corradi, D. et al. Idiopathic retroperitoneal fibrosis: Clinicopathologic features and differential diagnosis. Kidney International 72, 742-753 (2007).

51. Zen, Y. et al. Th2 and regulatory immune reactions are increased in immunoglobin G4-related sclerosing pancreatitis and cholangitis. Hepatology 45, 1538-1546 (2007).

52. Kollert, F. et al. CCL18 - Potential Biomarker of Fibroinflammatory Activity in Chronic Periaortitis. J Rheumatol 39, 1407-1412 (2012).

53. Mangieri, D. et al. Eotaxin/CCL11 in idiopathic retroperitoneal fibrosis. Nephrology Dialysis Transplantation 27, 3875-3884 (2012).

54. Kermani, T. A., Crowson, C. S., Achenbach, S. J. \& Luthra, H. S. Idiopathic Retroperitoneal Fibrosis: A Retrospective Review of Clinical Presentation, Treatment, and Outcomes. Mayo Clinic Proceedings 86, 297303 (2011).

55. Scheel et Feeley - 2009 - Retroperitoneal Fibrosis The Clinical, Laboratory.pdf.

56. Kermani et al. - 2011 - Idiopathic Retroperitoneal Fibrosis A Retrospecti.pdf.

57. van Bommel et al. - 2009 - Idiopathic Retroperitoneal Fibrosis Prospective E.pdf.

58. Caiafa, R. O. et al. Retroperitoneal Fibrosis: Role of Imaging in Diagnosis and Follow-up. RadioGraphics 33, 535-552 (2013).

59. Palmisano, A., Cobelli, R., Buzio, C. \& Vaglio, A. Peri-renal Collateral Circles. Urology 74, 292-293 (2009).

60. Scheel, P. J. \& Feeley, N. Retroperitoneal Fibrosis: The Clinical, Laboratory, and Radiographic Presentation. Medicine 88, 202-207 (2009).

61. Williams, R. G. \& Nelson, J. A. Retractile Mesenteritis: Initial Presentation as Colonic Obstruction. Radiology 126, 35-37 (1978).

62. Magrey, M. N., Husni, M. E., Kushner, I. \& Calabrese, L. H. Do acute-phase reactants predict response to glucocorticoid therapy in retroperitoneal fibrosis? Arthritis Rheum 61, 674-679 (2009).

63. Pelkmans, L. G., Aarnoudse, A.-J. L. H., Hendriksz, T. R. \& van Bommel, E. F. H. Value of acute-phase reactants in monitoring disease activity and treatment response in idiopathic retroperitoneal fibrosis. Nephrology Dialysis Transplantation 27, 2819-2825 (2012).

64. Gilkeson, G. S. \& Allen, N. B. A True Connective Tissue Disease. 16.

65. Mitchinson, M. J. The pathology of idiopathic retroperitoneal fibrosis. Journal of Clinical Pathology 23, 681-689 (1970).

66. Mitchinson, M. J. Chronic periaortitis and periarteritis. Histopathology 8, 589-600 (1984). 
67. Mitchinson, M. J. Aortic disease in idiopathic retroperitoneal and mediastinal fibrosis. Journal of Clinical Pathology 25, 287-293 (1972).

68. Wu, J., Catalano, E. \& Coppola, D. Retroperitoneal Fibrosis (Ormond's Disease): Clinical Pathologic Study of Eight Cases. Cancer Control 9, 432-437 (2002).

69. Moul, J. W. Retroperitoneal Fibrosis Following Radiotherapy for Stage I Testicular Seminoma. Journal of Urology 147, 124-126 (1992).

70. Sindelar, W. F., Hoekstra, H., Restrepo, C. \& Kinsella, T. J. Pathological tissue changes following intraoperative radiotherapy. Am J Clin Oncol 9, 504-509 (1986).

71. Haroche, J. et al. Maladie d'Erdheim-Chester. La Revue de Médecine Interne 35, 715-722 (2014).

72. Cronin, C. G. et al. Retroperitoneal Fibrosis: A Review of Clinical Features and Imaging Findings. American Journal of Roentgenology 191, 423-431 (2008).

73. Rubenstein, W. et al. CT of fibrous tissues and tumors with sonographic correlation. American Journal of Roentgenology 147, 1067-1074 (1986).

74. Degesys, G. et al. Retroperitoneal fibrosis: use of CT in distinguishing among possible causes. American Journal of Roentgenology 146, 57-60 (1986).

75. Vaglio, A. et al. 18F-fluorodeoxyglucose positron emission tomography in the diagnosis and followup of idiopathic retroperitoneal fibrosis. Arthritis Rheum 53, 122-125 (2005).

76. Fernando, A. et al. [ 18 F]-Fluorodeoxyglucose Positron Emission Tomography in the Diagnosis, Treatment Stratification, and Monitoring of Patients with Retroperitoneal Fibrosis: A Prospective Clinical Study. European Urology 71, 926-933 (2017).

77. Accorsi Buttini, E., Maritati, F. \& Vaglio, A. [ 18 F]-Fluorodeoxyglucose Positron Emission Tomography and Response to Therapy in Idiopathic Retroperitoneal Fibrosis. European Urology 73, 145146 (2018).

78. Palmisano, A. et al. Chronic periaortitis with thoracic aorta and epiaortic artery involvement: a systemic large vessel vasculitis? Rheumatology 54, 2004-2009 (2015).

79. Miller, R. Malignant Retroperitoneal Differentiation. 172, 5 (1989).

80. Mirault, T. et al. Malignant Retroperitoneal Fibrosis: MRI Characteristics in 50 Patients. Medicine 91, 242-250 (2012).

81. Palmisano, A. \& Vaglio, A. Chronic periaortitis: a fibro-inflammatory disorder. Best Practice \& Research Clinical Rheumatology 23, 339-353 (2009).

82. Gianfreda, D. et al. Erdheim-Chester Disease as a Mimic of IgG4-Related Disease: A Case Report and a Review of a Single-Center Cohort. Medicine 95, e3625 (2016).

83. Ellimoottil, C., Hart, S., Mehta, V. \& Quek, M. L. Localized Perirenal Retroperitoneal Fibrosis. Urology 81, e27-e28 (2013). 
84. Vaglio, A. et al. Prednisone versus tamoxifen in patients with idiopathic retroperitoneal fibrosis: an open-label randomised controlled trial. The Lancet 378, 338-346 (2011).

85. van Bommel, E. F. H., Pelkmans, L. G., van Damme, H. \& Hendriksz, T. R. Long-term safety and efficacy of a tamoxifen-based treatment strategy for idiopathic retroperitoneal fibrosis. European Journal of Internal Medicine 24, 444-450 (2013).

86. Mertens, S., Zeegers, A. G., Wertheimer, P. A., Hendriksz, T. R. \& van Bommel, E. F. Efficacy and complications of urinary drainage procedures in idiopathic retroperitoneal fibrosis complicated by extrinsic ureteral obstruction: Urinary drainage in iRPF. Int J Urol 21, 283-288 (2014).

87. Srinivasan, A. K., Richstone, L., Permpongkosol, S. \& Kavoussi, L. R. Comparison of Laparoscopic With Open Approach for Ureterolysis in Patients With Retroperitoneal Fibrosis. Journal of Urology 179, 1875-1878 (2008).

88. Kardar, A. H., Kattan, S., Lindstedt, E. \& Hanash, K. Steroid Therapy For Idiopathic Retroperitoneal Fibrosis: Dose And Duration. Journal of Urology 168, 550-555 (2002).

89. van Bommel, E. F. H., Siemes, C., Hak, L. E., van der Veer, S. J. \& Hendriksz, T. R. Long-Term Renal and Patient Outcome in Idiopathic Retroperitoneal Fibrosis Treated With Prednisone. American Journal of Kidney Diseases 49, 615-625 (2007).

90. van Bommel, E. F. H., Hendriksz, T. R., Huiskes, A. W. L. C. \& Zeegers, A. G. M. Brief Communication: Tamoxifen Therapy for Nonmalignant Retroperitoneal Fibrosis. Ann Intern Med 144, 101 (2006).

91. Brandt, A. S., Kamper, L., Kukuk, S., Haage, P. \& Roth, S. Tamoxifen Monotherapy in the Treatment of Retroperitoneal Fibrosis. Urol Int 93, 320-325 (2014).

92. Grotz, W., von Zedtwitz, I., Andre, M. \& Schollmeyer, P. Treatment of retroperitoneal fibrosis by mycophenolate mofetil and corticosteroids. The Lancet 352, 1195 (1998).

93. Jois, R. N., Kerrigan, N. \& Scott, D. G. I. Mycophenolate mofetil for maintenance of remission in idiopathic retroperitoneal fibrosis. Rheumatology 46, 717-718 (2006).

94. Khalil, F., Mir, M. A. \& Venuto, R. C. Mycophenolate mofetil in the treatment of retroperitoneal fibrosis. Clin Rheumatol 27, 679-681 (2008).

95. Jr, P. J. S., Feeley, N. \& Sozio, S. M. Combined Prednisone and Mycophenolate Mofetil Treatment for Retroperitoneal Fibrosis. 7.

96. Alberici, F. et al. Methotrexate plus prednisone in patients with relapsing idiopathic retroperitoneal fibrosis. Ann Rheum Dis 72, 1584-1586 (2013).

97. Scavalli, A. S. et al. Long-term follow-up of low-dose methotrexate therapy in one case of idiopathic retroperitoneal fibrosis. Clin Rheumatol 14, 481-484 (1995).

98. Průcha, M., Kolombo, I. \& Štádler, P. Combination of Steroids and Azathioprine in the Treatment of Ormond's Disease - A Single Centre Retrospective Analysis. Prague Med. Rep. 117, 34-41 (2016). 
99. Binder, M. et al. Cyclophosphamide is a highly effective and safe induction therapy in chronic periaortitis: a long-term follow-up of 35 patients with chronic periaortitis. Ann Rheum Dis 71, 311-312 (2012).

100. Scheel, P. J., Piccini, J., Rahman, M. H., Lawler, L. \& Jarrett, T. Combined Prednisone and Mycophenolate Mofetil Treatment for Retroperitoneal Fibrosis. Journal of Urology 178, 140-144 (2007).

101. Marcolongo, R. et al. Immunosuppressive therapy for idiopathic retroperitoneal fibrosis: a retrospective analysis of 26 cases. Am J Med 116, 194-197 (2004).

102. Loricera, J. et al. Tocilizumab in refractory aortitis: a study on 16 patients and literature review. 11.

103. Fry, A. C. et al. Successful Use of Steroids and Ureteric Stents in 24 Patients with Idiopathic Retroperitoneal Fibrosis: A Retrospective Study. Nephron Clin Pract 108, c213-c220 (2008).

104. Moriconi, D. et al. Risk factors for relapse and long-term outcome of idiopathic retroperitoneal fibrosis. Clin Exp Nephrol 23, 1147-1153 (2019).

105. Gallais Sérézal, l. et al. Fibrose rétropéritonéale idiopathique : évolution à long terme du pronostic rénal dans une série rétrospective multicentrique de 30 cas. La Revue de Médecine Interne 35, 570-576 (2014).

106. Maritati, F., lannuzzella, F., Pavia, M. P., Pasquali, S. \& Vaglio, A. Kidney involvement in mediumand large-vessel vasculitis. J Nephrol 29, 495-505 (2016).

107. Lugosi, M. et al. Fibrose(s) rétropéritonéale(s) : stratégie diagnostique, pathologies associées et suivi à long terme d'une cohorte française. La Revue de Médecine Interne 34, 591-599 (2013).

108. Koep, L. \& Zuidema, G. D. The clinical significance of retroperitoneal fibrosis. Surgery $81,250-257$ (1977).

109. Thomas, M. H. \& Chisholm, G. D. Retroperitoneal Fibrosis Associated with Malignant Disease. Br J Cancer 28, 453-458 (1973).

110. Liu, H., Zhang, G., Niu, Y., Jiang, N. \& Xiao, W. Retroperitoneal fibrosis: a clinical and outcome analysis of 58 cases and review of literature. Rheumatol Int 34, 1665-1670 (2014).

111. Vaglio, A. et al. Evidence of autoimmunity in chronic periaortitis: a prospective study. The American Journal of Medicine 114, 454-462 (2003).

112. Haug, E. S. et al. Inflammatory aortic aneurysm is associated with increased incidence of autoimmune disease. Journal of Vascular Surgery 38, 492-497 (2003).

113. van Bommel, E. F. H., Brouwers, A. J. W. B., Makkus, A. C. F. \& van Vliet, A. C. M. Retroperitoneal fibrosis and p-ANCA-associated polyarteritis nodosa: coincidental or common etiology? European Journal of Internal Medicine 13, 392-395 (2002).

114. Raffiotta, F. et al. Idiopathic Retroperitoneal Fibrosis: Long-term Risk and Predictors of Relapse. American Journal of Kidney Diseases 74, 742-750 (2019).

115. CHISHOLCMh, G. D. MALIGNANT OBSTRUCTIVE UR\&MIA. BRITISH JOURNAL OF UROLOGY 7. 
116. Graham, J. R., Suby, H. I., LeCompte, P. R. \& Sadowsky, N. L. Fibrotic disorders associated with methysergide therapy for headache. N Engl J Med 274, 359-368 (1966).

117. Wallace, Z. S. et al. The 2019 American College of Rheumatology/European League Against Rheumatism Classification Criteria for IgG4-Related Disease. Arthritis Rheumatol 72, 7-19 (2020).

118. Maritati, F. et al. Clinical and Prognostic Significance of Serum IgG4 in Chronic Periaortitis. An Analysis of 113 Patients. Front. Immunol. 10, 693 (2019).

119. Cohan, R. H. Imaging appearance of fibrosing diseases of the retroperitoneum: can a definitive diagnosis be made? 11.

120. Brandt, A. S., Kamper, L., Kukuk, S., Haage, P. \& Roth, S. Associated Findings and Complications of Retroperitoneal Fibrosis in 204 Patients: Results of a Urological Registry. Journal of Urology 185, 526-531 (2011).

121. Jansen, I., Hendriksz, T. R., Han, S. H., Huiskes, A. W. L. C. \& van Bommel, E. F. H. 18Ffluorodeoxyglucose position emission tomography (FDG-PET) for monitoring disease activity and treatment response in idiopathic retroperitoneal fibrosis. European Journal of Internal Medicine 21, 216-221 (2010).

122. Vaglio, A. et al. Post-treatment residual tissue in idiopathic retroperitoneal fibrosis: active residual disease or silent 'scar' ? A study using 18F-fluorodeoxyglucose positron emission tomography. Clin Exp Rheumatol 23, 231-234 (2005).

123. Chambade, D. et al. [Study of the safety of double J ureteric stents]. Prog Urol 16, 445-449 (2006).

124. Moroni, G. et al. Long-term outcome of idiopathic retroperitoneal fibrosis treated with surgical and/or medical approaches. Nephrology Dialysis Transplantation 21, 2485-2490 (2006). 


\section{RÉSUMÉ}

Introduction : La fibrose rétropéritonéale (FRP) est une maladie rare caractérisée par la présence d'un tissu fibro-inflammatoire engainant les structures rétropéritonéales. Elle peut être idiopathique ou secondaire à diverses étiologies qui doivent être exclues. Le pronostic de la FRPi est lié au risque de survenue d'une insuffisance rénale chronique. Notre objectif était de rapporter les données épidémiologiques de la cohorte rouennaise, et d'analyser les stratégies diagnostiques et thérapeutiques, le suivi et le pronostic chez ces patients.

Patients et méthode : Il s'agissait d'une étude rétrospective monocentrique menée au CHU de Rouen. Les cas de FRP pris en charge dans les services de médecine interne, néphrologie et urologie entre 2010 et 2021 ont été colligés. Le diagnostic de FRP reposait essentiellement sur l'imagerie. Les données épidémiologiques, clinico-biologiques, radiologiques, thérapeutiques et de suivi ont été recueillies de manière rétrospective. Une comparaison a été réalisée entre les formes idiopathique et secondaire.

Résultats : 56 patients ont été inclus avec un âge moyen au diagnostic de $62,8 \pm 12,6$ ans, et un ratio hommes/femmes de 2,7. La FRP était idiopathique pour 33 patients (59\%). Cinq FRP secondaires étaient liées à une MAG4 (22\%) et 8 secondaires (14\%) à une néoplasie. Une preuve histologique était disponible pour 9 patients dont 8 ayant montré un tissu fibro-inflammatoire non spécifique. Les symptômes inauguraux les plus fréquents étaient des douleurs lombaires et/ou abdominales (75\%) et des symptômes systémiques (48\%). Un syndrome inflammatoire biologique était souvent observé (89\%). Le taux de créatinine moyen au diagnostic était de 189,5 mol/L. Dans la population globale, 35 patients (63\%) ont reçu un traitement par corticoïdes ( $82 \%$ dans le groupe idiopathique vs $40 \%$ dans le groupe secondaire, $p=0.002$ ). Le recours à la pose d'une sonde double J a été nécessaire pour 35 patients (63\%), bilatérale pour 16 patients, sans différence entre les 2 groupes. Une rechute et une récidive ont été observées chez 30\% et 16\% respectivement des patients traités par corticoïdes, sans différence significative entre les groupes idiopathique et secondaire. Au terme du suivi, 26 patients (47\%) étaient en rémission (67\% vs 18\%, p=0.001 entre les formes idiopathique et secondaire, respectivement). Un retrait des sondes double J a pu être réalisé chez 14 patients (40\%), principalement chez les patients du groupe idiopathique (35\% vs $13 \%, p=0,11)$. Une insuffisance rénale chronique était notée chez 32\% des patients, et $7 \%$ avec une atteinte terminale.

Conclusion : Les conduites diagnostiques et thérapeutiques des FRP sont hétérogènes et nécessitent une prise en charge pluridisciplinaire. Les formes secondaires ont une évolution moins favorable. Les patients atteints de FRPi justifient un suivi à long terme du fait de récidive possibles et du risque d'insuffisance rénale chronique.

Mots clés : Fibrose rétropéritonéale, péri-aortite chronique, maladie associée aux IgG4 